\title{
Kenya: 2004 Article IV Consultation, First Review Under the Poverty Reduction and Growth Facility, and Requests for Augmentation of Access, Rephasing of the Arrangement, and Waiver of Performance Criteria-Staff Report; Staff Statement; Public Information Notice and Press Release on the Executive Board Discussion; and Statement by the Executive Director for Kenya
}

Under Article IV of the IMF's Articles of Agreement, the IMF holds bilateral discussions with members, usually every year. In the context of a combined discussion of the 2004 Article IV consultation and the first review under the Poverty Reduction and Growth Facility, requests for augmentation of access, rephasing of the arrangement, and waiver of performance criteria for Kenya, the following documents have been released and are included in this package:

- $\quad$ The staff report for the combined 2004 Article IV consultation, First Review Under the Poverty Reduction and Growth Facility, Requests for Augmentation of Access, Rephasing of the Arrangement, and Waiver of Performance Criteria, prepared by a staff team of the IMF, following discussions that ended on September 24, 2004, with the officials of Kenya on economic developments and policies. Based on information available at the time of these discussions, the staff report was completed on December 6, 2004. The views expressed in the staff report are those of the staff team and do not necessarily reflect the views of the Executive Board of the IMF.

- A staff statement of December 20, 2004, updating information on recent developments

- $\quad$ The Public Information Notice (PIN) and Press Release, summarizing the views of the Executive Board as expressed during its December 20, 2004, discussion of the staff report on issues related to the Article IV consultation and the IMF arrangement, respectively.

- $\quad$ A statement by the Executive Director for Kenya.

The documents listed below have been or will be separately released:

Letter of Intent sent to the IMF by the authorities of Kenya* Memorandum of Economic and Financial Policies by the authorities of Kenya* Technical Memorandum of Understanding*

*Also included in the Staff Report

The policy of publication of staff reports and other documents allows for the deletion of market-sensitive information.

Copies of this report are available to the public from

International Monetary Fund $\bullet$ Publication Services

$70019^{\text {th }}$ Street, N.W. • Washington, D.C. 20431

Telephone: (202) 623-7430 • Telefax: (202) 623-7201

E-mail: publications@imf.org •Internet: http://www.imf.org

\section{International Monetary Fund}

Washington, D.C. 

INTERNATIONAL MONETARY FUND

\title{
KENYA
}

\section{Staff Report for the 2004 Article IV Consultation, First Review Under the Poverty Reduction and Growth Facility, and Requests for Augmentation of Access, Rephasing of the Arrangement, and Waiver of Performance Criteria}

\author{
Prepared by the African Department \\ (In consultation with other Departments)
}

Approved by Thomas Krueger and Anthony R. Boote

December 6, 2004

- $\quad$ Two missions visited Nairobi during May 13-June 2 and September 12-24, 2004. The staff team comprised Messrs. Kalinga (head), McIntyre, Cheng (all AFR), Ms. Aylward (PDR), Ms. Brenner, Mr. Podpiera (MFD), and Mr. Tchaidze (FAD). Mr. Reitmaier, the Senior Resident Representative in Nairobi, also participated in the discussions. The staff team met with the Minister of Finance, the Governor of the Central Bank of Kenya, the Permanent Secretary for Finance, other senior officials, and members of the Finance Committee of Parliament, the diplomatic community, and civil society and trade union representatives.

- Kenya has accepted the obligations of Article VIII and maintains an exchange rate system that is free of restrictions on current transactions.

- At the conclusion of the last Article IV consultation on May 2, 2003, Directors commended Kenya for maintaining a measure of financial stability during recent years in difficult circumstances and for the progress made in the governance area. Directors emphasized that key priorities for the PRGFsupported program should include actions to continue to improve governance and fiscal transparency; fiscal consolidation to address the domestic debt problem, and ensure fiscal sustainability over the medium term; a credible strategy to reform tax administration and enhance revenues; measures to restructure public outlays in favor of poverty-related spending and improve the delivery of basic social spending; a clear strategy for restructuring and strengthening the banking system; and privatization of public banks and other public enterprises.

- $\quad$ Conclusion of the first review was delayed because the development of the new wage setting mechanism for public employees took longer than anticipated. Agreement on a fiscal framework for 2004/05 (July/June) was reached in September 2004. The authorities request five waivers for nonobservance of five performance clauses (Attachment).

- The authorities have communicated that they will publish the staff report.

- $\quad$ Kenya's relations with the Fund, including recent technical assistance, are summarized in Appendix I and its relations with the World Bank Group in Appendix II. Statistical issues are discussed in Appendix III. Appendix IV updates the debt sustainability analysis. Appendix V summarizes information on the Millennium Development Goals, and Appendix VI presents data on selected social indicators. Attachment I is the letter from the Minister of Finance of Kenya, with the Memorandum of Economic and Financial Policies (MEFP) and the Technical Memorandum of Understanding (TMU) in the Annex. 


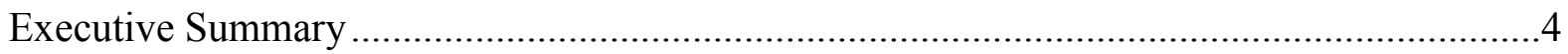

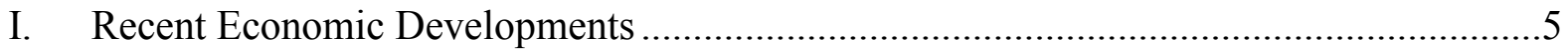

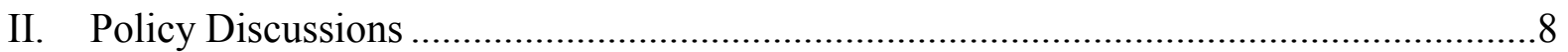

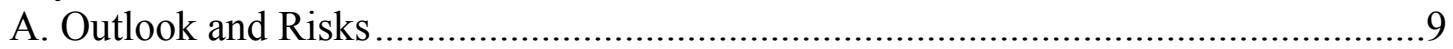

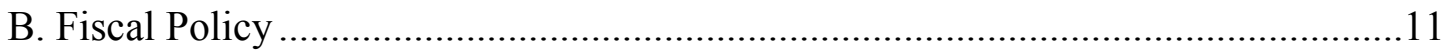

C. Monetary and Exchange Rate Policies...................................................................13

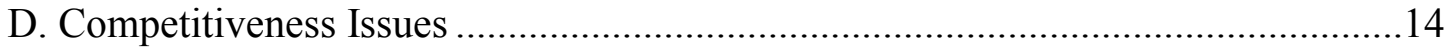

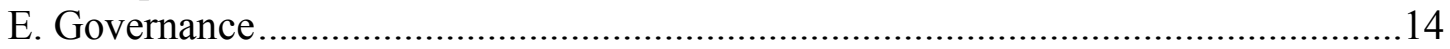

F. Public Enterprise Restructuring and Privatization.................................................17

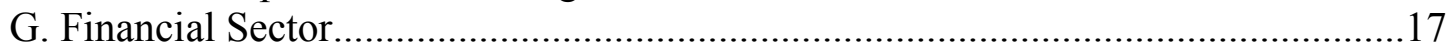

H. External Trade and Regional Integration ............................................................19

I. External Financing Requirements ....................................................................20

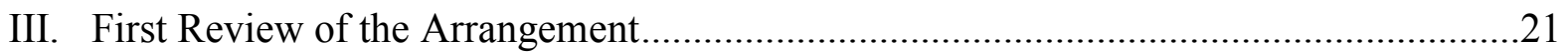

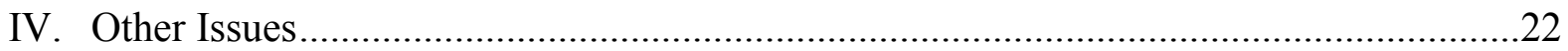

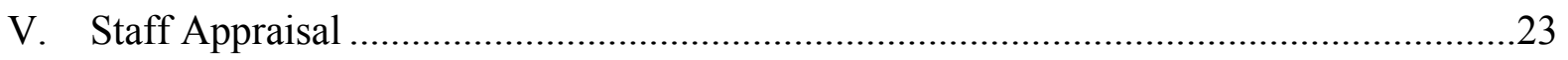

Text Tables

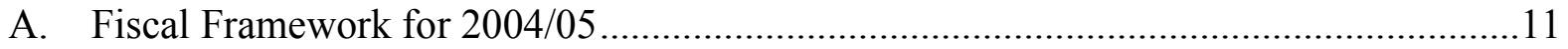

B. Comparative Indicators of the Cost of Doing Business in Kenya ..................................16

C. Comparative Tax Rates in the East African Region ......................................................20

Boxes

1. Past Fund Policy Recommendation and Implementation ..............................................5

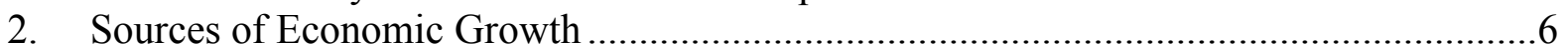

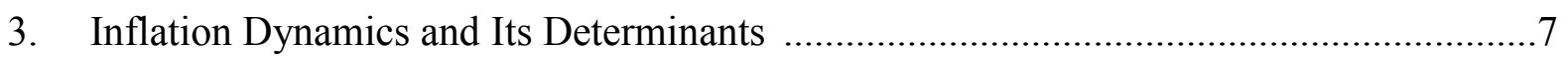

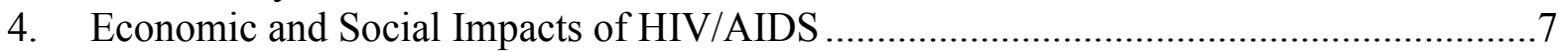

5. Fiscal Strategy Underpinning the PRGF Arrangement ..................................................12

6. Main Conclusions of the Financial Sector Assessment Program .....................................18

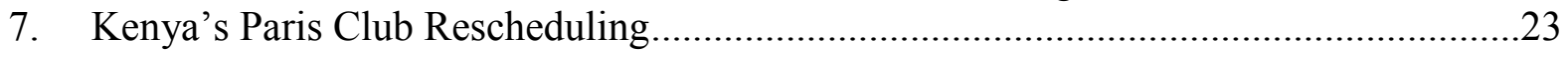

Figures

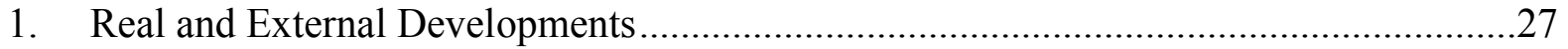

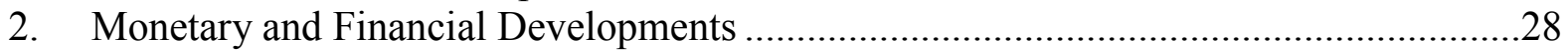

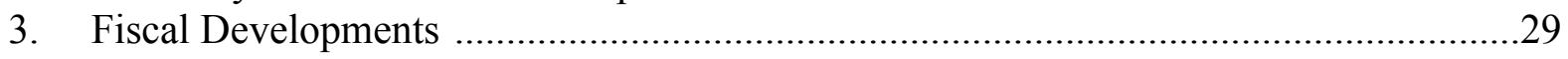




\section{Tables}

1. Medium-Term Macroeconomic Framework, Base Case Scenario, 2002/03-07/08 ........30

2. Monetary Survey, Base Case Scenario, 2003-2007 ….............................................31

3a. Central Government Financial Operations, 2001/02-2007/08 ...................................32

3b. Central Government Financial Operations, 2001/02-2007/08 ....................................33

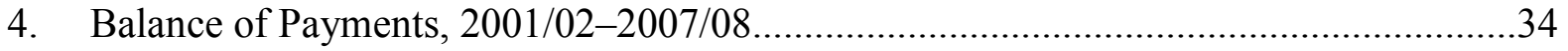

5. Financial Soundness Indicators for the Banking Sector, 1999-2004 ............................35

6. Disbursement Schedule Under the Three-Year PRGF Arrangement ..............................36

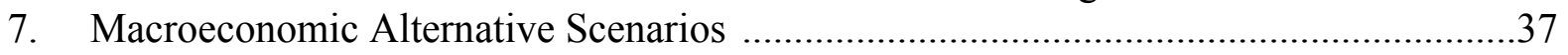

8. External Financing Requirements and Resources, 2001/02-2007/08 ..........................38

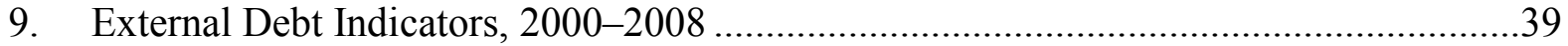

10. Actual and Projected Payments to the Fund, 2001-08 ............................................40

Attachment

Letter of Intent

Annexes

1. Memorandum of Economic and Financial Policies for $2004 / 05$ Under the

PRGF Arrangement ......................................................................... 43

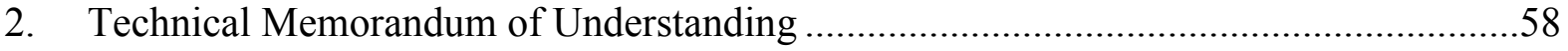

Appendices

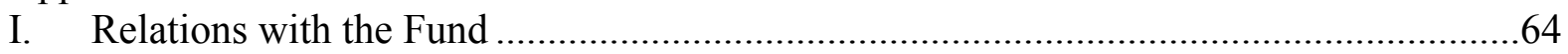

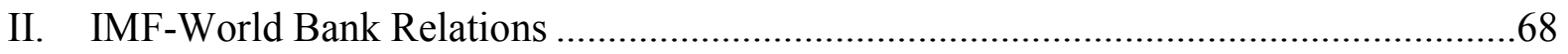

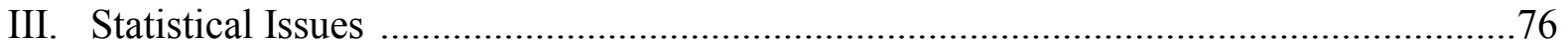

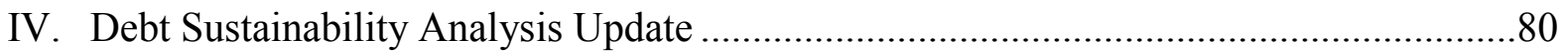

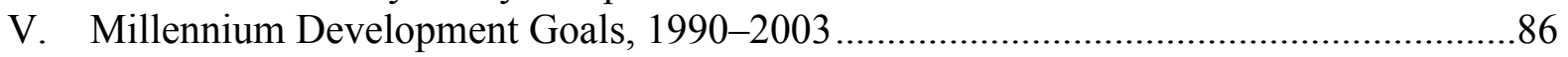

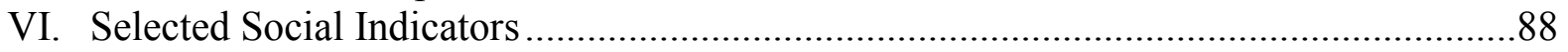




\section{EXECUTIVE SUMMARY}

\section{Political and economic setting}

- As Kenya proceeds into its second year of reforms under the PRGF arrangement, notable progress has been made in implementing the program. While five performance criteria were not met, the new government that took office in December 2002 has begun to address issues that have been, for some time, at the center of Fund policy advice (Box 1).

- $\quad$ Program execution has been adversely affected by the protracted and contentious deliberations on constitutional reform and by capacity constraints in key ministries.

- $\quad$ Some macroeconomic gains were made in 2003/04 (July/June), notably a moderate rebound in economic activity and the drop in domestic debt (in percent of GDP). However, inflation has risen markedly.

- $\quad$ The medium-term outlook is promising if structural reforms are deepened, capacity constraints are addressed, and strong donor support is restored.

- However, there are risks to the macroeconomic outlook. An intensification of political tensions or delays in concluding the constitution review process would raise uncertainty, postpone the resumption of strong budgetary support, and complicate macroeconomic management.

- The authorities are requesting an SDR 50 million (18.4 percent of quota) augmentation of the access under the PRGF arrangement to help address balance of payment pressures resulting from the oil price increase and drought-related food imports.

\section{Policy issues}

Kenya faces several policy challenges:

- Entrenching fiscal discipline. A significant reduction of domestic borrowing is essential for fiscal sustainability.

- $\quad$ Reorienting the budget toward pro-poor spending and improving expenditure management. Putting the economy on a sustainable path of rapid and poverty-reducing growth will require a reorientation of expenditure priorities and modern public expenditure management.

- $\quad$ Restoring price stability. There is a need to better understand the monetary transmission mechanism, to provide a clear commitment to price stability as the main focus of monetary policy, and to use monetary instruments efficiently.

- $\quad$ Enhancing the effectiveness of key governance institutions. Improving capacities and budgetary resources top the agenda.

- $\quad$ Strengthening Kenya's external competitiveness. Reforming labor markets, upgrading infrastructure, and deepening the structural reform agenda are key.

Some progress has been made in these areas and agreement has been reached on the way ahead. The staff recommends completion of the first review and augmentation of access. 


\section{Box 1. Kenya: Past Fund Policy Recommendations and Implementation}

Before 2003, pervasive governance problems and weak political commitment to economic reforms did not provide a favorable environment for the implementation of Fund policy advice. Despite the challenges detailed in this report, implementation of Kenya's economic policies has improved substantially since 2003, when the coalition government led by President Kibaki took office. This box considers recent experience with the implementation of Fund policy in four areas: governance, fiscal sustainability, financial sector, and poverty reduction.

Governance: The Fund has emphasized governance as key to improving Kenya's economic performance; failure to implement reforms in this area led to the interruption of several previous Fund programs. Since December 2002, Kenya has implemented an ambitious and multifaceted program of reforms, including passing key anticorruption and public ethics legislation, strengthening the judiciary, and reforming the policy, as well as the public audit system. Additional steps to strengthen governance are discussed in this report.

Fiscal sustainability: The Fund has urged Kenya to address fiscal sustainability by, among other steps, strengthening revenue performance, lowering the wage bill as a proportion of revenue, and decisively tackling contingent fiscal liabilities. Noting the concern about fiscal sustainability, the government has already taken various measures to this end, including strengthening tax administration, initiating public expenditure management (PEM) reforms, continuing civil service rationalization, and establishing a new wage-setting mechanism for public employees. The government has also stressed the need to increase poverty reduction spending. For this reason, the reorientation of public expenditure in favor of essential social and economic outlays is a key priority of the government

Financial sector: Over the recent past, Kenya has taken important steps to strengthen financial sector supervision in response to Fund advice. It has been less successful in responding to the recommendations of the Fund and the Bank to reduce public sector ownership of banks. The government has indicated that the slow progress in this area reflects primarily fears that privatization may adversely affect financial intermediation in rural areas.

Poverty Reduction: Kenya has produced an ambitious poverty reduction strategy paper. Fund policy advice has emphasized the links from improved PEM, fiscal sustainability, and financial sector stability to enhanced poverty reduction strategies and pro-poor spending. The government has made strides in these areas by addressing budget rigidity through the new wage-setting mechanism for public employees, but it has also indicated that its intentions to restructure expenditure toward poverty reduction have been hampered by uncertain donor support.

\section{RECENT ECONOMIC DEVELOPMENTS}

1. A narrowly based rebound in economic activity has begun. Although real GDP growth at an estimated 2.1 percent in 2003/04 was above the program assumption of 1.9 percent, growth has depended primarily on a recovery of the tertiary sectors (Figure 1). Primary sector output has been adversely affected by a drought, and gross investment (in percent of GDP) has remained at prereform levels (Table 1). Kenya's growth has been driven primarily by factor accumulation, with efficiency gains, as measured by total factor productivity, declining (Box 2). 


\section{The loosening of monetary policy has led to an increase in inflation and a} depreciation of the shilling. In response to the lowering of banks' required reserves from 10 percent to 6 percent in July 2003, the money multiplier rose precipitously, resulting in a 13 percent expansion of broad money in 2003/04 and negative real interest rates (Table 2). Headline inflation rose to 19 percent in September 2004 from 8 percent a year earlier (Figure 1). Kenya's inflation has also been driven by increases in energy prices, and a weakening of the Kenyan shilling (Box 3).

3. Recent economic developments may have worsened poverty indicators. With real GDP growth broadly in line with the increase in population and the economy suffering from the effects of major negative shocks - the drought and the oil price increase - the poverty rate may have increased in the recent past. Moreover, progress on poverty alleviation has been hampered by delays in initiating major poverty reduction programs in the social and economic sectors. AIDS remains a major challenge (Box 4).

\section{Box 2. Kenya: Sources of Economic Growth}

Kenya's economic performance since 1990 has been lackluster, with growth averaging 1.6 percent a year. A simple growth accounting exercise has shown that, like most sub-Saharan African countries, much of the economic growth in Kenya has been driven by factor accumulation, notably physical capital formation and employment growth. Total factor productivity (TFP) broadly declined during the past two decades. However, TFP growth is projected to be positive in 2004. A simple econometric study shows that TFP growth is strongly and positively associated with governance and significantly and negatively associated with inflation. ${ }^{1}$

\begin{tabular}{|c|c|c|c|c|c|}
\hline \multirow[b]{3}{*}{ Period } & \multirow{3}{*}{$\begin{array}{c}\text { Annual } \\
\text { Average } \\
\text { Growth Rate } \\
\text { of Output }\end{array}$} & \multicolumn{4}{|c|}{ Annual Average Contributions to Output Growth } \\
\hline & & \multirow[b]{2}{*}{$\begin{array}{l}\text { Physical } \\
\text { Capital }\end{array}$} & \multicolumn{2}{|c|}{ Human Capital } & \multirow[b]{2}{*}{ TFP } \\
\hline & & & Employment & Education & \\
\hline $1980-84$ & 3.05 & 3.20 & 1.63 & -0.68 & -1.10 \\
\hline $1985-89$ & 5.99 & 2.33 & 2.34 & 0.63 & 0.70 \\
\hline 1990-94 & 0.90 & 1.15 & 1.00 & 0.57 & -1.82 \\
\hline 1995-99 & 2.28 & 1.52 & 1.23 & 0.45 & -0.92 \\
\hline $2000-04$ & 1.56 & 0.84 & 0.53 & 0.45 & -0.27 \\
\hline \multicolumn{6}{|l|}{ Of which: } \\
\hline 2002 & 1.07 & 0.94 & 0.81 & 0.45 & -1.13 \\
\hline 2003 & 1.66 & 0.71 & 0.98 & 0.45 & -0.49 \\
\hline Proj. 2004 & 2.37 & 0.75 & 0.98 & 0.45 & 0.18 \\
\hline
\end{tabular}

${ }^{1}$ For details, see Chapter I of the Selected Issues Paper. 


\section{Box 3. Kenya: Inflation Dynamics and Its Determinants}

Kenya's inflation has recently risen sharply. Annual overall inflation was 19 percent in September 2004, with underlying inflation (overall inflation excluding food, fuel, and energy) reaching 7 percent. These rates have exceeded the Central Bank of Kenya (CBK)'s target of 5 percent for underlying inflation.

Potential factors that may have contributed to Kenya's recent high inflation include an expansionary monetary policy and, on the supply side, a poor harvest and high energy prices. A simple econometric study shows that the recent high overall inflation has been driven primarily by a high growth rate of broad money, a weakening of Kenya's nominal exchange rate, high energy prices, and low agricultural output. ${ }^{1}$

Source: Fund staff.

${ }^{1}$ For a detailed discussion of the econometric model, see Chapter II in the Selected Issues paper.

\section{Box 4. Kenya: Economic and Social Impact of HIV/AIDS}

HIV/AIDS is a serious problem in Kenya, with the infection rate estimated at 14 percent of the adult population. The number of AIDS-related deaths is estimated at 150,000 in 2002.

\section{Economic consequences}

- $\quad$ The high death rates among the working-age population imply that AIDS deaths may directly reduce potential annual economic growth by 1.0-1.2 percentage points a year.

- $\quad$ Poor health of workers weakens labor productivity.

- $\quad$ High medical costs take resources away from other, more economically productive areas.

\section{AIDS and poverty}

- $\quad$ Family income falls when adults fall victim to AIDS or when they reduce work hours to care for sick family members.

- $\quad$ High medical costs push the infected and their families into poverty.

4. Overall fiscal performance strengthened during the fiscal year 2003/04.Central government fiscal imbalances were much lower than expected under the program, leading to a marked drop in the ratio of domestic debt to GDP from 24.3 percent at end-June 2003 to 22.2 percent at end-June 2004 (Table 3 and Figure 3). While this performance reflected the 
positive effects of strong revenue collection and the steps taken to tighten the management of recurrent expenditure, fears that the planned large domestic borrowing might crowd out private activity also induced the authorities to curtail some spending programs. In addition, there were delays in implementing some programs, such as the recapitalization of the National Bank of Kenya.

5. The current account deficit (excluding official transfers), at 2.0 percent of GDP in 2003/04, was narrower than projected (Table 4). The expected strong growth in investment-related imports that was conditioned on strong economic reforms and positive private sector sentiment did not fully materialize. Higher oil (US\$1,048 million) and droughtrelated food imports (US\$83 million) partially offset the lower-than-expected investmentrelated imports. Export volumes grew by 7 percent in 2003/04, as the tea, horticultural, and African Growth and Opportunity Act (AGOA)-related garment sectors performed well. In the face of the smaller current account gap, foreign exchange reserves exceeded the program target.

6. Financial sector performance has improved but major concerns remain. The ratio of nonperforming loans (NPLs) to total loans fell and but decisive action is needed to address the problems of distressed state banks to tackle the NPL problem effectively. Key macroprudential indicators are presented in Table 5.

\section{Implementation of structural reforms has been mixed:}

- The liberalization of the telecommunications sector is on course, with the licensing of a third mobile operator.

- $\quad$ Although in the past years little progress was made in implementing fundamental changes in Kenya Revenue Authority (KRA) operations, significant steps have recently been taken to improve domestic tax administration consistent with previous FAD recommendations.

- The restructuring of expenditures to social sectors, notably education, commenced.

- $\quad$ As outlined in the MEFP (para. 17), promising initial steps have been made in moving toward a modern PEM system.

\section{Policy Discussions}

8. Against the backdrop of a challenging political and economic environment, the discussions focused on the policy requirements for restoring strong economic growth over the medium term. There was agreement on an urgent need for an acceleration of structural reforms, as well as on how Kenya should respond to the slower resumption of donor assistance now foreseen and the risks to the program. For the near term, the discussions concentrated on the appropriate response to the significant shortfalls in donor budgetary support, the reemergence of inflation, and the need for an augmentation of access under the PRGF arrangement of SDR 50 million to ameliorate, in part, the adverse impact of oil prices and the drought on the balance of payments. For the first review of the PRGF arrangement, understandings were reached on performance criteria for end-March 2005 and 
indicative targets for end-September 2005 that are presented in the attached MEFP, and a rephasing of disbursements under the PRGF arrangement (Table 6).

\section{A. Outlook and Risks}

9. The authorities explained that the economic recovery was projected to continue in 2004/05, with real GDP expected to grow by 2.7 percent, because the tourism and construction sectors were expected to perform strongly. However, they pointed out that growth would fall below the original program forecast of 3.1 percent, reflecting in part a significant cut in the projected level of investment. The authorities expected a decline in inflation, excluding food and oil, to 3.5 percent by June 2005 in response to the tightening of monetary policy under the program.

10. Kenya's medium-term prospects depend critically on the pace of reforms, fiscal consolidation, and the resumption of strong donor support. The baseline scenario has growth reaching 4.0 percent in 2007/08 - a rate the staff believes is achievable with a modest reform effort. This scenario, however, assumes much lower amounts of donor assistance than envisaged in the original program because governance concerns have negatively affected donor support to Kenya. For the same reason, the overall deficits (before grants) and the domestic financing requirements, over the medium term, would be higher than envisaged earlier, and the targets for domestic debt are above earlier projections. Nevertheless, the authorities were concerned that although the medium-term growth rates appeared reasonable when seen against Kenya's recent performance, they fall far short of the Economic Recovery Strategy (ERS) target of 6 percent, which implies a significant delay in the achievement of the Millennium Development Goals (MDGs) (Appendix V).

11. The baseline scenario would leave the fiscal position in a fragile position. The authorities indicated that several factors accounted for this. First, the realization of the fiscal objectives depended upon the restoration of strong donor support; sudden slowdowns or stops in such assistance would greatly complicate the attainment of overall fiscal objectives. Second, enhancing fiscal flexibility requires addressing the problems that gave rise to the large budget transfers, which would take time to complete. Third, the coming into force of a new constitution could necessitate a reexamination of the entire medium-term fiscal strategy if new fiscal mandates are created. Fourth, most fiscal variables were procyclical and volatile.

\section{The mission also discussed alternative medium-term scenarios on the downside}

(Table 7). If political tensions increased and the reform momentum slowed significantly, donor budgetary support would likely be suspended and domestic financing would rise substantially. In this scenario, government domestic debt would exceed 25 percent of GDP, economic growth would stall, and there would be little progress on poverty reduction. Government representatives observed that the intention was to avoid this outcome. They pointed out that the commitment of the cabinet to the economic reforms under the program was strong and that the emerging consensus on constitutional reform boded well for the sustained implementation of the program. In contrast, an alternative high-case scenario (Table 7) shows that strong fiscal consolidation and accelerated structural reforms that are 
supported by strong donor support could trigger a virtuous cycle of higher growth and improved public finances.

\section{Notwithstanding projected strong export performance, the external current} account deficit, excluding grants, is expected to widen considerably in 2004/05. This would reflect primarily a drop in gross national savings because the increase in oil and food prices would lower the proportion of income saved. Over the medium term, the current account deficit is forecast to widen through 2005/06 as imports remain robust in response to a pickup in growth and private-sector-led capital spending, but would begin to decline thereafter in response to a strengthening of the national savings effort. Exports are also projected to perform strongly as economic reforms begin to enhance Kenya's competitiveness.

14. The November $\mathbf{2 0 0 3}$ staff report projected large financing gaps to be filled by donor support. These gaps reflected large public investment projects and a buildup of international reserves to lower the economy's external vulnerabilities. The authorities observed that as donor disbursements had been smaller and slower than expected, the current base case scenario projects external financing requirements to average about US\$1.3 billion a year over 2004/05 to 2006/07, well below earlier projections. They expected these requirements to be filled by a combination of donor budgetary support, project support, private financing, debt service relief from the January 2004 Paris Club rescheduling, and Fund disbursements.

\section{Kenya has requested an augmentation of the PRGF arrangement to help} mitigate the adverse effects of the world oil price increase and the drought on the balance of payments. The sharp rise in oil prices and drought-related food imports is projected to raise total imports by up to US\$500 million (3.0 percent of GDP). In response to these developments, the authorities have allowed oil and food prices to adjust fully to the increase in import prices, but with appropriate safety nets to protect the poor. Moreover, several donors, including the World Bank, AfDB, and the European Union, have been approached for financial support to help offset the higher external payments now foreseen. The authorities have also requested an SDR 50 million augmentation of the access under the PRGF arrangement, with SDR 25 million to be disbursed at the completion of the first review and the remaining SDR 25 million at the completion of the second review. The augmentation would bring total access under the program to SDR 225 million or 82.9 percent of quota. While this access is higher than the norm of access for fourth-time users of the PRGF of 45 percent of quota, it is well below the maximum limit for access under the PRGF arrangement of 140 percent. The amount of augmentation does not materially worsen Kenya's debt profile or its ability to repay the Fund.

\section{In discussions of Kenya's vulnerability to external crisis, the staff noted that} Kenya's import cover, while adequate, had declined from 3.5 months in 2001/02 to 3.0 months in 2003/04. It pointed out that a combination of weak growth, large fiscal imbalances and financial sector weakness in an environment of relatively free capital movements, could set the stage for a buildup of vulnerabilities that could eventually become severe in the event of exogenous shocks. The authorities explained that concerns about the fall in foreign reserve cover, when set against the intended lowering of external tariffs from 
January 2005 and the uncertain prices for oil and maize, had convinced them of the need for additional external support, including an augmentation of the access under the PRGF arrangement.

17. Further to terms of trade and weather-related shocks, the authorities recognized that the main risks to the macroeconomic outlook are political. An intensification of political tensions within the ruling coalition or other security-related shocks could hold back private investment. On the upside, the economy would respond much faster to a speedy resolution of constitutional reform and an acceleration of reforms, particularly in the financial and parastatal sectors.

\section{B. Fiscal Policy}

18. The authorities stressed that, over the medium term, fiscal policy would continue to balance the commitment to debt sustainability with the need to support the poverty reduction strategy. They pointed out that the slow resumption of donor budgetary assistance necessitated a near-term adjustment of the fiscal strategy (Box 5). The program for 2004/05 therefore emphasizes revenue improvements (see below), a more modest expansion in spending on poverty reduction outlays, and domestic borrowing of 2.5 percent of GDP, (Table A and Figure 3). ${ }^{1}$ Against the background of a projected increase in interest rates and, thus, lower growth in private credit, absorption of the government borrowing requirementabout 50 percent of projected financial savings - would be consistent with the targeted decline in underlying inflation.

Table A. Kenya: Fiscal Framework for 2004/05

(In percent of GDP)

\begin{tabular}{lrrrr}
\hline & $2002 / 03$ & $2003 / 04$ & \multicolumn{2}{c}{$2004 / 05$} \\
\cline { 4 - 5 } & & (Prel.) & $\begin{array}{r}\text { Initial } \\
\text { projection }\end{array}$ & $\begin{array}{r}\text { Program } \\
\text { projection }\end{array}$ \\
\hline Revenue & 20.5 & 21.7 & 22.8 & 21.4 \\
Expenditure and net lending & 20.9 & 23.5 & 29.2 & 25.5 \\
$\begin{array}{l}\text { Overall fiscal balance (before } \\
\text { grants) }\end{array}$ & -5.4 & -1.7 & -6.5 & -4.1 \\
Domestic borrowing & & & & \\
Stock of domestic debt & 4.6 & 0.8 & -0.5 & 2.5 \\
& 24.3 & 22.2 & 24.8 & 22.3 \\
\hline
\end{tabular}

Sources: Kenyan authorities and Fund staff.

\footnotetext{
${ }^{1}$ Consistent with the Constitution and the Exchequer and Audit Act, which authorizes the Minister of Finance to adjust the budget in line with available resources, departmental releases are now based on the agreed fiscal framework.
} 
19. The staff agreed that the emphasis on strengthening revenue collection was an appropriate response to shortfalls in donor budget support. ${ }^{2}$ Current efforts to improve revenue collection aim at partially offsetting the lower budgetary resources in 2004/05 stemming from shortfalls in donor assistance. With extensive FAD technical assistance, the

\section{Box 5. Kenya: Fiscal Strategy Underpinning the PRGF Arrangement}

\section{Overall fiscal stance}

- $\quad$ Gradually reducing the ratio of the net present value (NPV) of total debt to GDP.

- $\quad$ Strengthening the structural fiscal position.

- Dealing decisively with contingent-fiscal liabilities that might arise from pensions, the financial sector, and local governments.

\section{Reorienting the budget toward pro-poor expenditure}

- Increasing spending on priority social and economic outlays.

- Reducing the ratio of the wage bill in total spending by continuing to implement civil service reforms, including new wage-setting mechanism for public employees.

- $\quad$ Tackling excessive budget rigidity.

Upgrading the expenditure management system

- $\quad$ Strengthening the connection between annual budgets and the medium-term expenditure framework.

- Reforming the procurement system.

- Enhancing the modalities for monitoring, tracking, and reporting pro-poor budget operations.

- Refining further the system for controlling expenditure commitments, particularly the management of utility bills and the accountability of controlling officers.

- Improving budget classification.

- $\quad$ Establishing a modern, consolidated system of financial reporting by state enterprises.

Strengthening revenue performance

- Maintaining revenue as a proportion of GDP of at least 21.5 percent of GDP.

- Broadening the tax base by removing most exemptions.

- Taxing a larger share of informal transactions.

- Simplifying and rationalizing the tax system.

Capacity building

- Building budget and economic management capacity of the Ministry of Finance.

- Strengthening budget management and accounting capacity of key line ministries

\footnotetext{
${ }^{2}$ Revenue performance would be equivalent to the 2003/04 outcome, when account is taken of the 0.3 percent of GDP revenue loss from the introduction of the East African Community (EAC) common external tariff (CET) in January 2005. Revenue would, however, fall short of the original program projection because of the negative revenue effects of lower investment. In addition, the composition of output would differ significantly from the structure envisaged last year.
} 
authorities are implementing measures aimed at a further rationalization and computerization of key departments, as well as an upgrading of tax payments systems (MEFP para. 15).

\section{The Kenyan representatives explained that uncertain donor support} underscored the urgency of expenditure restructuring in favor of poverty outlays. First, the program reverses recent trends toward increased current spending by raising capital outlays from 2.7 percent of GDP in 2003/04 to 4.4 percent of GDP in 2004/05, with allocations for roads, health, and agriculture - priority poverty and growth-oriented outlaysaccounting for the bulk of the increase. Second, new transitional procurement rules that have institutionalized competition for government supplies are expected to lower supply costs. Third, several new initiatives discussed in the MEFP (para. 16) will, over the medium term, lower spending on noncore activities. These include the rationalization of diplomatic representation and the reform of the tertiary education funding system. Finally, the problem of budget rigidity is being addressed partly through the new wage-setting mechanism for public employees (see below) and through the targeted lowering of domestic debt.

\section{The modernization of the PEM system that has recently been initiated is} essential to put the economy on a sustainable, rapid, and poverty-reducing growth path. With the assistance of the World Bank and the Fund, the government is taking the steps in this area that are outlined in the MEFP (para. 17).

\section{The government considers the upgrading of the civil service and capacity} building of key organizations as important for poverty reduction and public sector modernization. Central government administrative staff was cut from 272,000 in 1993/94 to about 190,000 in 2003/04, while the number of teachers rose from 210,724 to 236,000 (see accompanying Selected Issues Paper).The authorities intend to continue the general hiring freeze for the civil service, but will apply it flexibly, with half of the vacancies created through natural attrition to be filled primarily by poverty reduction priority sectors. They explained that, in addition to the hiring freeze, the government intended were to reduce the wage bill as a proportion of total revenue, by explicitly linking future wage awards to revenue growth, and to lower executive compensation by aligning it to comparable pay in other African countries and the private sector. For the professional cadre, the plan was to move gradually to an incentive-based wage.

\section{Monetary and Exchange Rate Policies}

23. Discussions on monetary policy focused on an exit strategy from the negative real interest and high inflation environment. The staff pressed the authorities for a speedy tightening of monetary policy to reduce inflation. In response, the authorities explained that they had thus far tightened policy gradually because of concerns about a possible loss of control over monetary aggregates if banks responded to potential capital losses from sharp interest rate rises by rediscounting their large holdings of government bonds. However, after a November 2004 MFD technical assistance mission suggested that the risks of a significant flight from bonds was small, the central bank accelerated monetary tightening in the context of a reserve money program designed to reduce broad money growth to 7.5 percent in 
2004/05 (MEFP para.19). ${ }^{3}$ The authorities also noted that the use of reserve money performance targets would make it easier to control monetary aggregates.

\section{Competitiveness Issues}

24. Accelerating the pace of growth to reduce unemployment and poverty requires a strengthening of Kenya's competitive position. Discussions with the business community revealed that this required concerted action in a range of areas to improve the business climate. ${ }^{4}$ A number of indicators, including high labor costs (see below), point to a marked decline in Kenya's competitiveness in recent years. Moreover, a review of Kenya's real effective exchange rate (REER) indicates that the REER may be more appreciated than suggested by its economic fundamentals, such as productivity changes and commodity prices. Accordingly, the staff suggested more flexibility in the management of the floating exchange rate system. In response, the authorities noted that the shilling would continue to be allowed to respond to market forces and that interventions would be solely for the purpose of smoothing short-run fluctuations that are not related to changes in fundamentals.

25. The government has initiated actions to streamline the regulatory framework, which should promote investment. The authorities explained that the Investment Promotion Bill that is now before Parliament aims to reduce business costs in Kenya by eliminating unnecessary bureaucratic procedures and rationalizing the regulatory system (Table B provides information on comparative indicators of doing business in Kenya).

26. A change in the wage-setting system could help restore competitiveness.

Available data indicate that average nominal dollar wages for unskilled workers in Kenya are above those in other EAC member countries and competitor countries, such as India and China. In addition, annual average real wage increases in the manufacturing sector have averaged 8 percent during the past five years while labor productivity has reportedly fallen. The real minimum wage has also been raised markedly in the past five years, at a time of declining per capita real GDP. These developments have raised concerns that the wagesetting system may be a major contributor to the erosion of Kenya's competitiveness. The authorities attributed the recent inflation in wages to the use of headline consumer price inflation as the primary criterion for determining wage awards. They agreed that the intended reversion to productivity-based awards should greatly help to dampen awards.

\section{E. Governance}

\section{The government has continued to implement a broad-based strategy to enhance} governance amid an intensive debate on some important gaps in the anticorruption

\footnotetext{
${ }^{3}$ The three-month treasury bill rate has been increased from 2.75 percent in September 2004 to 5.2 percent in November.

${ }^{4}$ The government is addressing many of these issues in the context of parallel World Bank programs.
} 
agenda. In the recent public debate, some of Kenya's main donors have identified important gaps in the governance agenda, including (a) inadequate funding of governance institutions; (b) shortcomings in the prosecution branches; and (c) weaknesses in the management of security procurements. Nevertheless, the appointment of the top management of the KACC and recent strengthening of the police and Office of the Comptroller and Auditor General have bolstered the investigative branches of Kenya's governance system. Translation of these efforts into a corresponding increase in successful prosecutions of corruption cases hinges on the effectiveness of the Attorney General's office. Modification of the Public Office Ethics Act to provide for the regular review of the asset declarations of prominent officials would close a major loophole in the governance strategy (MEFE para. 19). The governance agenda includes also a comprehensive set of actions designed to improve the transparency of the budget and financial management systems.

28. The authorities acknowledged that more steps were needed to address corruption and considered ongoing reforms to produce, disseminate, and audit public accounts to be critical in this regard. They noted that first, the capacity-building program in the Ministry of Finance would facilitate the production, on a timely basis, of more comprehensive and better articulated annual and medium-term budgets and, hence. a more transparent budget-management process. Second, the ongoing strengthening of public sector accounting capacity and systems and the requirement that the government produce final accounts within four months and accounts audited by the Auditor General within nine months of the end of the fiscal year would greatly facilitate parliamentary scrutiny of public operations. Moreover, the Auditor General's report will be a key reference point for the investigative work of the KACC. Third, the recent restructuring of the Auditor General's office had enabled it to begin to conduct more timely audits of the accounts of state bodies, including parastatals and local governments, and to play a more proactive role in investigations of potential misuse of public funds, as borne out in the Anglo-Leasing case ${ }^{5}$ and the recent review of the improper contracting by government officials of commercial debt. Staff agreed that these steps, with other measures outlined in para. 20 of the MEFP, would represent important progress toward closing the remaining governance gaps.

29. The authorities confirmed that their anticorruption strategy also emphasized the building of sound governance institutions. They noted that, at the political level, the draft constitution includes many provisions designed to enhance inclusiveness and transparency in the conduct of public business, including by devolving more authority to local governments. The draft constitution also enshrines the independence of the judiciary and the public prosecution office. The authorities confirmed that they were taking steps to address weaknesses in the prosecution branch by hiring more and better-qualified staff. With regard to the Anglo-Leasing scandal, they explained that the actions they had taken to address the issues raised by the scandal were consistent with the law and, in their view, considerably

\footnotetext{
${ }^{5}$ This scandal involved the purchase of equipment at prices well above market, and without following proper procurement procedures.
} 


\begin{tabular}{|c|c|c|c|c|c|}
\hline & Tanzania & Uganda & Kenya & China & India \\
\hline Monthly earnings of unskilled production workers (U.S. dollars) & 51.70 & 57.48 & 99.20 & 85.00 & $\$ 50.00$ \\
\hline $\begin{array}{l}\text { Unit labor cost } \\
\text { (ratio of wages to value added) }\end{array}$ & 0.39 & 0.39 & 0.36 & 0.32 & 0.27 \\
\hline Cost of electricity (US\$ per KwH) & $\ldots$ & $\ldots$ & 0.10 & 0.10 & 0.09 \\
\hline Share of paved roads in total roads & 4 & 7 & 12 & 91 & $46 \%$ \\
\hline $\begin{array}{l}\text { Telecommunication costs } \\
\text { (U.S. dollars for a three-minute international call) }\end{array}$ & 9.60 & 3.63 & 5.80 & $\ldots$. & $\$ 3.20$ \\
\hline $\begin{array}{l}\text { Transparency International Corruption Perceptions Index } \\
\text { (higher number indicates greater corruption) }\end{array}$ & 92 & 113 & 122 & 66 & 83 \\
\hline $\begin{array}{l}\text { Share of investors who perceive the investment climate } \\
\text { in the country to be deteriorating versus share who perceive } \\
\text { it to be improving }\end{array}$ & 7 vs. 44 & 5 vs. 47 & 33 vs. 30 & $\ldots$ & n.a. \\
\hline
\end{tabular}

Sources: World Bank Investment Climate Assessment for Kenya (forthcoming); World Development Indicators database; and

United Nations Industrial Development Organization (UNIDO) Africa Foreign Investor Survey 2003. 
stronger than the recent responses of many other African countries to similar events. In this regard, all the senior officers involved in the scandal have been suspended; the KACC was asked to initiate detailed investigations, which are still underway and a parliamentary inquiry was also conducted. While the authorities argued that their efforts were securing important further progress on governance, they were nevertheless concerned that, as with other priority programs, the difficult budgetary situation constrained their ability to fund key anticorruption agencies adequately. They were optimistic that donor assistance would help alleviate the problem.

\section{F. Public Enterprise Restructuring and Privatization}

30. Delays in forging a consensus to restructure and privatize parastatals have slowed reforms in this area. The government believes that, in the past, political and rentseeking considerations played a central role in privatization decisions. It has reaffirmed its strong commitment to privatization and enterprise reform, but wants to build broad parliamentary support for the reforms. The authorities therefore intend to rely on the Parliament's planned consideration, in the near future, the policy paper "Privatization of State Corporations" to present the rationale for the planned reforms before they resubmit the Privatization Bill to Parliament. Pending the enactment of the Privatization Bill, the focus will be on preparing enterprises for privatization, including by conducting detailed reviews of the financial position of key parastatals.

\section{G. Financial Sector}

\section{The authorities agreed that major efforts were needed to strengthen the}

financial sector. They indicated their intention to tighten loan classification and provisioning in January 2005, in line with the recommendations of the recently concluded FSAP (Box 6), and to strengthen the CBK's Banking Supervision Department (BSD). While welcoming these actions, the staff stressed the need for prompt follow-up actions on future recommendations of the BSD in order to address banking sector problems more decisively and promptly. The staff also welcomes the proposed amendments to the CBK and Banking Acts currently before Parliament, which seek to transfer bank regulatory functions from the Ministry of Finance to the $\mathrm{CBK}$ and to remove the provisions that give the Minister of Finance authority to control interest rates (MEFP para. 22).

\section{The authorities pointed out that large interest rate spreads inhibited bank}

lending and economic growth. They explained that while the lowering in mid-2003 of required banks' reserves had helped reduce interest rates and interest rate spreads, financial intermediation, particularly to small and medium-sized firms, continued to be hindered by relatively large interest rate spreads. Banks have attributed the high spreads to low business volumes, legal and administrative impediments to the enforcement of contracts, and inefficiencies in the labor market that had contributed to significant increases in labor costs. While stressing the importance of addressing these structural problems, the staff pointed out that interest rate spreads in Kenya were not significantly different from those prevailing in countries at a similar stage of development. 
33. While welcoming the recent decline in the NPLs, the staff stressed that decisive steps to address the problem of public sector banks were needed to resolve the NPL problem. The authorities noted that most of the NPLs of public banks were on account of old government-intermediated credits, as well as from banks' failure to enforce contracts under the old regime because of legal, political, and judicial hurdles. They therefore felt that under the new political order, policies needed to focus on the restructuring of public banks. With regard to the privatization of public banks, they indicated that caution was needed, as

\section{Box 6. Main Conclusions of the Financial Sector Assessment Program (FSAP)}

The FSAP found that although the major elements of a well-developed financial system are in place, financial intermediation is weak. The main findings are presented below:

- $\quad$ The legal and judicial framework needs to be strengthened to promote financial sector development.

- $\quad$ The banking system has high levels of nonperforming loans and low capitalization and is vulnerable to shocks that could endanger its stability.

- $\quad$ To reduce the vulnerabilities, supervisors need to intervene rapidly to deal with problem financial institutions.

- $\quad$ Highest priority should be given to restructuring banks and divesting government stakes in the banking sector; also, the government should reevaluate its role in the development finance sector.

- The government is appropriately seeking to bolster the microfinance sector and, thereby, access to financial services for underserved segments of the population.

- $\quad$ The authorities are urged to move ahead with reforms of the payments and settlements systems.

A program of technical assistance is under way to assist the authorities in their financial sector reform efforts.

experiences in Kenya and other African countries indicated that sales of banks to multinationals reduced bank penetration to rural areas, and lowered further access to credit of small and medium-sized firms. The authorities therefore favored a bank privatization program that would be explicitly linked to the realization of predetermined thresholds of banking service penetration to rural communities and disadvantaged groups. In line with the findings of the recent FSAP, the staff noted that, with over 40 commercial banks, Kenya was well served with banking services and that current efforts to foster microfinance institutions nationally would help support the rural sector. In addition, the staff supported the World Bank's call for a comprehensive financial sector strategy paper to better elaborate the issues in the sector, including the role of development banks. 


\section{H. External Trade and Regional Integration}

\section{The EAC common external tariff, taking effect in January 2005, is at the center} of government initiatives to promote external trade. The EAC customs union will lower the number of Kenya's tariff bands from five to three, the maximum tariff rate from 35 to 25 percent, and the average tariff from 17.2 percent to 10.9 percent. Data on average tariff and other tax rates among the EAC members and prospective members are provided in Table C. ${ }^{6}$ The fiscal and trade impact of the CET for Kenya will, in part, depend on the effects of the temporary protection that has been accorded to Tanzanian and Ugandan producers for a specified list of commodities for five years, ${ }^{7}$ as well as on how potential conflicts with other regional trade agreements (COMESA in the case of Kenya) are resolved. The members of the EAC have agreed to maintain, beyond January 2005, existing legal obligations until these potential conflicts are settled. The authorities indicated that they expected Kenya to suffer some near-term losses from the implementation of the CET as domestic production is replaced by cheaper imports. Over the medium term, as economic reforms are deepened, they expected the economy to benefit from the lowering of tariffs, which would help foster a more competitive environment and an expansion in exports.

\footnotetext{
${ }^{6}$ Kenya's nontariff barriers to trade (NTBs) include suspended duties on petroleum products that can be levied and removed on an ad hoc basis identified in business surveys as trade barriers. Current efforts at improving customs administration and simplifying import procedures; will facilitate trade.

${ }^{7}$ Tanzania and Uganda will eliminate tariffs on all imports except those on an agreed number of commoditiesTanzania 906, and Uganda 426 tariff lines - for which the tariff will be gradually reduced to zero within up to five years.
} 
Table C. Comparative Tax Rates in the East African Region

\begin{tabular}{|c|c|c|c|c|c|}
\hline & Kenya & Tanzania & Uganda & Rwanda & Burundi \\
\hline $\begin{array}{l}\text { Personal income tax } \\
\text { (in percent) }\end{array}$ & $\begin{array}{r}10 \%-30 \% \\
(5 \text { brackets) }\end{array}$ & $\begin{array}{r}0 \%-30 \% \\
\text { (5 brackets) }\end{array}$ & $\begin{array}{r}0 \%-30 \% \\
\text { (4 brackets) }\end{array}$ & $\begin{array}{r}0 \%-35 \% \\
\text { (5 brackets) }\end{array}$ & $\begin{array}{r}0 \%-60 \% \\
\text { (12 brackets) }\end{array}$ \\
\hline $\begin{array}{l}\text { Corporate income tax } \\
\text { (in percent) }\end{array}$ & $\begin{array}{c}30 \\
(37.5 \text { for } \\
\text { branches of foreign } \\
\text { companies })\end{array}$ & 30 & 30 & 35 & 35 \\
\hline Valued added tax & 16 & 20 & 17 & 18 & - \\
\hline $\begin{array}{l}\text { Excise tax on Gasoline } \\
\text { (in U.S. dollars; for Rwanda, }\end{array}$ & US\$ 0.25 & $\begin{array}{c}0.14 \\
(0.11 \mathrm{VAT})\end{array}$ & 0.34 & 37 & n.a. \\
\hline $\begin{array}{l}\text { Ex percent } \\
\text { Excise tax on Cigarettes } \\
\text { (in percent; for Tanzania } \\
\text { in U.S. dollars) }\end{array}$ & $\begin{array}{l}130 \text { or specific } \\
\text { (whichever is } \\
\text { higher applies) }\end{array}$ & 7.62 & 150 & 60 & 58 \\
\hline $\begin{array}{l}\text { Excise tax on Beer (in U.S. } \\
\text { dollars; for Rwanda and Burundi, } \\
\text { in percent }\end{array}$ & 0.47 & 0.22 & $\begin{array}{l}0.03 \\
(0.01 \text { for } \\
\text { local beer })\end{array}$ & 57 & 51 or 31 \\
\hline $\begin{array}{l}\text { Unweighted average tariff } \\
\text { (in percent) }\end{array}$ & 16.6 & 12.5 & 7.3 & 23.5 & 18 \\
\hline
\end{tabular}

\section{External Financing Requirements}

35. Kenya's projected external financing requirements are high but appear achievable, based on the experiences of other low-income countries that have implemented strong policy reforms (Table 8). The World Bank has committed US\$870 million in its Country Assistance Strategy base case for Kenya for FY 2004-07. The African Development Bank and the European Union are expected to provide significant budget support under the base case scenario, with other donors also contributing some budget support. The next Consultative Group meeting for Kenya is scheduled for early 2005. Reflecting the scaling down of concessional financing since the last staff report, Kenya's external debt (in percent of GDP) remains on a declining trend (Table 9) and well below the thresholds under the enhanced HIPC Initiative. Updated debt sustainability analysis (Appendix IV) confirms that, under the revised program framework, external debt sustainability improves compared to the assessment undertaken in November 2003. The projected increase in domestic borrowing over the near term would only marginally increase the NPV of central government debt relative to GDP.

36. As a result of an audit of its external commercial contracts undertaken for governance reasons, Kenya has recently begun to accumulate some arrears on these obligations. The government is establishing an escrow account into which the debt service due on commercial credits will be placed, to be used to settle fully all legitimate debts. In accordance with the Fund's policy on lending into arrears, financing assurances reviews will be conducted under the PRGF arrangement for each disbursement as long as arrears to private creditors exist. Reflecting that total external obligations to private creditors are low (an estimated US\$296 million at end-2004, or 5.6 percent of Kenya's total external debt stock), the amount of the arrears expected to be accumulated while the audit is being 
conducted would be relatively small. The government has expressed its intention to complete the audit in a timely fashion, and, as noted above, has established an escrow account for the unpaid amount under those contracts. In light of these considerations, the staff is of the view that the Kenyan authorities have shown good-faith intentions to resolve the problem.

\section{Details of Kenya's Paris Club rescheduling for January 2004 are provided in}

Box 7. To date, Kenya has signed bilateral agreements with five Paris Club creditors. The authorities report only one nonParis Club creditor with pre-cut-offdate debt; little progress has been made in normalizing debt with this creditor. As discussed below, a modest amount of nonrescheduled arrears to Paris Club creditors were settled by November 2004 rather than by the due date of July 1, 2004. Table 9 indicates the impact of the Paris Club rescheduling on Kenya's debt service ratios over the consolidation period of 2004-07.

\section{First REVIEW OF THE ARRANGEMENT}

38. As indicated in the attached MEFP, with regard to the end-December 2003 and end-March 2004 performance criteria (PC) under the program, Kenya met all of the structural PC, albeit some with delays, and all but two of the quantitative PC (MEFP Tables 1 and 2). ${ }^{8}$ The structural PC on the completion by end-December 2003 of an audit of the financial position of the National Social Security Fund (NSSF) was observed in February 2004, and that on the submission to Parliament by end-March 2004 of the Banking Act Amendment Bill was met in June 2004. With regard to the PC on the completion of an audit of the stock of pending bills and the adoption of measures to provide for their clearing, a financial audit was completed by December 2004, but the authorities are awaiting the results of a legal audit of the pending bills before finalizing the clearance plan. The quantitative continuous PC limiting the contracting of nonconcessional external long-term debt was breached when financing contracts for two security-related procurements were signed in December 2003. Subsequently, the authorities cancelled the contracts, thus correcting the breach. The continuous quantitative PC on the nonaccumulation of external arrears was breached for June 30, 2004, on two categories of arrears. There was a late payment of the nonrescheduled component of Paris Club arrears existing at the time of the January 2004 rescheduling, which was required to be settled by July 1, 2004, but was not fully cleared until November 2004. Some late payments and arrears have also resulted from the suspension of payments while an audit of all central government commercial credits is conducted.

39. The MEFP spells out the structural and quantitative performance criteria and benchmarks (MEFP Tables 3 and 4) to be used in monitoring performance under the 2004/05 program, with target dates of end-March and end-September 2005. ${ }^{9}$ Targets for the latter are indicative and will be firmed up in discussions for the second review of the

\footnotetext{
${ }^{8}$ It also met all but two of the indicative target/performance criteria for end-June 2004.

${ }^{9}$ Previously, the program had end-December and end-June targets.
} 
PRGF arrangement, when the fiscal framework for 2005/06 will be agreed. The government is taking a number of actions prior to the issuance of the staff report on the first review of the PRGF arrangement.

\section{OTHER ISSUES}

The quality and timeliness of Kenya's reporting of economic and financial data are adequate for surveillance and program-monitoring purposes, notwithstanding the need for improvement in several areas. The staff expressed concerns about the problems involved in monitoring fiscal performance, in particular domestic arrears. The authorities indicated that they were addressing these shortcomings with assistance from AFRITAC. Kenya is a participant in the GDDS.

40. Anti-money-laundering legislation is being drafted; it is expected to be presented to parliament by mid-2005.

41. The CBK has made progress in implementing the recommendations of the $\mathbf{2 0 0 3}$ Safeguards Assessment. The authorities are committed to implementing all the recommendations of the staff (MEFP, para. 27). 


\section{Box 7. Kenya's Paris Club Rescheduling}

The Paris Club met on January 14-15, 2004, to consider Kenya's request for debt relief in the context of the country's PRGF arrangement. Kenya requested and received Houston rescheduling terms, despite its IDA-only country status. The agreement rescheduled US\$350 million arrears and maturities falling due in the consolidation period of January 1, 2004 , to December 31, 2006, on a total debt service of US\$484 million due to Paris Club creditors during this period. Assuming an additionalUS\$52 million in comparable treatment from other creditors, the relief would total US\$402 million. The cutoff date remained December 31, 1991, as it did for Kenya's last Paris Club rescheduling in November 2000.

Under the agreement, official development assistance (ODA) credits are to be repaid over 20 years with 10 years grace, at interest rates at least as favorable as the concessional rates applying to those loans, and commercial credits are to be repaid over 15 years, including 5 years of grace, with progressive repayments at an appropriate market interest rate. One hundred percent of service on not-previously rescheduled debt was rescheduled, while debt service on previously rescheduled debt was treated less generously. ${ }^{1}$ Sixty percent of arrears to the Paris Club as of end-2003 that had not previously been rescheduled were rescheduled.

The Paris Club creditors and the Kenyan authorities considered that this rescheduling, the first under the Evian Approach, should allow Kenya to graduate from future Paris Club treatments. The deadline for signing bilateral agreements has been extended until December 31, 2004.

${ }^{1}$ For previously rescheduled debt, 100 percent of total debt service (principal and interest) due in 2004 and 100 percent of principal and 50 percent of interest due in 2005 was rescheduled, while no debt service on previously rescheduled debt due in 2006 was treated.

\section{Staff Appraisal}

42. Kenya has made significant gains during the past year. Broad agreement has been reached on most of the constituent elements of the new constitution, and a consensus appears to be emerging on the remaining contentious issues; a rebound in economic activity is under way; the recent sharp growth in Kenya's domestic debt has been arrested; the governance architecture and key institutions have been upgraded and enforcement of elements of the apparatus strengthened; and revenue administration has been enhanced.

43. However, major challenges remain. The political outlook is uncertain; inflationary pressures have increased with monetary policy responding too slowly; the restructuring of the fiscal position necessary for the realization of the MDGs has barely begun; some important gaps in the governance architecture have yet to be filled; implementation of key structural reforms has been slow, constrained, in part, by weak international support; and concerted actions are needed to address the recent erosion in Kenya's international competitiveness. 
Medium-term growth hinges on deepening reforms and addressing capacity constraints, and the resumption of strong donor support if the authorities are to fulfill their appropriately ambitious vision for Kenya.

44. Decisive actions are needed to reduce inflation, which requires an early tightening of monetary policy. The continued tightening of monetary policy envisaged under the program should bring real interest rates back to positive levels. Otherwise, the persistence of inflationary pressures could affect expectations and complicate the authorities' ability to resist wage pressures and the restoration of a competitive economic environment. The staff supports the authorities' decision to maintain the current managed floating exchange rate system that has served Kenya well in responding to external shocks.

\section{Fiscal policy faces the challenge of balancing the commitment to fiscal} consolidation with the support for poverty reduction programs. In particular, managing the near-term temporary increase in domestic borrowing and domestic debt will be a major challenge. The increase in domestic borrowing planned for 2004/05 appears reasonable in view of the large shortfalls in donor inflows and likely contractionary effects of the expected rise in interest rates. For the following year and over the medium term, the budget should begin the consolidation. In this regard, the wide range of measures taken by the authorities to strengthen revenue collection is a welcome development. This will need to be supported by decisive efforts to control and prioritize expenditure programs, determined steps to cut nonessential outlays, and the resumption of strong donor support.

\section{Improved expenditure management is essential to achieving rapid poverty} reduction and economic growth. The authorities' program outlines promising steps in this direction, including the strengthening of the expenditure commitment control system, an early start and lengthening of the budget preparation period, enhanced the links between the annual budget and the MTEF, a strengthening of the MTEF as a budget management tool, and consolidation of the budget management organizational structures. These measures, if effectively implemented, should hasten the trend toward pro-poor spending and enhance budget transparency and accountability.

\section{The authorities' increased attention to actions to improve Kenya's}

competitiveness is welcome. To help address this issue, they have adopted a multifaceted strategy that includes streamlining the regulatory framework, improving essential infrastructure, reforming the labor market, and liberalizing trade. Each of these initiatives represents an important step in the right direction. However, there remains scope for further progress. The intended change in the private sector wage-setting mechanism to arrest the recent inflation in wage demands is welcome and should not be delayed.

48. The government is making progress in liberalizing external trade. The lowering of the average external tariff, in the context of the EAC, is a step in the right direction in that it will foster a more competitive domestic environment and expand exports over the medium term. 
49. The authorities are pursuing a broad-based strategy to promote good governance, but closing gaps in the anticorruption agenda remains a priority. The restructuring, during the past year, of the police, the judiciary, the Office of the Ombudsman, and the Auditor General are noteworthy steps. In addition, the recent establishment of the KACC is an important milestone. The authorities' program also includes other steps that would go a long way toward filling important gaps in the present governance framework, including the regular verification of asset declarations of senior officials and the production of budget outlook papers, which should make parliamentary oversight of fiscal operations more effective. Long overdue steps have now been taken to strengthen prosecution capacity, which should help restitution and deterrence efforts, and to increase oversight over military procurement. Steadfast implementation, including enforcement, will be key to the success of the fight against corruption, and donors have an important role to play in supporting key governance institutions.

50. Public enterprise restructuring and privatization are essential to promoting strong economic growth. The delay in the enactment of the Privatization Bill has helped foster inefficient enterprises and slowed economic growth. The authorities need to redouble their efforts to secure parliamentary support for the bill. Establishing the Privatization Commission is important to managing privatization transparently; transparency is a key ingredient in building and maintaining public support for the reforms. The decision to begin a detailed systematic assessment of the financial position of key parastatals is welcome. In addition to establishing the magnitude of government contingent liabilities, the assessments should help expedite the retrenchment process.

51. The ongoing reform of the financial system should help enhance financial intermediation. The program of transferring the regulatory framework from the government to the $\mathrm{CBK}$, revising banking legislation to remove the restrictive provisions for banks' pricing decisions, tightening prudential regulations, and restructuring state-owned banks is essential to strengthening financial sector performance and increasing financial intermediation. The recent improvement in indicators of banking sector performance, including the fall in NPLs, is a welcome development. However, resolution of the NPL problem will require the restructuring and privatization of publicly owned banks. The staff supports the authorities' focus on promoting financial intermediation in rural areas, as well as on increasing the access of small and medium-sized firms to formal sector financial resources. This will require both a broadening of the scope and outreach of microfinance institutions and a more coherent and targeted strategy for promoting development finance institutions.

\section{In light of Kenya's performance under the program, the staff recommends} completion of the first review under the PRGF arrangement. The staff supports the authorities' request for three waivers for nonobservance of structural PC and two waivers for nonobservance of quantitative $\mathrm{PC},{ }^{10}$ as well as the authorities' request for a rephasing of

\footnotetext{
${ }^{10}$ The circumstances of these waivers are described in the section on program monitoring.
} 
disbursements. It also supports the authorities' request for an augmentation of access under the PRGF arrangement of SDR 50 million.

53. It is proposed that the next Article IV consultation with Kenya be held in accordance with the provisions of the decision on consultation cycles approved on July 15, 2002. 
Figure 1. Kenya: Real and External Developments

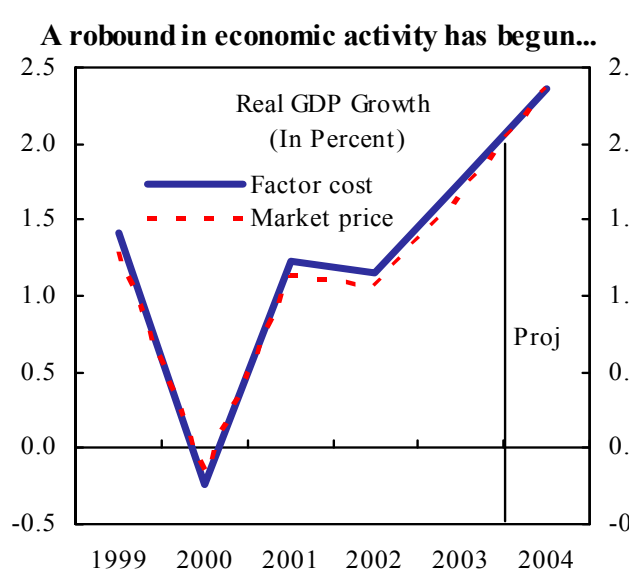

Inflation has surged recently...

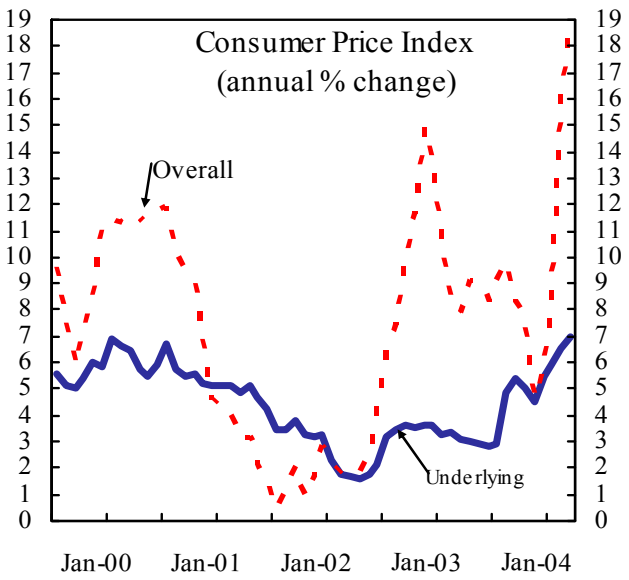

CA has worsend, as imports have picked up...

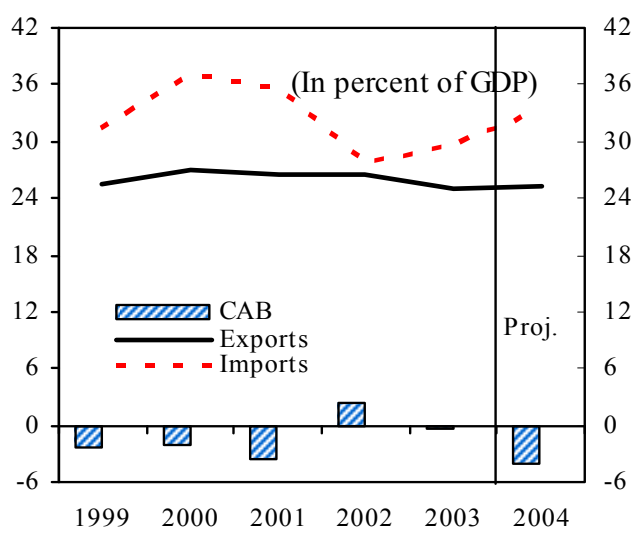

...driven mainly by a pickup in the tertiary (service) sector.

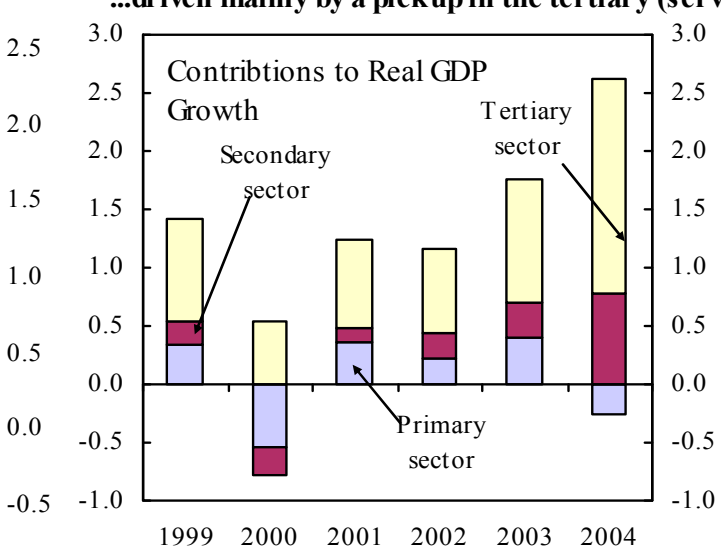

...partly due to a weakening of KSH.

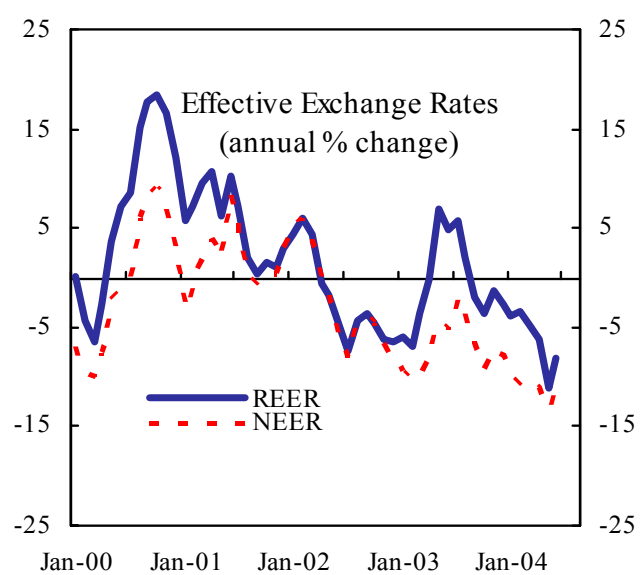

...resulting in a declining reserve coverage.

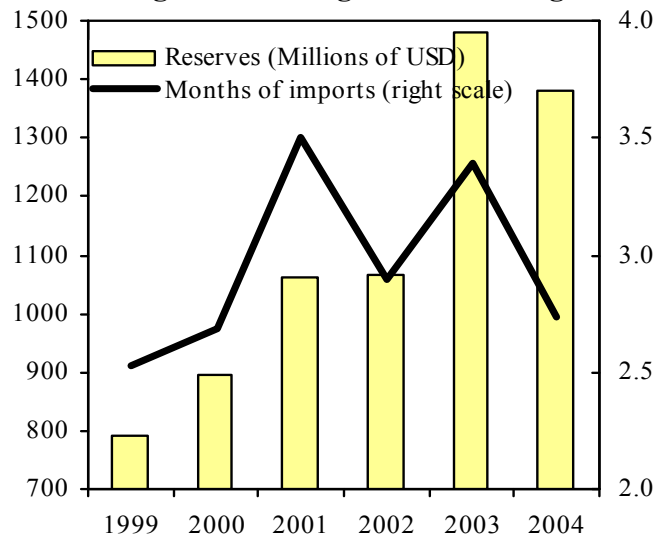

Source: Staff estimates. 
Figure 2. Kenya: Monetary and Financial Developments

Reserve money growth has exceeded program expectation...

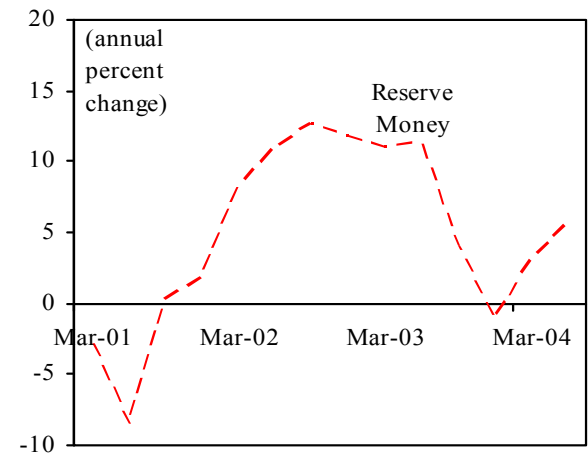

Owing to a reduction in reserve requirements, the mulitplier rose and broad money surged.

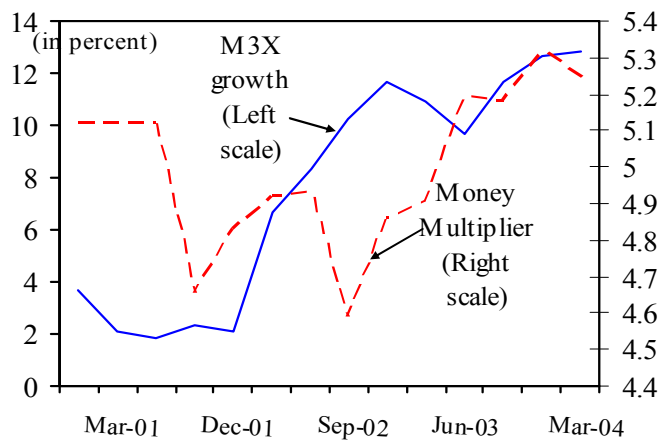

But interest rate spreads have remained high, reflecting structural weaknesses in the financial system.

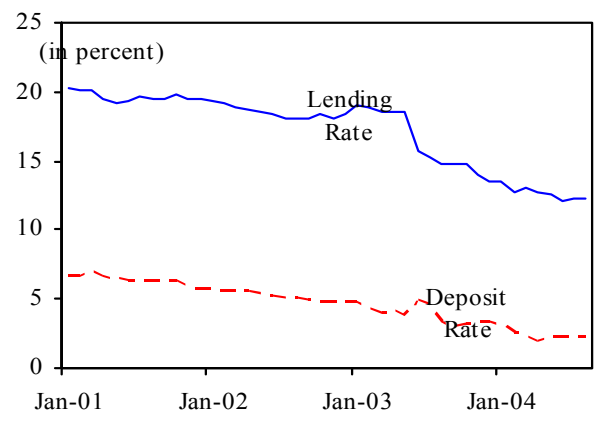

Source: Kenyan authorities. ....as the overperformance in NFA was not adequately sterilized by the slowdown in NDA.

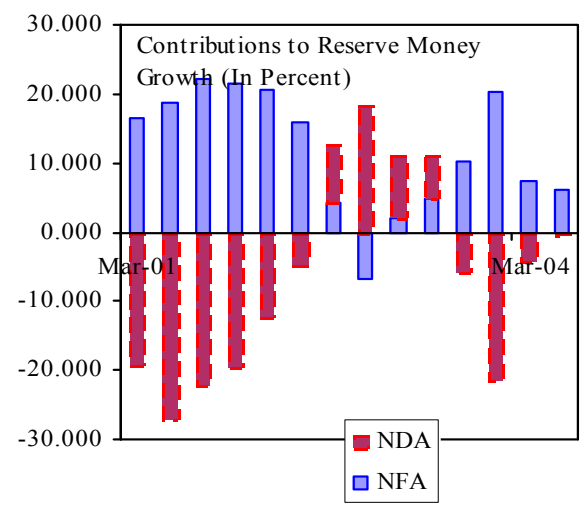

Excessive liquidity has resulted in negative real short-term interest rates.

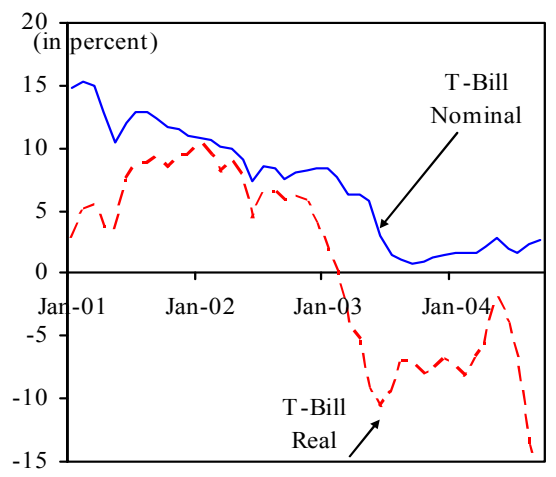

Ample liqudity and the decline in lending rates have helped private credit to grow.

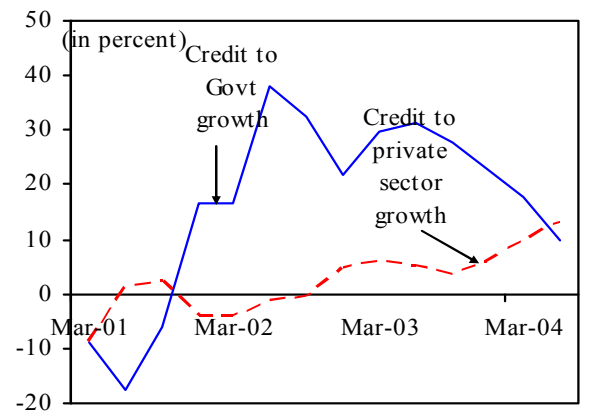


Figure 3. Kenya: Fiscal Developments

Revenue is projected to decline in $2004 / 05, \ldots$

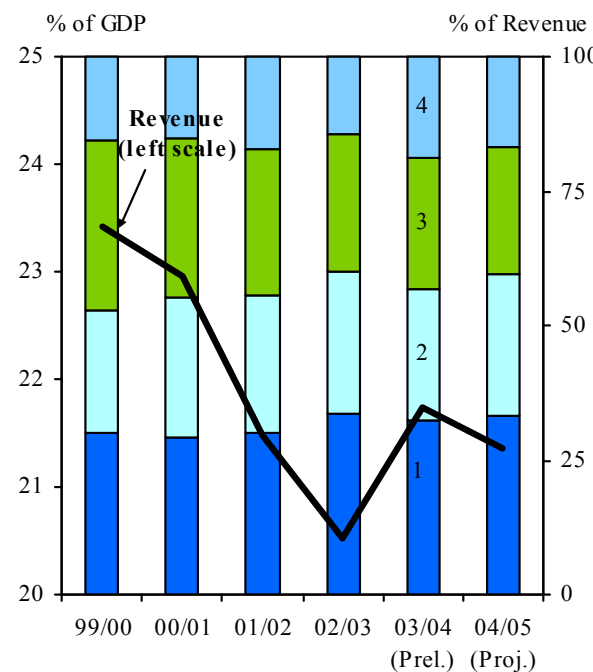

1=Income tax; 2=VAT; 3=Import and Excise duty; $4=$ other

The widening deficit in 2004/05 will be financed by an increase in domestic borrowing,...

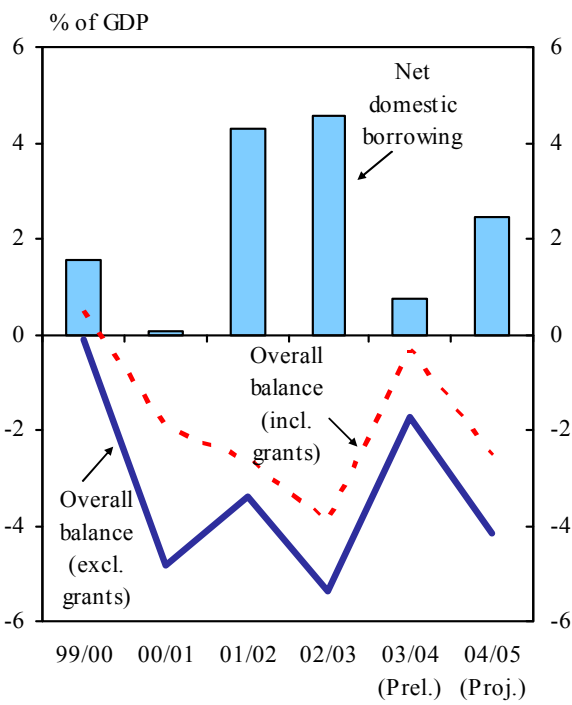

...while expenditure will rise.

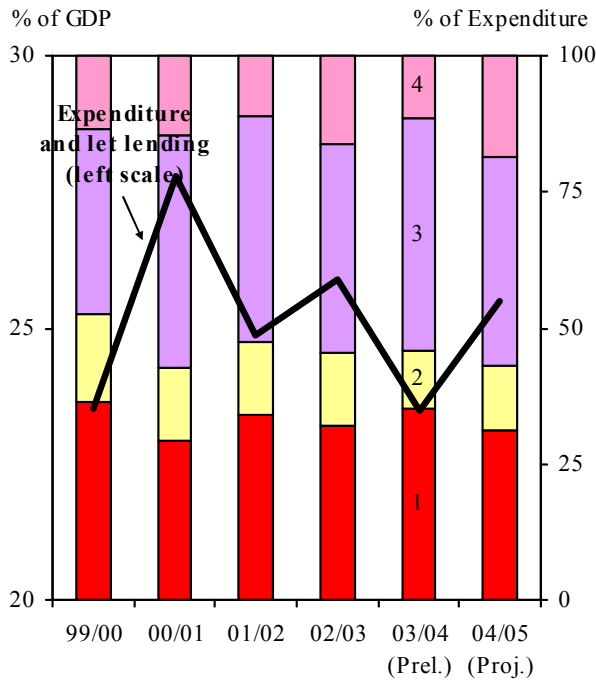

$1=$ Wages; $2=$ Interest; $3=$ Other recurrent expenditure; 4=Development and net lending

...res ulting in a small rise in domestic debt, while external debt continues to fall.

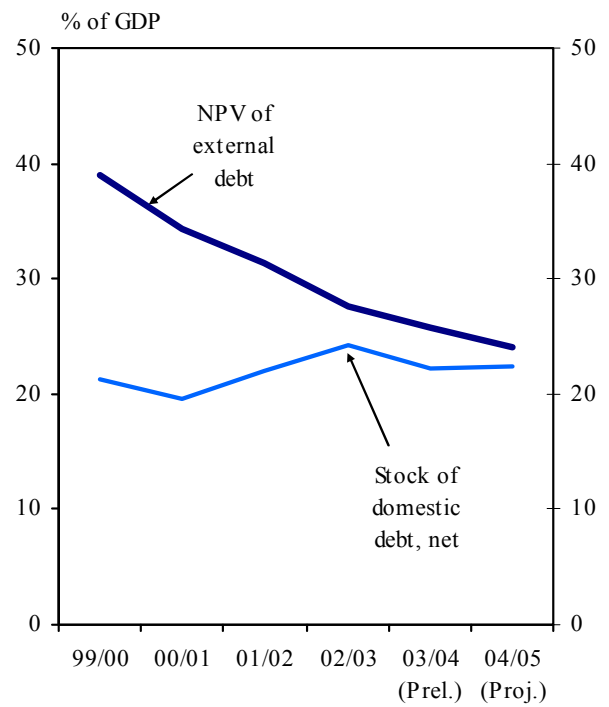

Source: Kenyan Authorities. 
Table 1. Kenya: Medium-Term Macroeconomic Framework, Base Case Scenario, 2002/03-07/08

\begin{tabular}{|c|c|c|c|c|c|c|c|}
\hline & $\underline{2002 / 03}$ & \multicolumn{2}{|c|}{$2003 / 04$} & $2004 / 05$ & \multirow{2}{*}{$\begin{array}{l}\text { 2005/06 } \\
\text { Proj. }\end{array}$} & \multirow{2}{*}{$\begin{array}{l}\text { 2006/07 } \\
\text { Proj. }\end{array}$} & \multirow{2}{*}{$\begin{array}{l}2007 / 08 \\
\text { Proj. }\end{array}$} \\
\hline & Actual & Program & Prelim. & Proj. & & & \\
\hline \multicolumn{8}{|c|}{ (Annual percentage change, unless otherwise indicated) } \\
\hline \multicolumn{8}{|l|}{ National accounts and prices } \\
\hline Nominal GDP (Market prices, in billions of Kenya shillings) & 1,027 & 1,076 & 1,162 & 1,301 & 1,423 & 1,533 & 1,651 \\
\hline GDP volume (factor cost) & 1.5 & 1.9 & 2.1 & 2.7 & 3.2 & 3.6 & 4.0 \\
\hline GDP deflator & 10.1 & 4.5 & 10.9 & 9.0 & 6.0 & 3.9 & 3.6 \\
\hline Consumer price index (annual average) & 6.6 & 4.1 & 8.2 & 12.1 & 4.1 & 3.5 & 3.5 \\
\hline Consumer price index (end of period) & 13.7 & -4.0 & 5.9 & 5.0 & 3.5 & 3.5 & 3.5 \\
\hline Consumer price index, excl. food, energy (annual average) & 2.7 & 3.0 & 3.9 & 5.6 & 3.5 & 3.5 & 3.5 \\
\hline Consumer price index, excl. food, energy (end of period) & 3.7 & 2.7 & 5.5 & 3.5 & 3.5 & 3.5 & 3.5 \\
\hline Ksh per US \$ exchange rate (end of period) & 76.4 & 76.0 & 78.9 & $\ldots$ & $\ldots$ & $\ldots$ & $\ldots$ \\
\hline Nominal effective exchange rate (- depreciation; end of period) & -5.2 & $\ldots$ & -11.1 & $\ldots$ & $\ldots$ & $\ldots$ & $\ldots$ \\
\hline Real effective exchange rate (- depreciation; end of period) & 4.7 & $\cdots$ & -8.2 & $\cdots$ & $\ldots$ & $\cdots$ & ... \\
\hline \multicolumn{8}{|l|}{ Money and credit } \\
\hline Net domestic assets (end of period) & 12.0 & 5.0 & 12.2 & 7.3 & 10.1 & 9.2 & 7.8 \\
\hline Net credit to the government (end of period) & 31.1 & 3.9 & 9.9 & 8.9 & 6.1 & 2.1 & 0.4 \\
\hline Credit to the rest of the economy (end of period) & 4.9 & 4.8 & 13.1 & 8.1 & 13.3 & 10.9 & 9.7 \\
\hline M3 (broad money, end of period) & 9.3 & 8.9 & 12.3 & 7.9 & 8.1 & 7.9 & 8.0 \\
\hline M3X (M3 plus foreign currency deposits, end of period) & 10.9 & 7.1 & 12.9 & 7.5 & 6.9 & 6.9 & 7.0 \\
\hline M4X (M3X plus nonbank holdings of government paper) & 12.1 & 7.1 & 9.3 & 9.3 & 8.7 & 6.4 & 5.7 \\
\hline Reserve money (end of period) & 11.2 & -5.4 & 5.5 & 3.8 & 6.8 & 7.0 & 7.0 \\
\hline \multicolumn{8}{|c|}{ (In percent of GDP, unldess otherwise indicated) } \\
\hline \multicolumn{8}{|l|}{ Investment and saving } \\
\hline Investment & 13.8 & 17.9 & 13.2 & 14.1 & 16.3 & 18.5 & 19.5 \\
\hline Central government & 2.0 & 4.8 & 2.1 & 3.0 & 4.3 & 4.8 & 3.9 \\
\hline Other & 11.7 & 13.1 & 11.1 & 11.1 & 12.0 & 13.7 & 15.6 \\
\hline Gross national saving & 14.7 & 13.1 & 11.7 & 9.6 & 10.6 & 13.3 & 15.2 \\
\hline Central government & -0.3 & -0.1 & 0.5 & 0.6 & 0.8 & 0.5 & -0.5 \\
\hline Other & 15.0 & 13.2 & 11.2 & 9.0 & 9.8 & 12.8 & 15.7 \\
\hline \multicolumn{8}{|l|}{ Central government budget } \\
\hline Total revenue & 20.5 & 22.2 & 21.7 & 21.4 & 21.1 & 21.9 & 22.9 \\
\hline Total expenditure and net lending & 25.9 & 27.8 & 23.5 & 25.5 & 28.6 & 28.8 & 28.8 \\
\hline Overall balance (commitment basis) excluding grants & -5.4 & -5.6 & -1.7 & -4.1 & -7.5 & -6.9 & -5.9 \\
\hline Overall balance (commitment basis) including grants & -3.9 & -3.3 & -0.3 & -2.5 & -3.5 & -3.5 & -2.9 \\
\hline Net domestic borrowing & 4.6 & 3.3 & 0.8 & 2.5 & 2.1 & 0.7 & 0.1 \\
\hline Financing gap & 0.1 & 2.7 & 0.4 & 0.0 & 0.4 & 1.4 & 2.2 \\
\hline Total donor support (grants \& loans) & 2.2 & 7.0 & 2.4 & 3.3 & 6.0 & 5.7 & 4.7 \\
\hline \multicolumn{8}{|l|}{ Balance of payments } \\
\hline Exports value, goods and services & 26.2 & 27.0 & 24.4 & 25.8 & 26.1 & 26.6 & 27.1 \\
\hline Imports value, goods and services & 29.2 & 34.8 & 30.3 & 34.7 & 35.6 & 35.3 & 34.4 \\
\hline Current external balance, including official transfers & 1.0 & -4.8 & -1.5 & -4.5 & -5.7 & -5.2 & -4.3 \\
\hline Current external balance, excluding official transfers & 1.2 & -5.0 & -2.0 & -4.9 & -6.1 & -5.6 & -4.2 \\
\hline Gross international reserve coverage & & & & & & & \\
\hline in months of next year imports (end of period) & 3.3 & 2.8 & 3.0 & 3.1 & 3.1 & 3.2 & 3.1 \\
\hline \multicolumn{8}{|l|}{ Public Debt } \\
\hline NPV of central government debt (end of period) & 51.9 & 55.6 & 49.6 & 49.6 & 49.6 & 48.5 & 46.4 \\
\hline Domestic debt, net (end of period) & 24.3 & 26.8 & 22.2 & 22.3 & 22.5 & 21.7 & 20.3 \\
\hline NPV of external debt (end of period) & 27.6 & 28.8 & 27.4 & 27.3 & 27.1 & 26.8 & 26.1 \\
\hline
\end{tabular}

Sources: Kenyan authorities; and staff estimates and projections.

1/ 12-month period ended June 2003. 
Table 2. Kenya: Monetary Survey, Base Case Scenario, 2003-2007

\begin{tabular}{|c|c|c|c|c|c|c|c|c|c|c|c|c|c|c|c|}
\hline \multirow[b]{3}{*}{ Central Bank of Kenya (CBK) } & \multirow[t]{3}{*}{$\begin{array}{r}\text { Jun-03 } \\
\text { Actual } \\
\end{array}$} & \multicolumn{2}{|c|}{\begin{tabular}{cc}
\multicolumn{2}{c}{ Sep-03 } \\
rogram & Actual
\end{tabular}} & \multicolumn{2}{|c|}{$\begin{array}{c}\text { Dec-03 } \\
\text { gram Actual }\end{array}$} & \multicolumn{2}{|c|}{\begin{tabular}{cc}
\multicolumn{2}{c}{ Mar-04 } \\
am & Actual \\
\end{tabular}} & \multicolumn{2}{|c|}{$\begin{array}{cc}\text { Jun-04 } \\
\text { Program } & \text { Actual } \\
\end{array}$} & $\begin{array}{r}\text { Sep-04 } \\
\text { Actual } \\
\end{array}$ & \multirow[t]{2}{*}{ Dec-04 } & \multirow[t]{2}{*}{ Mar-05 } & \multirow[t]{2}{*}{ Jun-05 } & \multirow[t]{2}{*}{ Jun-06 } & \multirow[t]{2}{*}{ Jun-07 } \\
\hline & & & & Billions $\mathrm{c}$ & Kenya Sh & illlings) & & & & & & & & & \\
\hline & & & & & & & & & & & & & & & \\
\hline $\begin{array}{l}\text { Net foreign assets } 1 / \\
\text { In Millions of US \$ }\end{array}$ & $\begin{array}{r}81.5 \\
1,032\end{array}$ & $\begin{array}{r}81.5 \\
1,032\end{array}$ & $\begin{array}{r}84.1 \\
1,065\end{array}$ & $\begin{array}{r}83.1 \\
1,053\end{array}$ & $\begin{array}{r}88.3 \\
1,118\end{array}$ & $\begin{array}{r}89.4 \\
1,133\end{array}$ & $\begin{array}{r}85.3 \\
1,081\end{array}$ & $\begin{array}{r}95.6 \\
1,211\end{array}$ & $\begin{array}{r}86.7 \\
1.098\end{array}$ & $\begin{array}{r}79.8 \\
1.011\end{array}$ & $\begin{array}{r}79.6 \\
1009\end{array}$ & $\begin{array}{r}84.5 \\
1.070\end{array}$ & & $\begin{array}{r}98.9 \\
1.253\end{array}$ & $\begin{array}{l}103.0 \\
1.305\end{array}$ \\
\hline $\begin{array}{l}\text { In Millions of US \$ } \\
\text { Net domestic assets }\end{array}$ & $\begin{array}{r}1,032 \\
4.0\end{array}$ & -2.8 & $\begin{array}{r}1,063 \\
-2.3\end{array}$ & 0.5 & $\begin{array}{r}1,118 \\
-0.7\end{array}$ & $\begin{array}{r}1,133 \\
-8.7\end{array}$ & $\begin{array}{r}1,081 \\
1.3\end{array}$ & $\begin{array}{r}1,211 \\
-14.8\end{array}$ & $\begin{array}{r}1,098 \\
3.6\end{array}$ & $\begin{array}{r}1,011 \\
10.0\end{array}$ & $\begin{array}{r}1,009 \\
13.3\end{array}$ & $\begin{array}{r}1,070 \\
8.8\end{array}$ & $\begin{array}{r}1,212 \\
-2.0\end{array}$ & $\begin{array}{r}1,253 \\
1.1\end{array}$ & $\begin{array}{r}1,305 \\
4.0\end{array}$ \\
\hline $\begin{array}{l}\text { Net domestic assets } \\
\text { Net domestic credit }\end{array}$ & 7.7 & -1.6 & 0.5 & 1.8 & 1.8 & -7.3 & 7.2 & -13.3 & 11.2 & 14.3 & 17.6 & 13.3 & 2.7 & 6.1 & 9.2 \\
\hline Government (net) & 14.4 & 14.8 & -0.3 & 15.2 & 2.4 & 15.6 & 10.2 & 16.0 & 15.5 & 17.4 & 18.1 & 18.7 & 19.1 & 20.6 & 21.1 \\
\hline Private sector credit (CBK staff loans) & 1.7 & 1.7 & 1.8 & 1.7 & 1.8 & 1.7 & 1.8 & 1.8 & 1.9 & 1.9 & 1.9 & 1.9 & 2.0 & 2.1 & 2.1 \\
\hline Commercial banks (net REPOS) & -8.4 & -18.1 & -1.0 & -15.1 & -2.5 & -24.7 & -4.9 & -31.1 & -6.1 & -5.0 & -2.3 & -7.3 & -18.3 & -16.6 & -14.1 \\
\hline Other items (net) & -3.7 & -1.2 & -2.8 & -1.3 & -2.5 & -1.4 & -5.9 & -1.5 & -7.7 & -4.3 & -4.3 & -4.5 & -4.8 & -5.0 & -5.2 \\
\hline Reserve money (RM) & 85.5 & 78.7 & 81.8 & 83.6 & 87.5 & 80.7 & 86.6 & 80.9 & 90.2 & 89.9 & 93.0 & 93.3 & 93.7 & 100.1 & 107.1 \\
\hline Currency outside banks & 49.7 & 51.2 & 49.4 & 55.8 & 55.5 & 53.0 & 54.9 & 53.6 & 55.7 & 56.1 & 57.9 & 57.7 & 57.7 & 61.7 & 66.0 \\
\hline Bank reserves & 35.8 & 27.5 & 32.4 & 27.8 & 32.0 & 27.7 & 31.7 & 27.2 & 34.6 & 33.8 & 35.1 & 35.5 & 36.0 & 38.3 & 41.0 \\
\hline Deposits-banks (Kenya) & 30.3 & 21.0 & 26.1 & 21.0 & 24.3 & $\ldots$ & 25.2 & 27.2 & 27.6 & 26.5 & 27.8 & 28.2 & 28.6 & 30.5 & 32.6 \\
\hline Required Reserves $2 /$ & 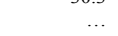 & $\ldots$ & 21.0 & $\cdots$ & 22.2 & $\ldots$ & 22.9 & $\cdots$ & 24.3 & 25.0 & 26.3 & 26.7 & 27.2 & 29.1 & 31.1 \\
\hline Excess Reserves & $\ldots$ & $\ldots$ & 5.1 & $\cdots$ & 2.1 & $\ldots$ & 2.3 & $\ldots$ & 3.3 & 1.6 & 1.6 & 1.5 & 1.5 & 1.4 & 1.5 \\
\hline Total till cash & 5.5 & $\ldots$ & 6.3 & $\ldots$ & 7.7 & $\ldots$ & 6.5 & $\ldots$ & 7.0 & 7.2 & 7.2 & 7.3 & 7.3 & 7.9 & 8.4 \\
\hline Banks & & & & & & & & & & & & & & & \\
\hline Net foreign assets $1 /$ & 22.9 & 22.6 & 23.4 & 22.4 & 23.8 & 22.2 & 36.8 & 22.0 & 33.1 & 37.2 & 37.1 & 35.3 & 33.5 & 27.3 & 22.2 \\
\hline $\begin{array}{l}\text { Reserves } \\
\text { Credit to CBK }\end{array}$ & 35.8 & 27.5 & 32.4 & 27.8 & 32.0 & 27.7 & 31.7 & 27.2 & 34.6 & 33.8 & 35.1 & 35.5 & 36.0 & 38.3 & 41.0 \\
\hline $\begin{array}{l}\text { Credit to CBK } \\
\text { Net domestic assets }\end{array}$ & 8.4 & 18.1 & 1.0 & 15.1 & 2.5 & 24.7 & 4.9 & 31.1 & 6.1 & 5.0 & 2.3 & 7.3 & 18.3 & 16.6 & 14.1 \\
\hline Net domestic assets & 302.6 & 305.2 & 318.5 & 308.1 & 339.5 & 310.7 & 332.3 & 315.0 & 343.9 & 354.5 & 361.4 & 365.5 & 363.3 & 400.1 & 438.0 \\
\hline $\begin{array}{l}\text { Domestic credit } \\
\text { Goverment (net) }\end{array}$ & 367.4 & 370.9 & 389.7 & 374.5 & 401.0 & 377.7 & 403.1 & 382.7 & 412.6 & 428.5 & 435.9 & 443.3 & 445.0 & 494.7 & 536.5 \\
\hline $\begin{array}{l}\text { Government (net) } \\
\text { Other public sector }\end{array}$ & 109.8 & 109.7 & 130.3 & 110.7 & 131.3 & 111.7 & 125.2 & 112.7 & 121.1 & 118.6 & 124.3 & 127.9 & 129.7 & 137.3 & 140.1 \\
\hline $\begin{array}{l}\text { Other public sector } \\
\text { Private sector }\end{array}$ & 6.3 & 10.0 & 6.0 & 8.3 & 6.0 & 7.8 & 8.7 & 6.5 & 9.2 & 11.3 & 6.2 & 9.0 & 9.5 & 9.9 & 10.2 \\
\hline $\begin{array}{l}\text { Private sector } \\
\text { Other items (net) }\end{array}$ & 251.3 & 251.2 & 253.4 & 255.5 & 263.7 & 258.3 & 269.3 & 263.5 & 282.3 & 298.5 & 305.4 & 306.4 & 305.7 & 347.5 & 386.2 \\
\hline Other items (net) & -64.8 & -65.7 & -71.2 & -66.4 & -61.4 & -67.0 & -70.8 & -67.7 & -68.7 & -74.0 & -74.4 & -77.8 & -81.7 & -94.6 & -98.4 \\
\hline Total deposits & 369.7 & 373.3 & 375.3 & 373.4 & 397.9 & 385.2 & 405.7 & 395.3 & 417.8 & 430.4 & 436.0 & 443.6 & 451.1 & 482.3 & 515.3 \\
\hline Monetary survey & & & & & & & & & & & & & & & \\
\hline Net foreign assets $1 /$ & 10 & 104.1 & 107.5 & 105.5 & 112.1 & 111.6 & 122.1 & 117.6 & 9.8 & 117.0 & 116.8 & 9.7 & 129.2 & 126.2 & 125.3 \\
\hline Net domestic assets & 315.1 & 320.9 & 317.2 & 326.4 & 341.2 & 326.9 & 338.5 & 331.7 & 353.6 & 369.5 & 377.1 & 381.5 & 379.6 & 417.8 & 456.1 \\
\hline Domestic & 383.6 & 387.4 & 391.2 & 391.4 & 405.2 & 395.0 & 415.2 & 400.5 & 430.0 & 447.8 & 455.9 & 3.9 & 466.0 & 17.3 & 559.7 \\
\hline Government (net) & 124.2 & 124.5 & 130.0 & 125.9 & 133.7 & 127.2 & 135.4 & 128.6 & 136.6 & 136.0 & 142.4 & 46.6 & 8.8 & 57.9 & 161.2 \\
\hline Rest of the economy & 259.3 & 262.9 & 261.2 & 265.5 & 271.5 & 267.8 & 279.8 & 271.9 & 293.4 & 311.8 & 313.5 & 317.3 & 317.2 & 359.5 & 398.5 \\
\hline Other public sector & $\begin{array}{r}6.3 \\
2530-x-2\end{array}$ & $\begin{array}{r}10.0 \\
0520\end{array}$ & $\begin{array}{r}6.0 \\
255\end{array}$ & $\begin{array}{r}8.3 \\
2572-1\end{array}$ & $\begin{array}{r}6.0 \\
265.5\end{array}$ & 7.8 & 8.7 & $\begin{array}{r}6.5 \\
2653 x-5\end{array}$ & 9.2 & $\begin{array}{r}11.3 \\
3005\end{array}$ & 6.2 & $\begin{array}{r}9.0 \\
308.3\end{array}$ & $\begin{array}{r}9.5 \\
3077\end{array}$ & $\begin{array}{r}9.9 \\
3496\end{array}$ & $\begin{array}{r}10.2 \\
338.3\end{array}$ \\
\hline $\begin{array}{l}\text { Private } \\
\text { Pats }\end{array}$ & 253.0 & 252.9 & 255.2 & 257.2 & 265.5 & 260.0 & 271.2 & 265.3 & 284.2 & 300.5 & 307.3 & 308.3 & 307.7 & 349.6 & 388.3 \\
\hline Other items (net) & -68.5 & -66.4 & -74.0 & -64.9 & -64.0 & -68.1 & -76.7 & -68.8 & -76.3 & -78.3 & -78.8 & -82.3 & -86.5 & -99.6 & -103.6 \\
\hline Money and quasi money ( & 362.6 & 368.8 & 370.3 & 376.3 & 395.1 & 383.4 & 394.8 & 394.7 & 407.3 & 417.0 & 424.3 & 431.8 & 439.3 & 474.7 & 512.2 \\
\hline $\mathrm{M} 3$ and for & 419.4 & 425.0 & 424.7 & 432.0 & 453.3 & 438.5 & 460.6 & 449.3 & 473.4 & 486.5 & 493.9 & 501.3 & 508.8 & 544.0 & 581.4 \\
\hline Currency outside banks & 49.7 & 51.2 & 49.4 & 55.8 & 55.5 & 53.0 & 54.9 & 53.6 & 55.7 & 56.1 & 57.9 & 57.7 & 57.7 & 61.7 & 66.0 \\
\hline 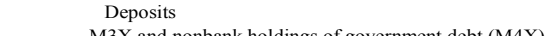 & 369.7 & 373.8 & 375.3 & 376.1 & 397.9 & 385.5 & 405.7 & 395.7 & 417.8 & 430.4 & 436.0 & 443.6 & 451.1 & 482.3 & 515.3 \\
\hline M3X and nonbank holdings of government debt (M4X) & 542.4 & 552.7 & 547.2 & 563.2 & 569.4 & 573.3 & 580.1 & 587.7 & 592.9 & 607.2 & 620.2 & 635.4 & 648.0 & 704.4 & 749.6 \\
\hline Memorandum items: & & & & & & & & & & & & & & & \\
\hline M3 & 93 & 9.8 & & 73 & $\begin{array}{l}\text { nual C } \\
127\end{array}$ & e) 8.7 & & 89 & 123 & 12.6 & 74 & 94 & 79 & 81 & \\
\hline M3X & $\begin{array}{r}9.3 \\
10.9\end{array}$ & 9.7 & $\begin{array}{r}10.3 \\
9.6\end{array}$ & 6.4 & $\begin{array}{ll}12.1 \\
11.7\end{array}$ & $\begin{array}{l}8.7 \\
7.3\end{array}$ & $\begin{array}{l}11.9 \\
12.7\end{array}$ & 7.1 & 12.9 & 14.5 & 8.9 & $\begin{array}{l}9.4 \\
8.8\end{array}$ & 7.5 & $\begin{array}{l}8.1 \\
6.9\end{array}$ & 6.9 \\
\hline $\mathrm{M} 4 \mathrm{X}$ & 12.1 & 10.7 & 9.6 & 8.1 & 9.3 & 8.7 & 10.0 & 8.3 & 9.3 & 11.0 & 8.9 & $\begin{array}{l}9.0 \\
9.5\end{array}$ & 9.3 & 8.7 & 6.4 \\
\hline Reserve Money & 11.2 & 0.1 & 4.1 & -5.5 & -1.1 & -4.1 & 3.0 & -5.4 & 5.5 & 9.8 & 6.2 & 7.7 & 3.8 & 6.8 & 7.0 \\
\hline Currency outside banks & 5.9 & 10.5 & 6.7 & 3.6 & 3.0 & 7.4 & 11.1 & 7.9 & 12.0 & 13.4 & 4.3 & 5.2 & 3.6 & 7.0 & 7.0 \\
\hline Net domestic asset: & 12.0 & 10.5 & 9.2 & 7.4 & 12.3 & 7.4 & 11.2 & 5.0 & 12.2 & 16. & 10.5 & 12.7 & 7.3 & 10.1 & 9.2 \\
\hline NDA growth (as percent of base period M3X) & 8.9 & 7.8 & 6.9 & 5.5 & 9.2 & 5.5 & 8.4 & 3.8 & 9.2 & 12.3 & 7.9 & 9.3 & 5.5 & 7.5 & 7.0 \\
\hline Other items net of banking sector & 5.5 & 5.1 & 14.0 & 14.8 & 6.3 & 6.1 & 12.1 & 5.1 & 6.0 & 3.9 & 21.2 & 9.9 & 19.0 & 15.8 & 4.1 \\
\hline Domes & 12. & 9.3 & 10 & 7.2 & 11 & 6.8 & 12. & 4.5 & 12. & 14. & 12 & 11.7 & 8.4 & 11 & 8.2 \\
\hline Government (net) & 31.1 & 22.5 & 27.9 & 15.9 & 23.1 & 10.8 & 17.9 & 3.9 & 9.9 & 4.6 & 6.5 & 8.3 & 8.9 & 6.1 & 2.1 \\
\hline Rest of the economy & 4.9 & 4.0 & 3.4 & 3.6 & 5.9 & 5.0 & 9.7 & 4.8 & 13.1 & 19.4 & 15.4 & 13.4 & 8.1 & 13.3 & 10.9 \\
\hline Non-bank holdings of gov & 123 & 128 & 123 & 131 & 116 & 135 & 120 & 138 & 119 & 121 & 126 & 134 & 139 & 160 & 168 \\
\hline Stock of domestic debt, billions of Ksh & 247 & 252 & 252 & 257 & 250 & 262 & 255 & 267 & 256 & 257 & 269 & 281 & 288 & 318 & 329 \\
\hline Multiplic & 4.9 & 5.4 & 5.2. & 5.2 & 5.2 & 5.4 & 5.3 & 5.6 & 5.2 & 5.4 & 5.3 & 5.4 & 5.4 & 5.4 & 5.4 \\
\hline Reserve cover, in months & 29 & 2.8 & 3.0 & 2.9 & 3.4 & 2.6 & 2.8 & 2.8 & 2.8 & 2.6 & 2.8 & 2.7 & 3.0 & .0 & 3.1 \\
\hline Bank Reserve/Total Deposit & 9.7 & 7.4 & 8.6 & 7.4 & 8.0 & 7.2 & 7.8 & 6.9 & 8.3 & 7.8 & 8.0 & 8.0 & 8.0 & 8.0 & 8.0 \\
\hline
\end{tabular}

Source: Central Bank of Kenya and staff projections

Note:

1/ Constant Kenya shilling per U.S. dollar exchange rate prevailing on September 30, 2001
2/ Calculated as $6 \%$ of total deposit 


\begin{tabular}{|c|c|c|c|c|c|c|c|c|c|c|}
\hline \multicolumn{11}{|c|}{ enario } \\
\hline & \multirow{2}{*}{$\frac{2001 / 02}{\text { Actual }}$} & \multirow{2}{*}{$\begin{array}{c}2002 / 03 \\
\text { Actual }\end{array}$} & \multirow{2}{*}{\multicolumn{2}{|c|}{ 2003/04 }} & \multicolumn{2}{|c|}{ 2004/05 } & \multicolumn{2}{|c|}{$2005 / 06$} & \multirow{2}{*}{$\begin{array}{c}\text { 2006/07 } \\
\text { Proj. }\end{array}$} & \multirow{2}{*}{$\begin{array}{c}2007 / 08 \\
\text { Proj. }\end{array}$} \\
\hline & & & & Prel. & Program & Proj. & $\begin{array}{c}\text { Program } \\
\text { Prat }\end{array}$ & Proj. & & \\
\hline Revenue & 197,768 & 210,750 & 239,177 & 252,681 & 259,660 & 277,809 & 285,205 & 300,371 & 336,471 & 378,001 \\
\hline Income tax & 59,034 & 70,452 & 71,565 & 81,402 & 74,377 & 92,398 & 79,485 & 97,608 & 107,310 & 118,358 \\
\hline Import duty (net) & 21,584 & 18,477 & 21,684 & 22,324 & 26,900 & 21,211 & 30,793 & 20,605 & 22,307 & 25,638 \\
\hline Excise duty & 32,077 & 35,643 & 39,499 & 40,085 & 43,465 & 43,853 & 47,746 & 45,104 & 50,407 & 55,410 \\
\hline Value-added tax & 50,871 & 56,135 & 57,124 & 61,725 & 67,209 & 73,324 & 73,677 & 77,467 & 84,978 & 93,670 \\
\hline Investment income & 767 & 1,243 & 1,868 & 3,331 & 1,980 & 3,175 & 2,133 & 3,474 & 3,742 & 4,031 \\
\hline Other & 14,585 & 12,349 & 40,522 & 19,603 & 36,601 & 22,075 & 39,080 & 22,791 & 25,250 & 27,849 \\
\hline Ministerial and Departmental Fees (AIA) & 18,850 & 16,451 & & 24,211 & & 21,772 & & 23,824 & 25,663 & 27,640 \\
\hline Revenue Measures 1/ & & & 6,915 & & 9,128 & 0 & 12,291 & 9,498 & 16,814 & 25,404 \\
\hline Expenditure and net lending & 228,980 & 265,947 & 299,321 & 272,705 & 333,563 & 331,695 & 363,497 & 406,716 & 441,803 & 476,034 \\
\hline Recurrent expenditure & 204,026 & 222,421 & 244,685 & 241,425 & 259,736 & 270,101 & 268,978 & 294,980 & 321,818 & 348,572 \\
\hline Interest payments & 30,384 & 36,026 & 33,742 & 29,700 & 34,936 & 38,361 & 35,357 & 39,849 & 42,503 & 45,579 \\
\hline $\begin{array}{l}\text { Domestic interest } \\
\text {. }\end{array}$ & 23,744 & $\begin{array}{l}27,567 \\
8459\end{array}$ & 27,323 & 23,281 & 27,596 & 30,700 & $\begin{array}{l}26,759 \\
8598\end{array}$ & $\begin{array}{l}34,097 \\
5752\end{array}$ & 36,309 & 37,028 \\
\hline Foreign interest due & 6,640 & 8,459 & 6,419 & 6,419 & 7,340 & 7,661 & 8,598 & 5,752 & 6,194 & 8,551 \\
\hline Wages and benefits (civil service) & 78,125 & 85,087 & 93,380 & 95,850 & 92,624 & 104,128 & 93,637 & 113,941 & 122,737 & 132,195 \\
\hline Civil service reform & 1,537 & 0 & 500 & 273 & 8,283 & 2,002 & 8,090 & 3,847 & 7,786 & 10,126 \\
\hline Pensions, etc. & 8,995 & 9,450 & 14,067 & 13,612 & 15,489 & 17,443 & 15,454 & 20,103 & 22,939 & 26,809 \\
\hline Other & 61,235 & 69,367 & 78,726 & 81,459 & 83,520 & 86,167 & 92,538 & 92,761 & 99,600 & 107,202 \\
\hline Of which: operations and maintenance & 39,157 & 44,101 & 53,549 & 55,757 & 59,050 & 53,702 & 66,950 & 64,007 & 72,117 & 81,109 \\
\hline Defense and NSIS & 21,980 & 22,933 & 24,270 & 20,203 & 24,884 & 21,000 & 23,903 & 22,979 & 24,753 & 26,660 \\
\hline Pending bills & 1,770 & -442 & 0 & 328 & 0 & 1,000 & 0 & 1,500 & 1,500 & 0 \\
\hline Drought relief expenditures & 0 & 0 & & 0 & 0 & 0 & & & & \\
\hline Development and net lending & 24,954 & 43,526 & 54,636 & 31,280 & 73,826 & 55,594 & 94,519 & 109,736 & 117,986 & 125,462 \\
\hline Domestically financed & 10,873 & 12,778 & 15,885 & 16,297 & 24,991 & 19,633 & 36,416 & 36,826 & 45,981 & 49,523 \\
\hline Foreign financed & 15,381 & 21,951 & 35,854 & 17,487 & 48,000 & 30,987 & 57,000 & 71,409 & 70,505 & 75,938 \\
\hline Net lending & 1,862 & 1,669 & 2,897 & 1,368 & 835 & 3,474 & 1,103 & 0 & 0 & 0 \\
\hline Pending bills & $-3,162$ & 7,128 & 0 & $-3,872$ & 0 & 1,000 & 0 & 1,500 & 1,500 & 0 \\
\hline Drought Development Expenditure & 0 & 0 & 0 & 0 & 0 & 500 & 然 & 0 & 0 & 0 \\
\hline Contingency $2 /$ & 0 & 0 & 0 & 0 & 0 & 6,000 & & 2,000 & 2,000 & 2,000 \\
\hline Balance (commitment basis, excluding grants) & $-31,212$ & $-55,197$ & $-60,144$ & $-20,024$ & $-73,902$ & $-53,886$ & $-78,291$ & $-106,345$ & $-105,332$ & $-98,033$ \\
\hline Grants & 6,823 & 14,942 & 25,051 & 16,224 & 25,000 & 20,828 & 30,000 & 57,022 & 51,841 & 50,540 \\
\hline Food relief grants & 0 & 0 & 0 & 0 & 0 & 0 & 0 & 0 & 0 & 0 \\
\hline Project grants & 5,350 & 14,484 & 21,102 & 11,841 & 25,000 & 16,078 & 30,000 & 52,149 & 46,924 & 50,540 \\
\hline Program grants & 1,473 & 458 & 3,949 & 4,383 & 0 & 4,750 & 0 & 4,873 & 4,916 & 0 \\
\hline Balance (commitment basis, including grants) & $-24,389$ & $-40,255$ & $-35,093$ & $-3,800$ & $-48,902$ & $-33,058$ & $-48,291$ & $-49,323$ & $-53,491$ & $-47,493$ \\
\hline Adjustments to cash basis & $-2,832$ & 7,163 & $-1,500$ & 1,470 & 0 & -348 & 0 & 0 & 0 & 0 \\
\hline Balance (cash basis, including grants) & $-27,221$ & $-33,092$ & $-36,593$ & $-2,330$ & $-48,902$ & $-33,406$ & $-48,291$ & $-49,323$ & $-53,491$ & $-47,493$ \\
\hline Financing & 26,621 & 32,101 & 7,580 & $-2,551$ & $-1,817$ & 33,406 & $-17,225$ & 44,240 & 32,489 & 11,806 \\
\hline Net foreign financing & $-11,250$ & $-10,340$ & $-12,234$ & $-8,860$ & 7,249 & 6,224 & 11,898 & 18,461 & 21,274 & 9,403 \\
\hline Project loans & 10,031 & 7,467 & 14,752 & 5,646 & 23,000 & 14,909 & 27,000 & 19,260 & 23,581 & 25,398 \\
\hline Program loans & 0 & 0 & 3,726 & 5,993 & 0 & 7,199 & 0 & 8,408 & 11,351 & 2,475 \\
\hline Financial defense lease loan adjustment & 2,064 & 1,803 & 2,515 & 0 & 2,259 & 0 & 373 & 0 & 0 & 0 \\
\hline Repayments due & $-21,951$ & $-17,428$ & $-19,429$ & $-24,794$ & $-15,397$ & $-24,814$ & $-15,475$ & $-18,752$ & $-18,208$ & $-18,470$ \\
\hline Change in arrears & $-1,394$ & $-2,182$ & $-13,798$ & 138 & $-2,613$ & $-2,245$ & 0 & 0 & 0 & 0 \\
\hline Rescheduling & 0 & 0 & 0 & 4,157 & 0 & 11,176 & 0 & 9,546 & 4,550 & 0 \\
\hline Privatization proceeds & 955 & 0 & 0 & 0 & 0 & 200 & 0 & 0 & 0 & 0 \\
\hline Bank restructuring costs & 0 & 0 & $-12,000$ & $-2,500$ & 0 & $-5,000$ & 0 & $-4,500$ & 0 & 0 \\
\hline Expenditure arrears securitization & $-2,788$ & $-4,481$ & $-3,667$ & 0 & $-3,667$ & 0 & $-3,667$ & 0 & 0 & 0 \\
\hline Net domestic borrowing & 39,704 & 46,922 & 35,481 & 8,809 & $-5,400$ & 31,982 & $-25,457$ & 30,279 & 11,216 & 2,403 \\
\hline Financing gap (stat. discrepancy for outturns) 3/ & 600 & 991 & 29,013 & 4,881 & 50,720 & 0 & 65,516 & 5,083 & 21,002 & 35,687 \\
\hline \multicolumn{11}{|l|}{ Memorandum items: } \\
\hline Total project support & 15,381 & 21,951 & 35,854 & 17,487 & 48,000 & 30,987 & 57,000 & 71,409 & 70,505 & 75,938 \\
\hline Total identified and unidentified gross external support & 16,854 & 23,400 & 72,542 & 32,744 & 98,720 & 42,936 & 122,516 & 89,772 & 107,774 & 114,100 \\
\hline Nominal GDP & 920,708 & $1,027,163$ & $1,076,174$ & $1,162,052$ & $1,140,956$ & $1,300,651$ & $1,229,134$ & $1,423,226$ & $1,533,090$ & $1,651,231$ \\
\hline Primary budget balance & 3,163 & 2,934 & $-2,851$ & 27,370 & $-13,966$ & 4,955 & $-12,935$ & $-9,473$ & $-10,988$ & $-1,914$ \\
\hline Stock of domestic debt, net (end of period) & 202,775 & 249,697 & 288,592 & 258,506 & 283,192 & 290,488 & 257,736 & 320,767 & 331,982 & 334,385 \\
\hline
\end{tabular}

Sources: Kenyan authorities; and Fund staff estimates and projections.

$1 /$ Revenue measures is a policy variable that reflects resources needed to achieve policy targets.

3 / Financing gap includes uncommitted external project and program grants and loas. 
Table 3b. Kenya: Central Government Financial Operations, 2001/02-2007/08

\begin{tabular}{|c|c|c|c|c|c|c|c|c|c|c|}
\hline & $2001 / 02$ & $2002 / 03$ & \multicolumn{2}{|c|}{ 2003/04 } & \multicolumn{2}{|c|}{$2004 / 05$} & \multicolumn{2}{|c|}{$2005 / 06$} & \multirow{2}{*}{$\begin{array}{c}2006 / 07 \\
\text { Proj. }\end{array}$} & \multirow{2}{*}{$\frac{2007 / 08}{\text { Proj. }}$} \\
\hline & Actual & $\begin{array}{l}\text { Actual } \\
\end{array}$ & Program & Prel. & Program & Proj. & $\begin{array}{l}\text { Program } \\
\end{array}$ & Proj. & & \\
\hline Revenue & 21.5 & 20.5 & 22.2 & 21.7 & 22.8 & 21.4 & 23.2 & 21.1 & 21.9 & 22.9 \\
\hline Income tax & 6.4 & 6.9 & 6.6 & 7.0 & 6.5 & 7.1 & 6.5 & 6.9 & 7.0 & 7.2 \\
\hline Import duty (net) & 2.3 & 1.8 & 2.0 & 1.9 & 2.4 & 1.6 & 2.5 & 1.4 & 1.5 & 1.6 \\
\hline Excise duty & 3.5 & 3.5 & 3.7 & 3.4 & 3.8 & 3.4 & 3.9 & 3.2 & 3.3 & 3.4 \\
\hline Value-added tax & 5.5 & 5.5 & 5.3 & 5.3 & 5.9 & 5.6 & 6.0 & 5.4 & 5.5 & 5.7 \\
\hline Investment income & 0.1 & 0.1 & 0.2 & 0.3 & 0.2 & 0.2 & 0.2 & 0.2 & 0.2 & 0.2 \\
\hline Other & 1.6 & 1.2 & 3.8 & 1.7 & 3.2 & 1.7 & 3.2 & 1.6 & 1.6 & 1.7 \\
\hline $\begin{array}{l}\text { Ministerial and Departmental Fees (AIA) } \\
\text { Revenue Measures 1/ }\end{array}$ & $\begin{array}{l}2.0 \\
0.0\end{array}$ & $\begin{array}{l}1.6 \\
0.0\end{array}$ & $\begin{array}{l}0.0 \\
0.6\end{array}$ & $\begin{array}{l}2.1 \\
0.0\end{array}$ & $\begin{array}{l}0.0 \\
0.8\end{array}$ & $\begin{array}{l}1.7 \\
0.0\end{array}$ & $\begin{array}{l}0.0 \\
1.0\end{array}$ & $\begin{array}{l}1.7 \\
0.7\end{array}$ & $\begin{array}{l}1.7 \\
1.1\end{array}$ & $\begin{array}{l}1.7 \\
1.5\end{array}$ \\
\hline Expenditure and net lending & 24.9 & 25.9 & 27.8 & 23.5 & 29.2 & 25.5 & 29.6 & 28.6 & 28.8 & 28.8 \\
\hline Recurrent expenditure & 22.2 & 21.7 & 22.7 & 20.8 & 22.8 & 20.8 & 21.9 & 20.7 & 21.0 & 21.1 \\
\hline Interest payments & 3.3 & 3.5 & 3.1 & 2.6 & 3.1 & 2.9 & 2.9 & 2.8 & 2.8 & 2.8 \\
\hline Domestic interest & 2.6 & 2.7 & 2.5 & 2.0 & 2.4 & 2.4 & 2.2 & 2.4 & 2.4 & 2.2 \\
\hline Foreign interest due & 0.7 & 0.8 & 0.6 & 0.6 & 0.6 & 0.6 & 0.7 & 0.4 & 0.4 & 0.5 \\
\hline Wages and benefits (civil service) & 8.5 & 8.3 & 8.7 & 8.2 & 8.1 & 8.0 & 7.6 & 8.0 & 8.0 & 8.0 \\
\hline Civil service reform & 0.2 & 0.0 & 0.0 & 0.0 & 0.7 & 0.2 & 0.7 & 0.3 & 0.5 & 0.6 \\
\hline Pensions, etc. & 1.0 & 0.9 & 1.3 & 1.2 & 1.4 & 1.3 & 1.3 & 1.4 & 1.5 & 1.6 \\
\hline Y. onerations and maintenance & 6.7 & 6.8 & 7.3 & 7.0 & 7.3 & 6.6 & 7.5 & 6.5 & 6.5 & 6.5 \\
\hline Of which: operations and maintenance & 4.3 & 4.3 & 5.0 & 4.8 & 5.2 & 4.1 & 5.4 & 4.5 & 4.7 & 4.9 \\
\hline Defense and NSIS & 2.4 & 2.2 & 2.3 & 1.7 & 2.2 & 1.6 & 1.9 & 1.6 & 1.6 & 1.6 \\
\hline Pending bills & 0.2 & 0.0 & 0.0 & 0.0 & 0.0 & 0.1 & 0.0 & 0.1 & 0.1 & 0.0 \\
\hline Drought relief expenditures & 0.0 & 0.0 & 0.0 & 0.0 & 0.0 & 0.0 & 0.0 & 0.0 & 0.0 & 0.0 \\
\hline Development and net lending & 2.7 & 4.2 & 5.1 & 2.7 & 6.5 & 4.3 & 7.7 & 7.7 & 7.7 & 7.6 \\
\hline Domestically financed & 1.2 & 1.2 & 1.5 & 1.4 & 2.2 & 1.5 & 3.0 & 2.6 & 3.0 & 3.0 \\
\hline Foreign financed & 1.7 & 2.1 & 3.3 & 1.5 & 4.2 & 2.4 & 4.6 & 5.0 & 4.6 & 4.6 \\
\hline Net lending & 0.2 & 0.2 & 0.3 & 0.1 & 0.1 & 0.3 & 0.1 & 0.0 & 0.0 & 0.0 \\
\hline Pending bills & -0.3 & 0.7 & 0.0 & -0.3 & 0.0 & 0.1 & 0.0 & 0.1 & 0.1 & 0.0 \\
\hline Drought Development Expenditure & 0.0 & 0.0 & 0.0 & 0.0 & 0.0 & 0.0 & 0.0 & 0.0 & 0.0 & 0.0 \\
\hline Contingency 2/ & 0.0 & 0.0 & 0.0 & 0.0 & 0.0 & 0.5 & 0.0 & 0.1 & 0.1 & 0.1 \\
\hline Balance (commitment basis, excluding grants) & -3.4 & -5.4 & -5.6 & -1.7 & -6.5 & -4.1 & -6.4 & -7.5 & -6.9 & -5.9 \\
\hline Grants & 0.7 & 1.5 & 2.3 & 1.4 & 2.2 & 1.6 & 2.4 & 4.0 & 3.4 & 3.1 \\
\hline Food relief grants & 0.0 & 0.0 & 0.0 & 0.0 & 0.0 & 0.0 & 0.0 & 0.0 & 0.0 & 0.0 \\
\hline Project grants & 0.6 & 1.4 & 2.0 & 1.0 & 2.2 & 1.2 & 2.4 & 3.7 & 3.1 & 3.1 \\
\hline Program grants & 0.2 & 0.0 & 0.4 & 0.4 & 0.0 & 0.4 & 0.0 & 0.3 & 0.3 & 0.0 \\
\hline Balance (commitment basis, including grants) & -2.6 & -3.9 & -3.3 & -0.3 & -4.3 & -2.5 & -3.9 & -3.5 & -3.5 & -2.9 \\
\hline Adjustments to cash basis & -0.3 & 0.7 & -0.1 & 0.1 & 0.0 & 0.0 & 0.0 & 0.0 & 0.0 & 0.0 \\
\hline Balance (cash basis, including grants) & -3.0 & -3.2 & -3.4 & -0.2 & -4.3 & -2.6 & -3.9 & -3.5 & -3.5 & -2.9 \\
\hline Financing & 2.9 & 3.1 & 0.7 & -0.2 & -0.2 & 2.6 & -1.4 & 3.1 & 2.1 & 0.7 \\
\hline Net foreign financing & -1.2 & -1.0 & -1.1 & -0.8 & 0.6 & 0.5 & 1.0 & 1.3 & 1.4 & 0.6 \\
\hline Project loans & 1.1 & 0.7 & 1.4 & 0.5 & 2.0 & 1.1 & 2.2 & 1.4 & 1.5 & 1.5 \\
\hline Program loans & 0.0 & 0.0 & 0.3 & 0.5 & 0.0 & 0.6 & 0.0 & 0.6 & 0.7 & 0.1 \\
\hline Financial defense lease loan adjustment & 0.2 & 0.2 & 0.2 & 0.0 & 0.2 & 0.0 & 0.0 & 0.0 & 0.0 & 0.0 \\
\hline Repayments due & -2.4 & -1.7 & -1.8 & -2.1 & -1.3 & -1.9 & -1.3 & -1.3 & -1.2 & -1.1 \\
\hline Change in arrears & -0.2 & -0.2 & -1.3 & 0.0 & -0.2 & -0.2 & 0.0 & 0.0 & 0.0 & 0.0 \\
\hline Rescheduling & 0.0 & 0.0 & 0.0 & 0.4 & 0.0 & 0.9 & 0.0 & 0.7 & 0.3 & 0.0 \\
\hline Privatization proceeds & 0.1 & 0.0 & 0.0 & 0.0 & 0.0 & 0.0 & 0.0 & 0.0 & 0.0 & 0.0 \\
\hline Bank restructuring costs & 0.0 & 0.0 & -1.1 & -0.2 & 0.0 & -0.4 & 0.0 & -0.3 & 0.0 & 0.0 \\
\hline Expenditure arrears securitization & -0.3 & -0.4 & -0.3 & 0.0 & -0.3 & 0.0 & -0.3 & 0.0 & 0.0 & 0.0 \\
\hline Net domestic borrowing & 4.3 & 4.6 & 3.3 & 0.8 & -0.5 & 2.5 & -2.1 & 2.1 & 0.7 & 0.1 \\
\hline Financing gap (stat. discrepancy for outturns) 3/ & 0.1 & 0.1 & 2.7 & 0.4 & 4.4 & 0.0 & 5.3 & 0.4 & 1.4 & 2.2 \\
\hline \multicolumn{11}{|l|}{ Memorandum items: } \\
\hline Total project support & 1.7 & 2.1 & 3.3 & 1.5 & 4.2 & 2.4 & 4.6 & 5.0 & 4.6 & 4.6 \\
\hline Total identified and unidentified gross external support & 1.8 & 2.3 & 6.7 & 2.8 & 8.7 & 3.3 & 10.0 & 6.3 & 7.0 & 6.9 \\
\hline Nominal GDP & 920,708 & $1,027,163$ & $1,076,174$ & $1,162,052$ & $1,140,956$ & $1,300,651$ & $1,229,134$ & $1,423,226$ & $1,533,090$ & $1,651,231$ \\
\hline Primary budget balance & 0.3 & 0.3 & -0.3 & 2.4 & -1.2 & 0.4 & -1.1 & -0.7 & -0.7 & -0.1 \\
\hline Stock of domestic debt, net (end of period) & 22.0 & 24.3 & 26.8 & 22.2 & 24.8 & 22.3 & 21.0 & 22.5 & 21.7 & 20.3 \\
\hline
\end{tabular}

Sources: Kenyan authorities; and Fund staff estimates and projections.

1/ Revenue measures is a policy variable that reflects resources needed to achieve policy targets.

2/ Includes allocation of KSh $4 \mathrm{bln}$ to meet costs of additional emergency food supply. 
Table 4. Kenya: Balance of Payments, 2001/02-2007/08 1/

(In millions of U.S. dollars, unless otherwise indicated)

\begin{tabular}{|c|c|c|c|c|c|c|c|}
\hline & \multirow[b]{2}{*}{$2001 / 02$} & \multirow[b]{2}{*}{$2002 / 03$} & \multirow[b]{2}{*}{$\begin{array}{l}\text { 2003/04 } \\
\text { Prelim. }\end{array}$} & \multicolumn{3}{|c|}{ Program } & \multirow[b]{2}{*}{$\begin{array}{c}2007 / 08 \\
\text { Proj. }\end{array}$} \\
\hline & & & & $\begin{array}{c}\text { 2004/05 } \\
\text { Proj. }\end{array}$ & $\begin{array}{c}2005 / 06 \\
\text { Proj. }\end{array}$ & $\begin{array}{c}2006 / 07 \\
\text { Proj. }\end{array}$ & \\
\hline Current account & -62 & 144 & -219 & -704 & -970 & -949 & -813 \\
\hline Excluding official transfers & -86 & 144 & -282 & -764 & $-1,030$ & $-1,009$ & -813 \\
\hline Exports, f.o.b. & 1,962 & 2,322 & 2,491 & 2,807 & 3,068 & 3,324 & 3,661 \\
\hline Coffee & 85 & 85 & 82 & 91 & 102 & 115 & 133 \\
\hline Tea & 421 & 433 & 450 & 477 & 518 & 585 & 661 \\
\hline Horticulture & 241 & 296 & 371 & 408 & 459 & 523 & 594 \\
\hline Imports, f.o.b. & $-2,823$ & $-3,215$ & $-3,870$ & $-4,726$ & $-5,139$ & $-5,363$ & $-5,730$ \\
\hline Balance on goods & -860 & -893 & $-1,379$ & $-1,918$ & $-2,072$ & $-2,039$ & $-2,069$ \\
\hline Services (net) & 274 & 497 & 490 & 491 & 401 & 394 & 591 \\
\hline Credit & 1,060 & 1,169 & 1,198 & 1,361 & 1,509 & 1,644 & 1,757 \\
\hline Foreign travel & 277 & 330 & 372 & 459 & 567 & 664 & 730 \\
\hline Debit & -786 & -672 & -708 & -870 & $-1,108$ & $-1,250$ & $-1,166$ \\
\hline Balance on goods and services & -587 & -396 & -889 & $-1,427$ & $-1,670$ & $-1,645$ & $-1,478$ \\
\hline Income (net) & -166 & -104 & -152 & -121 & -126 & -133 & -134 \\
\hline Credit & 39 & 56 & 50 & 60 & 65 & 68 & 72 \\
\hline Debit & -205 & -160 & -203 & -180 & -191 & -202 & -206 \\
\hline Of which: official interest payments & -107 & -102 & -114 & -94 & -98 & -101 & -104 \\
\hline Other & -98 & -58 & -88 & -86 & -93 & -101 & -102 \\
\hline Current transfers (net) & 690 & 644 & 821 & 845 & 826 & 830 & 799 \\
\hline Private (net) & 667 & 644 & 759 & 785 & 766 & 770 & 799 \\
\hline Official (net) & 23 & 0 & 63 & 60 & 60 & 60 & 0 \\
\hline Capital and financial account & 160 & 88 & 460 & 671 & 878 & 937 & 935 \\
\hline Capital account & 70 & 192 & 271 & 266 & 276 & 313 & 285 \\
\hline Of which: capital transfers & 70 & 192 & 271 & 314 & 277 & 284 & 285 \\
\hline Financial account & 91 & -104 & 189 & 358 & 602 & 652 & 650 \\
\hline Investment assets and liabilities (net) & -38 & 116 & -80 & 388 & 431 & 550 & 587 \\
\hline Official, medium and long term & -124 & -56 & 58 & 187 & 195 & 258 & 204 \\
\hline Inflows & 170 & 153 & 332 & 425 & 431 & 498 & 458 \\
\hline Program loans & 0 & 0 & 79 & 96 & 104 & 114 & 30 \\
\hline Project loans & 128 & 97 & 191 & 255 & 245 & 288 & 331 \\
\hline Defence loans & 0 & 0 & 33 & 27 & 21 & 21 & 21 \\
\hline Government guaranteed/parastatal & 42 & 56 & 30 & 48 & 61 & 76 & 76 \\
\hline Outflows & -294 & -209 & -274 & -238 & -236 & -240 & -254 \\
\hline Commercial banks (net) & 60 & -7 & -181 & 50 & 40 & 13 & 0 \\
\hline Private (net) & 25 & 179 & 43 & 151 & 195 & 280 & 383 \\
\hline Short-term (net) and net errors and omissions $2 /$ & 129 & -220 & 269 & -31 & 171 & 102 & 62 \\
\hline Overall balance & 98 & 232 & 240 & -32 & -91 & -13 & 122 \\
\hline Financing items 3 / & -98 & -232 & -240 & 32 & 92 & 13 & -122 \\
\hline Reserve assets (gross) & -182 & -124 & -139 & -230 & -94 & -106 & -113 \\
\hline Use of Fund credit and loans to the Fund (net) & -24 & -19 & 20 & 134 & 67 & 63 & -9 \\
\hline Change in arrears & 48 & -90 & -170 & 0 & 0 & 0 & 0 \\
\hline Rescheduling & 61 & 0 & 48 & 129 & 118 & 56 & 0 \\
\hline Remaining gap & 0 & 0 & 0 & 0 & 0 & 0 & 0 \\
\hline Tentatively identified program support & 0 & 0 & 0 & 0 & 0 & 0 & 0 \\
\hline Unidentified support & 0 & 0 & 0 & 0 & 0 & 0 & 0 \\
\hline \multicolumn{8}{|l|}{ Memorandum items: } \\
\hline Gross official reserves (end of period) & 1,137 & 1,260 & 1,399 & 1,629 & 1,723 & 1,829 & 1,942 \\
\hline (in months of next year's imports of goods and services) & 3.5 & 3.3 & 3.0 & 3.1 & 3.1 & 3.2 & 3.1 \\
\hline (In months of this year's imports of goods and services) & 3.8 & 3.9 & 3.7 & 3.5 & 3.3 & 3.3 & 3.4 \\
\hline Stock of arrears & 0 & 280 & 56 & 0 & 0 & 0 & 0 \\
\hline Current account balance (excluding official transfers) & $-0.8 \%$ & $1.2 \%$ & $-2.0 \%$ & $-4.9 \%$ & $-6.1 \%$ & $-5.6 \%$ & $-4.2 \%$ \\
\hline
\end{tabular}

Sources: Kenyan authorities; and staff estimates and projections.

1/ This table assumes that Kenya's PRGF program is augmented by SDR 50 million, as requested by the authorities. Without the augmentation, there would be a financing gap of US\$ 74 million in FY 2004/05.

2/ This entry is believed to include underrecorded tourism earnings.

3/ Projections under the first-year program for fiscal year 2004/05 are not available because a switch from a calendar year to a fiscal year BOP took place since the November 2003 staff report, which provided only a calendar year BOP. 
Table 5. Kenya: Financial Soundness Indicators for the Banking Sector, 1999-2004 (In percent, unless otherwise indicated)

\begin{tabular}{|c|c|c|c|c|c|c|}
\hline & Dec-99 & Dec-00 & Dec-01 & Dec-02 & Dec-03 & Jun-04 \\
\hline \multicolumn{7}{|l|}{ Capital Adequacy } \\
\hline Regulatory capital to risk-weighted assets & $\ldots$. & 17.6 & 17.3 & 17.0 & 17.3 & 16.5 \\
\hline Regulatory Tier I capital to risk-weighted assets & & 14.2 & 14.5 & 14.1 & 16.3 & 15.6 \\
\hline Capital (net worth) to assets & 11.4 & 12.9 & 13.3 & 11.6 & 11.8 & 11.4 \\
\hline \multicolumn{7}{|l|}{ Asset composition and quality } \\
\hline \multicolumn{7}{|l|}{ Sectoral distribution of loans (percent of total loans) } \\
\hline Public sector & 3.9 & 3.9 & 3.7 & 4.2 & 4.2 & 4.0 \\
\hline Agriculture & 10.2 & 10.0 & 10.0 & 9.8 & 9.5 & 8.0 \\
\hline Manufacturing & 21.1 & 21.9 & 19.1 & 18.1 & 17.7 & 16.0 \\
\hline Building and construction & 7.1 & 6.2 & 6.6 & 6.3 & 5.7 & 6.0 \\
\hline Transport and communication & 3.7 & 3.5 & 3.8 & 6.0 & 5.7 & 6.0 \\
\hline Trade & 15.2 & 15.3 & 14.7 & 14.7 & 15.7 & 15.0 \\
\hline Other & 38.8 & 39.2 & 42.1 & 40.9 & 41.5 & 45.0 \\
\hline Nonperforn & $\ldots$. & 33.3 & 30.1 & 29.8 & 25.6 & 22.9 \\
\hline Nonperforming loa & $\ldots$. & 78.7 & 78.8 & 77.8 & 60.7 & 52.9 \\
\hline Foreign currency (FX) loans to total loans & 2.2 & 12.5 & 14.9 & 15.4 & 15.8 & 14.9 \\
\hline Spread between highest and lowest interbank rates & 19.3 & 3.5 & 3.0 & 2.0 & 0.8 & 0.3 \\
\hline \multicolumn{7}{|l|}{ Earnings and Profitability } \\
\hline Return on average assets (before tax) & 0.7 & 0.5 & 1.6 & 1.0 & 2.3 & 2.1 \\
\hline Retı & 7.9 & 4.9 & 15.7 & 10.9 & 23.2 & 22.7 \\
\hline Intere & 48.8 & 42.1 & 43.6 & 43.8 & 50.5 & 50.0 \\
\hline Nonin & 61.8 & 65.4 & 58.6 & 66.9 & 62.9 & 64.9 \\
\hline Personnel expense & 36.6 & 35.3 & 37.6 & 38.1 & 37.0 & 40.1 \\
\hline Trading and fee income to total income & 18.7 & 27.6 & 30.2 & 34.3 & 37.4 & 38.8 \\
\hline Spread between average lending and deposit rates & $\ldots$. & 13.4 & 13.8 & 13.6 & 10.2 & 10.0 \\
\hline \multicolumn{7}{|l|}{ Liquidity } \\
\hline Liquid assets & 28.4 & 29.5 & 34.4 & 33.7 & 33.2 & 35.7 \\
\hline Liquid assets to total short-te & 38.3 & 38.9 & 43.7 & 42.6 & 48.9 & 46.3 \\
\hline Custo & 111.0 & 113.7 & 121.2 & 125.2 & 131.6 & 139.1 \\
\hline Foreign currency liabilit & 6.3 & 16.2 & 16.0 & 16.2 & 14.3 & 15.8 \\
\hline \multicolumn{7}{|l|}{ Sensitivity to market risk } \\
\hline Net open positions in FX to capital & & . & 14.0 & 23.0 & 12.0 & 11.7 \\
\hline Net open positions in equities to capital & 9.7 & 9.3 & 11.4 & 12.8 & 7.9 & 7.9 \\
\hline
\end{tabular}

Source: Central Bank of Kenya and staff calculations 
Table 6: Kenya. Disbursement Schedule Under the Three-Year PRGF Arrangement

\begin{tabular}{|c|c|c|c|}
\hline Disbursement & $\begin{array}{c}\text { Amount } \\
\text { (In millions of SDRs) }\end{array}$ & Date & $\begin{array}{l}\text { Contingent on } \\
\text { Board Approval }\end{array}$ \\
\hline $1 \mathrm{st}$ & 25 & November 2003 & November 2003 \\
\hline 2nd & 50 & December 2004 & $\begin{array}{l}\text { end-December } 2003 \text { performance } \\
\text { criteria (PC) }\end{array}$ \\
\hline $3 \mathrm{rd}$ & 50 & July 1, 2005 & $\begin{array}{l}\text { end-March } 2005 \text { PC, and completion } \\
\text { of the second review by end- } \\
\text { June } 2005\end{array}$ \\
\hline 4 th & 25 & January 1, 2006 & $\begin{array}{l}\text { end-September } 2005 \mathrm{PC} \text {, and } \\
\text { completion of the third review by } \\
\text { end-December } 2005\end{array}$ \\
\hline 5 th & 25 & July 1, 2006 & $\begin{array}{l}\text { end-March } 2006 \text { PC, and completion } \\
\text { of the fourth review by end- } \\
\text { June } 2006\end{array}$ \\
\hline $6^{\text {th1 }}$ & 25 & January 1, 2007 & $\begin{array}{l}\text { end-September } 2006 \text { PC, and } \\
\text { completion of the fifth review by } \\
\text { end-December } 2006\end{array}$ \\
\hline 7 th $^{1}$ & 25 & July 1, 2007 & $\begin{array}{l}\text { end-March } 2007 \text { PC, and completion } \\
\text { of the sixth review by end-June } 2007\end{array}$ \\
\hline Total & 225 & & \\
\hline
\end{tabular}

1 While these disbursements at present fall outside the period of the arrangement, it is expected that the authorities will in the future request an extension of the arrangement. 
Table 7. Kenya: Macroeconomic Alternative Scenarios 1/

\begin{tabular}{|c|c|c|c|c|c|}
\hline & $2003 / 04$ & $2004 / 05$ & $2005 / 06$ & $2006 / 07$ & $2007 / 08$ \\
\hline & Prelim. & Proj. & Proj. & Proj. & Proj, \\
\hline (In percent of GDP, & ise indicat & ed) & & & \\
\hline A) & & & & & \\
\hline Overall & & & & & \\
\hline GDP volume (annual percentage change) & 2.1 & 2.7 & 3.2 & 3.6 & 4.0 \\
\hline Central government budget & & & & & \\
\hline Total revenue & 21.7 & 21.4 & 21.1 & 21.9 & 22.9 \\
\hline Total expenditure and net lending & 23.5 & 25.5 & 28.6 & 28.8 & 28.8 \\
\hline Overall balance (commitment basis) excluding grants & -1.7 & -4.1 & -7.5 & -6.9 & -5.9 \\
\hline Net domestic borrowing & 0.8 & 2.5 & 2.1 & 0.7 & 0.1 \\
\hline Financing gap & 0.4 & 0.0 & 0.4 & 1.4 & 2.2 \\
\hline Total donor support (grants \& loans) & 2.4 & 3.3 & 6.0 & 5.7 & 4.7 \\
\hline Balance of payments & & & & & \\
\hline Current external balance, excluding official transfers & -2.0 & -4.9 & -6.1 & -5.6 & -4.2 \\
\hline Gross international reserve coverage & & & & & \\
\hline in months of next year imports (end of period) & 3.0 & 3.1 & 3.1 & 3.2 & 3.1 \\
\hline Public Debt & & & & & \\
\hline NPV of central government debt (end of period) & 49.6 & 49.6 & 49.6 & 48.5 & 46.4 \\
\hline Domestic debt, net (end of period) & 22.2 & 22.3 & 22.5 & 21.7 & 20.3 \\
\hline NPV of external debt (end of period) & 27.4 & 27.3 & 27.1 & 26.8 & 26.1 \\
\hline B) & & & & & \\
\hline Overall & & & & & \\
\hline GDP volume (annual percentage change) & 2.1 & 3.5 & 4.3 & 5.0 & 5.5 \\
\hline Central government budget & & & & & \\
\hline Total revenue & 21.7 & 22.0 & 21.5 & 22.5 & 23.5 \\
\hline Total expenditure and net lending & 23.5 & 25.6 & 28.8 & 28.9 & 29.0 \\
\hline Overall balance (commitment basis) excluding grants & -1.7 & -3.6 & -7.3 & -6.4 & -5.5 \\
\hline Net domestic borrowing & 0.8 & 2.4 & 0.4 & -0.8 & -1.3 \\
\hline Financing gap & 0.4 & 0.0 & 0.4 & 1.4 & 2.2 \\
\hline Total donor support (grants \& loans) & 2.4 & 3.3 & 7.7 & 7.4 & 6.1 \\
\hline Balance of payments & & & & & \\
\hline Current external balance, excluding official transfers & -2.0 & -5.8 & -8.3 & -7.9 & -5.6 \\
\hline Gross international reserve coverage & & & & & \\
\hline in months of next year imports (end of period) & 3.0 & 3.3 & 4.0 & 4.1 & 4.5 \\
\hline Public Debt & & & & & \\
\hline NPV of central government debt (end of period) & 49.6 & 48.1 & 45.7 & 42.4 & 38.6 \\
\hline Domestic debt, net (end of period) & 22.2 & 22.1 & 20.4 & 17.9 & 15.1 \\
\hline NPV of external debt (end of period) & 27.4 & 26.0 & 25.3 & 24.5 & 23.5 \\
\hline C) & & & & & \\
\hline Overall & & & & & \\
\hline GDP volume (annual percentage change) & 2.1 & 1.7 & 2.2 & 2.5 & 2.9 \\
\hline Central government budget & & & & & \\
\hline Total revenue & 21.7 & 20.4 & 20.8 & 21.1 & 21.2 \\
\hline Total expenditure and net lending & 23.5 & 25.1 & 27.7 & 28.1 & 28.0 \\
\hline Overall balance (commitment basis) excluding grants & -1.7 & -4.7 & -6.9 & -7.0 & -6.8 \\
\hline Net domestic borrowing & 0.8 & 2.8 & 2.7 & 2.0 & 1.5 \\
\hline Financing gap & 0.4 & 0.0 & 0.4 & 1.4 & 2.2 \\
\hline Total donor support (grants \& loans) & 2.4 & 3.3 & 3.9 & 3.5 & 3.5 \\
\hline Balance of payments & & & & & \\
\hline Current external balance, excluding official transfers & -2.0 & -4.6 & -5.0 & -4.2 & -3.7 \\
\hline Gross international reserve coverage & & & & & \\
\hline in months of next year imports (end of period) & 3.0 & 2.8 & 2.7 & 2.5 & 2.5 \\
\hline Public Debt & & & & & \\
\hline NPV of central government debt (end of period) & 49.6 & 52.4 & 53.7 & 54.2 & 54.8 \\
\hline Domestic debt, net (end of period) & 22.2 & 22.6 & 23.6 & 24.1 & 25.1 \\
\hline NPV of external debt (end of period) & 27.4 & 29.8 & 30.1 & 30.1 & 29.7 \\
\hline
\end{tabular}

1/ The fiscal financing gap is set to be the same across all scenarios, with the net domestic borrowing treated as a residual. 
Table 8. Kenya: External Financing Requirements and Resources, 2001/02 - 2007/08

\begin{tabular}{|c|c|c|c|c|c|c|}
\hline & $2001 / 02$ & $2002 / 03$ & $2003 / 04$ & $2004 / 05$ & $2005 / 06$ & $2006 / 07$ \\
\hline External financing requirements & -538.8 & -297.3 & -880.5 & -1245.0 & -1365.4 & -1366.8 \\
\hline Current account (excl.official transfers) & -86.5 & 144.2 & -282.3 & -763.7 & -1030.0 & -1009.3 \\
\hline IMF payments, gross & -24.2 & -18.6 & -15.6 & -12.9 & -6.3 & -10.8 \\
\hline Change in arrears, net & 47.9 & -90.2 & -169.9 & 0.0 & 0.0 & 0.0 \\
\hline Buildup of gross official reserves & -182.0 & -123.6 & -138.7 & -230.0 & -93.6 & -106.2 \\
\hline Program support (committed and tentatively identified) & 23.4 & 0.0 & 177.3 & 302.4 & 237.0 & 247.5 \\
\hline IMF & 0.0 & 0.0 & 35.7 & 146.4 & 73.5 & 74.0 \\
\hline Program loans & 0.0 & 0.0 & 78.8 & 96.0 & 103.5 & 113.5 \\
\hline African Development Bank (ADB) & 0.0 & 0.0 & 21.0 & 21.0 & 33.5 & 33.5 \\
\hline IDA & 0.0 & 0.0 & 57.8 & 75.0 & 70.0 & 80.0 \\
\hline Program grants & 23.4 & 0.0 & 62.8 & 60.0 & 60.0 & 60.0 \\
\hline Project Support & 197.5 & 288.9 & 461.8 & 568.5 & 522.2 & 572.2 \\
\hline Project loans & 127.7 & 97.1 & 190.5 & 254.9 & 245.4 & 287.8 \\
\hline Private financing (incl. short-term (net) and errors \& omissions) & 214.6 & -47.4 & 130.5 & 170.8 & 406.4 & 394.7 \\
\hline Private financing, net & 85.6 & 172.6 & -138.4 & 201.4 & 235.6 & 292.7 \\
\hline of which, foreign direct investment & 25.8 & 80.3 & 25.0 & 106.0 & 170.0 & 280.0 \\
\hline Short-term (net) and net errors and omissions & 129.0 & -220.0 & 269.0 & -30.7 & 170.8 & 102.0 \\
\hline Rescheduling of debt & 61.0 & 0.0 & 48.0 & 128.8 & 118.1 & 55.5 \\
\hline Unidentified gap & 0.0 & 0.0 & 0.0 & 0.0 & -0.2 & 0.0 \\
\hline
\end{tabular}

Sources: Kenyan authorities and staff estimates and projections 
Table 9. Kenya: External Debt Indicators, 2001-2008 1/

(In millions of U.S. dollars, unless otherwise indicated)

\begin{tabular}{|c|c|c|c|c|c|c|}
\hline & 2003 & 2004 & 2005 & 2006 & 2007 & 2008 \\
\hline \multicolumn{7}{|l|}{ Debt-stock indicators } \\
\hline Stock of external debt by creditor $2 /$ & 5,117 & 5,250 & 5,562 & 5,854 & 6,128 & 6,324 \\
\hline Multilateral creditors & 3,159 & 3,264 & 3,558 & 3,783 & 3,953 & 4,058 \\
\hline IMF & 104 & 165 & 268 & 329 & 357 & 348 \\
\hline World Bank & 2,545 & 2,544 & 2,683 & 2,745 & 2,826 & 2,882 \\
\hline African Development Bank/African Development Fund & 320 & 351 & 387 & 469 & 491 & 513 \\
\hline Other & 191 & 203 & 221 & 240 & 279 & 315 \\
\hline Bilateral creditors & 1,631 & 1,690 & 1,762 & 1,868 & 2,005 & 2,125 \\
\hline Paris Club & 1,551 & 1,593 & 1,645 & 1,730 & 1,839 & 1,934 \\
\hline Non-Paris Club & 81 & 97 & 117 & 138 & 166 & 190 \\
\hline Other creditors & 326 & 296 & 242 & 203 & 170 & 141 \\
\hline Stock of external debt by debtor $2 /$ & 5,117 & 5,250 & 5,562 & 5,854 & 6,128 & 6,324 \\
\hline Central government & 4,691 & 4,739 & 4,923 & 5,096 & 5,288 & 5,439 \\
\hline Government guaranteed & 321 & 346 & 371 & 428 & 483 & 537 \\
\hline Central bank & 104 & 165 & 268 & 329 & 357 & 348 \\
\hline \multicolumn{7}{|l|}{ Net present value (NPV) of debt 3 / } \\
\hline In million of U.S dollars & 3,972 & 4,287 & 4,597 & 4,912 & 5,152 & 5,318 \\
\hline In percent of exports of goods and services 4 / & 111 & 109 & 105 & 103 & 100 & 94 \\
\hline In percent of government revenue, excluding grants & 132 & 129 & 130 & 126 & 119 & 112 \\
\hline In percent of GDP & 28 & 27 & 27 & 27 & 27 & 26 \\
\hline Stock of arrears & 56 & - & - & - & - & - \\
\hline \multicolumn{7}{|l|}{ Debt-service indicators 5/ } \\
\hline Principal payments by creditor & 323 & 258 & 262 & 260 & 248 & 262 \\
\hline Multilateral creditors & 113 & 108 & 100 & 112 & 104 & 108 \\
\hline IMF & 10 & 14 & 7 & 14 & 10 & 10 \\
\hline World Bank & 66 & 64 & 62 & 67 & 71 & 73 \\
\hline $\mathrm{AfDB} / \mathrm{AfDF}$ & 12 & 11 & 11 & 11 & 12 & 11 \\
\hline Other & 25 & 20 & 20 & 20 & 12 & 13 \\
\hline Bilateral creditors & 104 & 87 & 87 & 88 & 91 & 105 \\
\hline Paris Club & 97 & 84 & 85 & 85 & 88 & 100 \\
\hline Non-Paris Club & 7 & 3 & 3 & 3 & 3 & 5 \\
\hline Other creditors & 106 & 62 & 75 & 60 & 54 & 49 \\
\hline Interest payments by creditor & 92 & 94 & 98 & 87 & 107 & 111 \\
\hline Multilateral creditors & 32 & 32 & 35 & 37 & 39 & 41 \\
\hline IMF & 2 & 2 & 2 & 3 & 3 & 3 \\
\hline World Bank & 20 & 19 & 20 & 21 & 21 & 22 \\
\hline $\mathrm{AfDB} / \mathrm{AfDF}$ & 5 & 5 & 5 & 5 & 5 & 5 \\
\hline Other & 5 & 6 & 7 & 8 & 10 & 11 \\
\hline Bilateral creditors & 45 & 47 & 50 & 48 & 58 & 62 \\
\hline Paris Club & 40 & 38 & 36 & 32 & 33 & 31 \\
\hline Non-Paris Club & 5 & 9 & 14 & 16 & 25 & 31 \\
\hline Other creditors & 15 & 14 & 13 & 2 & 10 & 8 \\
\hline \multicolumn{7}{|l|}{ Debt-Service Ratios } \\
\hline \multicolumn{7}{|l|}{ Before 2004 Paris Club Rescheduling } \\
\hline Debt-service in percent of current year exports & $12 \%$ & $9 \%$ & $8 \%$ & $8 \%$ & $7 \%$ & $7 \%$ \\
\hline Debt-service in percent of fiscal revenue & $14 \%$ & $10 \%$ & $10 \%$ & $9 \%$ & $8 \%$ & $8 \%$ \\
\hline \multicolumn{7}{|l|}{ After 2004 Paris Club Rescheduling } \\
\hline Debt-service in percent of current year exports & - & $5 \%$ & $6 \%$ & $6 \%$ & $7 \%$ & $7 \%$ \\
\hline Debt-service in percent of fiscal revenue & - & $6 \%$ & $7 \%$ & $7 \%$ & $8 \%$ & $8 \%$ \\
\hline
\end{tabular}

Sources: Kenyan authorities; and staff estimates and projections.

1/ Based on external debt data available from the Kenyan authorities as of October 2004

2/ Excludes arrears.

3/ Refers to the present value of debt service calculated by using the currency-specific commercial interest reference rate (CIRR) as the discount rate.

4/ Three-year backward-looking average.

5/ Debt service due beforethe January 2004 Paris Club rescheduling, except where noted 
Table 10. Kenya: Actual and Projected Payments to the Fund, 2001-11 1/

(In millions of SDRs, unless otherwise indicated)

\begin{tabular}{|c|c|c|c|c|c|c|c|c|c|c|c|c|}
\hline & Overdue & 2001 & 2002 & 2003 & 2004 & 2005 & 2006 & 2007 & 2008 & 2009 & 2010 & 2011 \\
\hline Principal & $\ldots$ & 18.6 & 14.0 & 7.0 & 9.5 & 5.0 & 9.2 & 6.7 & 6.7 & 14.2 & 29.2 & 37.4 \\
\hline Outstanding Fund credit & $\ldots$ & 78.6 & 57.6 & 75.6 & 116.1 & 186.1 & 226.9 & 245.2 & 238.5 & 224.3 & 195.1 & 157.7 \\
\hline \multicolumn{13}{|l|}{ FCO in percent of: } \\
\hline Exports of goods and nonfactor services & $\ldots$ & 3.4 & 2.5 & 3.1 & 4.3 & 6.2 & 7.0 & 7.0 & 6.2 & 5.5 & 4.5 & 3.5 \\
\hline External public debt & $\ldots$ & 2.2 & 1.6 & 2.2 & 3.2 & 4.9 & 5.7 & 5.9 & 5.5 & 5.0 & 4.3 & 3.4 \\
\hline Gross official reserves & $\ldots$ & 9.6 & 7.5 & 7.5 & 12.0 & 17.0 & 18.8 & 19.1 & 17.5 & 15.8 & 13.2 & 10.1 \\
\hline GDP & $\ldots$ & 0.9 & 0.7 & 0.8 & 1.1 & 1.6 & 1.8 & 1.9 & 1.7 & 1.5 & 1.2 & 0.9 \\
\hline Quota & $\ldots$ & 29.0 & 21.2 & 27.9 & 42.8 & 68.6 & 83.6 & 90.3 & 87.9 & 82.6 & 71.9 & 58.1 \\
\hline \multicolumn{13}{|l|}{ Obligations (principal) in percent of: } \\
\hline Exports of goods and nonfactor services & $\ldots$ & 0.8 & 0.6 & 0.3 & 0.4 & 0.2 & 0.3 & 0.2 & 0.2 & 0.3 & 0.7 & 0.8 \\
\hline External public debt & $\ldots$ & 0.5 & 0.4 & 0.2 & 0.3 & 0.1 & 0.2 & 0.2 & 0.2 & 0.3 & 0.6 & 0.8 \\
\hline Gross official reserves & $\ldots$ & 2.3 & 1.8 & 0.7 & 1.0 & 0.5 & 0.8 & 0.5 & 0.5 & 1.0 & 2.0 & 2.4 \\
\hline GDP & $\ldots$ & 0.2 & 0.2 & 0.1 & 0.1 & 0.0 & 0.1 & 0.1 & 0.0 & 0.1 & 0.2 & 0.2 \\
\hline
\end{tabular}

Source: Fund staff estimates.

1/ The table assumes that the remaining amount of the PRGF arrangement of SDR 175 million agreed in November 2003 (which is SDR 150 million as of 10/31/04) will be disbursed,

along with an augmentation of SDR 50 milllion requested by the authorities, to be disbursed in two equal installements of SDR 25 million at the time of the completion of the first and second reviews, in addition to the SDR 25 million disbursments at the completion of each of those reviews expected under the original program.

Debt service to the Fund was estimated by the Kenya mission staff since FIN projections do not reflect the requested augmentation.

2/ Projections are based on current rates of charge, including burden-sharing charges where applicable, for purchases in the General Resources Account,

and on current interest rates for Poverty Reduction and Growth Facility (PRGF), and Trust Fund. The current SDR interest rate is assumed for net use of SDRs. 


\section{Letter of Intent}

Nairobi, December 6, 2004

Mr. Rodrigo de Rato

Managing Director

International Monetary Fund

Washington, D. C. 24031

Dear Mr. Rato:

1. The annexed Memorandum of Economic and Financial Policies (MEFP) describes our performance to date under the program supported by the Poverty Reduction and Growth Facility (PRGF) arrangement and outlines the policies we plan to implement during the remainder of 2004/05 (July/June). Our goal remains that of reducing poverty by creating an environment conducive to the promotion of strong economic and employment growth as outlined in the Investment Program for the Economic Recovery Strategy for Wealth and Employment Creation (ERS).

2. Considerable progress has been made under the program. A rebound in economic growth has begun. The increase in domestic debt has been arrested. However, major challenges remain, which we are addressing in the context of the program for 2004/05. In this regard, to mitigate the negative effect on the balance of payments resulting from the recent sharp rise in oil prices and drought, induced food imports, we would like to request an SDR 50 million augmentation of the access under the PRGF arrangement.

3. All the quantitative performance criteria for end-December 2003 were met, except for the continuous performance criteria on the contracting or guaranteeing of nonconcessional external long-term debt and on the accumulation of external payments arrears. With regard to the former, the nonconcessional external long-term debts that were contracted in contravention of the program understandings have been cancelled. The continuous quantitative performance criterion on the nonaccumulation of external arrears was breached after June 30, 2004 with regard to two categories of arrears. There was a late payment of the nonrescheduled share of Paris Club arrears existing at the time of the January 2004 rescheduling, which was required to be settled by July 1, 2004, but was not fully settled until November 2004. An accumulation of additional external arrears may result from a suspension of payments due to an audit of all central government contracts of commercial external debt that we are undertaking to enhance public expenditure management and good governance. While the audit is on-going, the debt-service due on these loans will be placed in an escrow account. We will use this account to resolve any arrears promptly and fully, once the underlying debt obligation is found by the audit to be legitimate.

4. All but one of the structural performance criteria related to the second disbursement have been completed, albeit two were completed with delays. The structural performance criterion on the completion of an audit on the financial position of the National Social 
Security Fund (NSSF) by end-December 2003 was completed in February 2004, and that on the submission to parliament of a Banking Act Amendment Bill by end-March 2004 was done in June 2004. Regarding the end-December 2003 structural performance criterion concerning the completion of an audit of the stock of pending bills and the adoption of measures to provide for their clearing, we completed the financial audit, but are awaiting the results of a legal audit of the pending bills before finalizing the clearance plan.

5. We request waivers for the nonobservance of the five performance criteria detailed above. We also request a rephasing of the remaining disbursements under the arrangement. This latter request reflects the delay in completing the first review under the program.

6. The MEFP spells out the quantitative performance criteria and benchmarks, as well as the structural performance criteria and benchmarks to be used in monitoring performance under the 2004/05 program. The end-September 2005 targets are indicative. These performance clauses will be firmed up once a more comprehensive macroeconomic framework, as well as the budget for 2005/06, is developed. The government is taking a number of prior actions to the issuance of the staff report on the first review of the PRGF arrangements.

7. The government believes that the policies set forth in the attached MEFP are adequate to achieve the objectives of its program, but it will take any further measures that may become appropriate for this purpose. Kenya will consult with the Fund on the adoption of these measures, and in advance of revisions to the policies contained in the MEFP, in accordance with the Fund's policies on such consultation. In particular, the government will consult with the Fund if it decides to proceed to implement any program that would result in a modification of the fiscal targets under the program. Kenya will continue to provide the Fund with all the information in a timely manner as required to monitor progress in implementing policies and achieving the objectives of the PRGF arrangement. We expect to complete the first progress report of our ERS by June 2005.

Sincerely yours, $/ \mathrm{s} /$

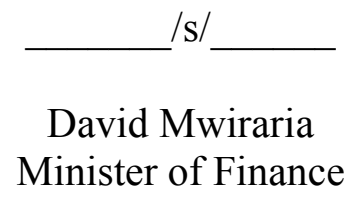

Attachment: - Memorandum of Economic and Financial Policies - Technical Memorandum of Understanding 


\section{KENYA}

\section{Memorandum of Economic and Financial Policies for 2004/05 Under the PRGF Arrangement}

\section{INTRODUCTION}

1. In 2003/04 (July/June), Kenya began to build the foundation for a new liberal democratic order, as well as for lasting economic prosperity. In the political arena, considerable effort was devoted to constitutional reform. The government is committed to completing the constitutional review as soon as possible because as a new constitution is essential to laying the legal basis for strengthening Kenya's governance structures and for sustained peace and stability - key ingredients for promoting strong economic growth and prosperity. On the economic front, an important beginning has been made to tackling Kenya's major macroeconomic vulnerabilities and addressing impediments to strong economic growth and poverty reduction. However, progress in implementing some key economic reforms has been impeded by the large amount of time devoted to constitutional reform, capacity constraints in key ministries, and the slow resumption of donor budgetary assistance.

\section{The government is committed to strong reforms, but the approach has been} modified to take account of lower budgetary support and capacity constraints. The government remains committed to implementing policies aimed at promoting strong economic growth and poverty reduction, as well as to fostering an environment conducive to private-sector-led growth and the expansion of employment opportunities. The government's approach is spelled out in the Investment Program for the Economic Recovery Strategy for Wealth and Employment Creation (ERS) - Kenya's PRSP, which was considered by the Executive Boards of the Fund and the World Bank (WB) in May 2004. The diagnosis of the main economic challenges facing Kenya, as well as the broad economic reform strategy, remains as outlined in the ERS and Memorandum of Economic and Financial Policies (MEFP) for 2003/04. This memorandum explains how the government reform agenda and the macroeconomic framework underpinning the PRGF-supported program have been modified to cope with the lower donor budgetary assistance now foreseen and the steps that will be taken to address capacity problems.

\section{Recent Economic Developments and Progress Under the Program}

3. Most of the performance criteria (PC) under the PRGF arrangement were met. All quantitative performance criteria for end-December 2003 were met; the continuous quantitative $\mathrm{PC}$ on the contracting or guaranteeing of nonconcessional external long-term debt was breached when two security-related clauses were concluded in December 2003, but these were cancelled shortly thereafter. The continuous PC on external payments arrears was breached after June 30, 2004, on to two classes of arrears. There was a late payment of the nonrescheduled share of Paris Club arrears existing at the time of the January 2004 rescheduling, which had to be settled by July 1, 2004, but was not fully paid until 
November 2004. Arrears also arose through an audit of all central government contracts of commercial external debt, which the government is conducting for governance and public expenditure management reasons; while the audit is proceeding these loans are not being serviced, resulting in relatively modest external arrears. The structural PC on the completion of an audit on the financial position of the National Social Security Fund (NSSF) by end-December 2003 was completed in February 2004, and that on the submission to Parliament of the Banking Act Amendment Bill by end-March 2004 was done in June 2004. Regarding the PC on the completion of an audit of the stock of pending bills and the adoption of measures to provide for their clearing, the financial audit was completed, but finalizing the clearance plan is awaiting the results of a legal audit of the pending bills that is expected to be completed by June, 2005.

4. The Letter of Intent (LOI) to which this MEFP is annexed requests waivers for the nonobservance of the five performance criteria detailed above and a rephasing of disbursements under the PRGF arrangement.

5. Economic developments in $\mathbf{2 0 0 3 / 0 4}$ were mixed. Real GDP growth at 2.1 percent was moderately above program expectations, despite the sharp increase in oil prices and the emergence of a drought late in the fiscal year. For the same reasons, overall consumer price inflation was well above projections. Excluding food and oil, inflation, at 4 percent at end-June 2004, was also higher than programmed, reflecting an easing of monetary policy. These outcomes together with only moderate donor inflows and higher oil prices, contributed to the depreciation of the nominal effective exchange rate of the shilling.

6. The easing of monetary policy in $2003 / 04$ to support economic recovery resulted in declining interest rates and rising inflation. Following the reduction in the legal reserve requirement from 10 percent to 6 percent in July 2003, the reserve money multiplier rose from 4.9 to 5.3, resulting in a 13 percent expansion of broad money in 2003/04 against the 7 percent projected under the program. The consequent rise in liquidity led to negative real yields on money market instruments. In response to the decline in interest rates, bank credit to the private sector grew substantially.

\section{Net domestic borrowing and the NPV of total central government debt (in} percent of GDP) were well within the program targets for 2003/04. This outcome reflected a number of developments:

- $\quad$ Stronger than budgeted revenue performance, which benefited, in part, from the aggressive collection of tax arrears and the implementation of new administrative measures;

- $\quad$ Tightened management of the recurrent budget, in the face of persistent wage pressures;

- Delays in the recapitalization of the National Bank of Kenya, which had been programmed to cost about Ksh 12 billion. 
8. Reflecting the slight decline in gross investment and a marked fall in the national savings ratio that was partly attributable to drought-related shifts in expenditure toward increased consumption, the external current account deficit (before grants) expanded relative to $2002 / 03$, but was lower than programmed in $2003 / 04$. The modest fall in gross investment was partly the result of lower public investment, because the pace of execution of the development budget was well below the budgeted level; problems in applying the transitional procurement system introduced early in the year; and lower than expected donor inflows that was partly the result of weaknesses in accounting for donor-funded development projects. A sharper than anticipated decline of private savings was partially offset by a significant increase in public savings. The buildup of foreign reserves was, in dollar terms, lower than the program target; however, it exceeded the target after an adjustment for the shortfall in external donor support.

9. Progress in implementing structural reforms has been mixed. There were some delays in implementing reforms in the public expenditure, financial sector, and parastatal areas. The lack of progress in moving toward a comprehensive medium-term expenditure framework, consistent with PRSP priorities, as well as in instituting a more robust expenditure management system, is largely attributable to organizational and capacity constraints at the Ministry of Finance. Financial sector and parastatal reforms suffered from capacity constraints and a lack of consensus on the objectives and modalities for conducting the reforms that has also delayed the enactment of the Privatization Bill. New wage-setting mechanisms for public employees have now been developed. Significant progress was made in building a robust governance architecture and in enforcing elements of the anticorruption regulations. Notable actions included the enactment of the Anti-Corruption and Economic Crimes and Public Officer Ethics Acts in May 2003, completion of asset declarations by all public officers in November 2003, major restructurings of the judiciary and the police over 2003/04, and the establishment of the Kenya Anti-Corruption Commission (KACC) in May 2004.

\section{Medium-Term Framework ANd Policies}

10. The medium-term macroeconomic framework underpinning the reform agenda has been modified to take account of the more difficult environment now foreseen for the medium term. In particular, (a) the terms of trade are now projected to deteriorate moderately instead of the modest gains envisaged earlier; (b) donor assistance would fall significantly short of earlier expectation; (c) capacity constraints in key areas are more severe than assumed under the program; and (d) some key reforms in the financial and parastatal sectors have taken longer to complete, partly because of the crowding out of the legislative agenda, in the recent past, by constitutional reform deliberations.

11. In response to these developments, the government has modified its reform strategy in several respects. The domestic revenue effort has been strengthened and expenditure restructuring and management reforms accelerated. In addition, the domestic borrowing targets have been relaxed somewhat. Although these changes will result in a moderate increase in domestic budgetary resources and in an improvement in the 
productivity of public spending, public investment will be scaled down over the medium term when compared to the original program assumptions. Partly for this reason, the realization of the economic and employment growth and poverty reduction objectives set out in the MEFP for 2003/04 would take longer to achieve.

12. Notwithstanding the adverse effects of the more difficult foreign environment, the external current account deficit would be lower than projected earlier as investment would be markedly lower than envisaged in the MEFP. On the other hand, the ratio of savings to GDP would be broadly as assumed earlier. The outlook for donor inflows would continue to dominate public investment activity, which is a key factor in promoting private sector activity. Partly for this reason and the slow response of private investment to the reforms, private investment would be significantly below program expectation. Reflecting the above developments, the net foreign reserve targets and the medium term would be somewhat less ambitious than foreseen in the original three-year program. The medium-term inflation objectives would remain as spelled out in last year's Economic Memorandum, although monetary policy will be tightened to correct for some overshooting in 2003/04.

\section{ECONOMic Policies For 2004/05}

\section{A. Macroeconomic Outlook}

13. A moderate pickup in economic activity is foreseen. Projected real GDP growth at 2.7 percent in 2004/05 would be lower than the 3.1 percent expected in the original program, reflecting in part the negative effects of a more difficult external environment - high oil prices and depressed prices for some traditional exports, as well as the impact of the drought on agricultural production. However, the tourism sector is projected to perform strongly as it recovers from the adverse effects of last year's travel ban. The drought and high oil prices are expected to contribute to a pickup in overall consumer price inflation to well above earlier projections.

\section{B. Fiscal Policy}

\section{The eventual lowering of the domestic debt ratio to GDP remains the key focus} of the program. The budget for 2004/05 that is before parliament targeted an overall deficit (before grants) of 7.2 percent of GDP and implied domestic borrowing of 4.7 percent of GDP (in the event that the envisaged budgetary support was not forthcoming). A revision to the budget announced on June 12, 2004 will be presented to parliament during the first quarter of 2005. Pending this revision, the new fiscal framework will be implemented through quarterly releases of expenditures. The revised budget assumes that domestic borrowing would amount to the equivalent of 2.5 percent of GDP. The fiscal framework envisages a marked decline in both the overall and primary deficits relative to ERS targets, wholly on account of the significant shortfall of donor budgetary support. For the same reason and the need to preserve some essential social and economic programs, domestic borrowing would exceed ERS expectations. Some crowding out of private activity is expected, as the public sector would absorb a sizable proportion of projected financial savings. Reflecting the projected domestic 
borrowing, domestic debt would remain broadly unchanged at 22 percent of GDP compared with the original program target of 24.7 percent of GDP. The domestic debt-ratio is expected to decline thereafter.

15. New administrative measures are projected to raise revenue collection. The coming into effect on January 1, 2005 of the East African Community (EAC) Customs Protocol involving a lowering of the top tariff rate from 35 percent to 25 percent and the reduction in tariff bands from 5 to 3 is projected to result in revenue losses of 0.3 percent of GDP. The program includes several measures aimed at raising compliance and expanding the tax base. These include:

- $\quad$ Creation of one-stop shops that bring together business licensing and tax payments operations;

- $\quad$ Full integration of the VAT and Income Tax Departments in a single function-based Domestic Tax Department (DTD);

- $\quad$ strengthening and rationalizing the Large Taxpayer Office (LTO);

- $\quad$ Introduction of computerized audits for LTO and DTD;

- Implementation, on a pilot basis, of simplified customs procedures; and

- Modernization of customs computer systems.

These measures, together with the ones implemented in 2003/04, are expected to strengthen revenue performance, significantly raise the efficiency of the tax system over the medium term, and lay the basis for a further reform of the tax system, beginning later this fiscal year. The medium-term goal is to broaden the tax base, as well as simplify and improve the neutrality and revenue-yielding capacity of the system.

\section{Government remains firmly committed to the reorientation of public} expenditures in favor of growth and poverty-related outlays. In line with this approach, government employment for critical social and economic sectors (including teachers, healthcare, and security personnel) will rise moderately but overall civil service positions would fall by about 2,000 through retrenchments and attrition. Several developments are expected to raise the inflexibility of the budget and thus limit the scope for a more aggressive reallocation of spending to priority areas. These include the projected rise in interest payments; the continuing sharp increase in pension payments that is partly attributable to the recent retrenchment of public servants; and the impact of persistent wage pressures on the wage bill; and the lower-than-expected external budget support. To stay on course with the expenditure restructuring program and move toward fiscal sustainability, the government has initiated several actions that should, over time, result in a significant reduction in noncore outlays. These include developing new wage-setting mechanisms for public employees, rationalizing diplomatic missions with a view to reducing the number of staff, and privatizing 
parastatals. In addition, the role of the state in the provision and funding of university education is under review. The budget includes a Ksh 2 billion provision for the payment of pending bills in 2004/05 following the completion of a legal audit in December 2004. However, if creditors contest the outcome of the audit, the process could take longer. The remaining bills will be paid off over a two-year period. The budget also includes a provision of Ksh 1 billion for the rehabilitation of stalled projects. Government's objective is to sell most of these projects and to rehabilitate the remaining ones in priority areas in the context of the regular development budget operations. As a result of the ongoing audit of all central government contracts of commercial external debt, some external payments arrears are accumulating. In managing the fiscal program, all the debt-service on these obligations will be paid on the due dates into an escrow account to facilitate prompt payment once the underlying obligations are found to be legitimate.

\section{A robust public expenditure management system is essential to putting the} economy on a sustainable, rapid, and poverty-reducing growth path and to increasing the transparency of public operations. Government has therefore initiated steps designed to build a sound expenditure management system, including:

- $\quad$ Completing the budget outlook paper for 2005/06 by November 2004 that would provide a link between the budget and ERS priorities;

- $\quad$ Expanding the comprehensiveness of the three-year medium-term expenditure framework (MTEF), as well as the coverage of the programs included in the protected social programs;

- $\quad$ Strengthening the links between the MTEF and the annual budget system;

- Improving budget transparency by adopting the classification system proposed in the Government Financial Statistics Manual for all budget votes;

- $\quad$ Broadening the consultative mechanisms used in the preparation of sectoral programs and medium-term budgets;

- $\quad$ Creating over time, an Integrated Financial Management System, while strengthening the expenditure commitment control system (ECCS) in the interim. Full application of the ECCS will help to prevent a build up of new arrears; and

- $\quad$ Strengthening procurement systems and accounting capacity and practices in line ministries, as well as procedures. The government is doing its utmost to facilitate the enactment of the Procurement Bill by March 2005.

18. Public service reforms have been stepped up. This will help to re-establish control over the wage bill, address capacity constraints in key ministries, and promote the creation of a more efficient public service. To this end, the government has introduced new wage-setting guidelines for public employees, consistent with reducing, over the medium term, the wage 
bill as a proportion of revenue over the medium term. The terms and conditions of service for top management in the government and state bodies will also be streamlined. Finally, new regulations emphasizing merit as a key determinant for promotion have been established. Capacity building is being emphasized at all levels of government, particularly in ministries essential to the realization of the ERS. In this connection, several steps have been taken to strengthen capacity and streamline the Ministry of Finance, including consolidation of the planning and budget functions within the Ministry, the setting up of a robust debt management unit, and enhancing capacity and mechanisms for managing and monitoring macroeconomic and structural reforms. With the assistance of private consultants, the Ministries of Education, Health, and Agriculture are also being restructured.

\section{Monetary Policy}

19. The monetary program for $\mathbf{2 0 0 4 / 0 5}$ is designed to reduce underlying inflation to 3.5 percent. The inflation objective would be sought largely through reserve money targeting, with broad money (M3X) as the intermediate target and open market (repurchase and reverse) operations as the main instrument. Assuming that the money multiplier increases moderately in response to the expected pickup in interest rates, the program is consistent with targeting a growth rate of reserve money of 3.8 percent. To implement the monetary program, the CBK will sterilize, on a timely basis, any excess liquidity, thereby leaving interest rates to be determined by market forces in line with the monetary program. With Fund technical assistance, the auction system for government bonds will be improved. The government will continue to implement a flexible exchange rate system, which has served the economy well in absorbing fluctuations in external conditions. CBK's intervention in the foreign exchange market will be limited to meeting the net foreign assets target under the program and to smoothing disruptive short-term fluctuations.

\section{Structural Reforms}

20. The measures under the program will reinforce government efforts to promote good governance. The following are the main elements of the strategy:

- Continued strengthening of the governance architecture and enforcement of key anticorruption laws and regulations. This includes full enforcement of the provisions of the Anti-Corruption and Economic Crimes and Public Officer Ethics Acts 2003, as well as implementation of the United Nations Convention against Corruption and Ratification of the African Union Convention on Corruption.

- $\quad$ Enhancing the effectiveness of key anticorruption investigative agencies. These include the Kenya Anti-Corruption Commission, the government's principal investigative body whose capacity has been greatly improved since the swearing in of senior officials in September 2004. An additional step entails the hiring of key personnel, targeted for completion by July 2005. 
- Continuing efforts to build adequate prosecution capacity for handling the expected expansion in corruption-related case-loads. The primary focus is on the Department of Public Prosecution, the government's principal prosecuting authority, which has recently established separate units to address corruption, serious crime, fraud, and asset forfeiture. Completion of the capacity-building program is targeted for early 2005 .

- Developing systems for annual declaration and verification of wealth, assets, and liabilities of ministers and other senior public officials as provided for under the Public Officers Ethics Act 2003. The strategy includes computerization of wealth declaration forms which has already been initiated; the submission to Parliament by April 2005 of an amendment to the Act to provide for the annual verification of asset declarations by the KACC; and follow-up on the commitment by the Cabinet Committee against Corruption in November 2004 to the voluntary publication of assets.

- Developing a robust program of asset recovery and restitution. The Kenyan restitution strategy is designed to follow the standard three distinct phases, namely, international freezing of assets; restitution and vesting in the aggrieved country; and domestic recovery of returned assets, if nonmonetary. A restitution advisory group comprising the Department of Governance and Ethics, the Kenya Anti-Corruption Commission, and the Department of Public Prosecutions has been established.

- Deepening transparency in the conduct of public operations. Elements of the program include strengthening the public sector budget and financial management system through timely production of accounts by all public institutions, auditing and presentation to Parliament of the accounts, and production of comprehensive annual and medium-term expenditure frameworks, consistent with ERS priorities.

- Improving the procurement system, including those for security-related operations. Key aspects of the program are enactment by Parliament of the Public Procurement and Disposal Bill 2004, which would also provide for the establishment of the Public Procurement Oversight Authority (PPOA), with autonomy on matters of Public Procurement; formulation of new Public Procurement and Disposal regulations to implement the Act; development of a public procurement Directorate/Oversight Authority Strategic Plan 2005-10; preparation of the Training and Capacity-Building Program for procurement personnel in the public sector and an action plan for the proposed public procurement reforms activities, in consultation with stakeholders; and a deepening of the transparency of the procurement process by expanding the participation of stakeholders in the oversight of the public procurement process, and establishing an appeals body to deal with complaints; periodic media publication of the awarding of contracts in the media; and the development of IT capacity to facilitate dissemination of public procurement information to the public. 
- $\quad$ Addressing the outcomes of recent initiatives to address past economic crimes. Major areas of focus are the Goldenberg Commission of Inquiry, which has begun to throw light on the single most debilitating series of corrupt transactions in Kenyan history, and the Presidential Commission on Illegal and Irregular Allocations of Public Land.

- $\quad$ Further upgrading key public agencies. The strategy includes the implementation of the recommendations of the task force on reform of the police beginning December 2004, addressing issues of retraining, strengthening oversight mechanisms, and corruption; corruption prevention in the civil service initiated under the auspices of the Public Service Integrity Programme (PSIP) of the Directorate of Personnel Management of the Office of the President in conjunction with the KACC and strengthening the Governance Justice Law and Order Sector Reform Program, a sectorwide development strategy based on recognition of the need for contemporaneous reforms within the sector as a whole.

- Enhancing public interaction with the government on governance. This will involve a Public Complaints Unit (PCU) to serve as a central referral and monitoring and evaluation unit under the aegis of the Department of Governance and Ethics, in conjunction with the Ministry of Justice and Constitutional Affairs.

21. The program will set the stage for an acceleration of parastatal reforms once the Privatization Bill is enacted. The government considers parastatal restructuring and privatization as an important element of its ERS. Pending parliamentary consideration and ratification of the bill — now foreseen by April 2005- that will institutionalize a more transparent mechanism for privatizing public enterprises, the government will initiate by December 2004 a comprehensive review of the financial position of key parastatals. In addition to identifying the contingent liabilities of the government, the information produced by the review will be essential in developing privatization options. Moreover, following the recent setting up of a Bank Restructuring and Privatization Unit in the Ministry of Finance, the restructuring of the National Bank of Kenya has begun. As part of this process, at the next Annual General Meeting of the NBK board around, April 2005, all state nominated Board members of the bank will be replaced by independent professional directors; and pending completion of the restructuring process. NBK will desist from disposing assets, and raising its liabilities with CBK from the end-June 2004 position. In addition, its outstanding loans and advances will not exceed the end-June 2004 level. Moreover, the government will prepare by March 2005 a time-bound plan for divesting public ownership and control of other public banks.

22. The government is implementing measures designed to strengthen the financial system. Amendments to the $\mathrm{CBK}$ and Banking Acts, which are before parliament, provide for the transfer of virtually all banking sector regulatory and supervisory authority from the Ministry of Finance to the Central Bank; the removal of a section of the Banking Act requiring prior approval of the Minister of Finance when setting banks' fees and charges; and changes in the definition of a significant shareholder, as well as in the fit-and-proper 
criteria for managers and significant shareholders. New regulations tightening loan provisioning and classification will be introduced in January 2005. In addition, a review of the management and financial position of the NSSF will be completed by March 2005 that will set the stage for the restructuring of the Fund.

23. Several actions are under consideration to enhance Kenya's competitiveness position. The current practice of raising the minimum wage on a yearly basis will be discontinued. Henceforth, the minimum wage will be reviewed once every two-year period, on the basis of wage trends in the informal sector and formal sector productivity gains for unskilled labor. In addition, new guidelines for wage arbitration by the Industrial Court will be issued by March 2005 that will emphasize productivity change as the key criterion for wage adjustments.

\section{The government will continue to emphasize trade reform and regional} integration. In the context of the EAC, the external tariff and number of tariff bands will be reduced, which will result in a fall in the average tariff rate from 16.2 percent to 10.9 percent. As a result, and after taking account of the absence of non-tariff-barriers, Kenya's rating under the IMF's trade restrictiveness index is expected to fall significantly, resulting in a more open trade regime. This will also help to foster a more competitive environment. Furthermore, the government will, by December 2004, complete a trade policy strategy that will spell out the reforms needed to enhance efficiency and international competitiveness, including additional trade reforms in the context of the East African Community. Many of the measures covered above are also part of a broad package of reforms that the government is taking to improve the investment climate in Kenya. Capacity building in the government will result in better quality public services. Improved governance and better fiscal management will translate into improved private sector confidence in the Kenyan economy. Many priority projects under the ERS, such as the upgrading of the road network, the concessioning of railway operations, and streamlining of port operations, will feed directly into an improved business environment by lowering the costs of production.

\section{E. Poverty and Social Impact Analysis}

25. Poverty and social impact analysis (PSIA) of key policy reforms will be conducted. Terms of reference for a PSIA of the EAC tariff reform and a possible further lowering of the tariff rates within the context of the $\mathrm{EAC}$, is under consideration. The study will assess the likely impact of the trade reforms on the cost of living of the poor, the main economic sectors, employment, and the regions likely to suffer the most. This will help to fine tune the reforms and the development of appropriate mitigating measures.

\section{F. External Financing}

26. While lower than projected in the November 2003 MFEP because of the scaling down in projected investment, the external financing requirements for 2004/05 of US\$ 1,245 million incorporates the effects of higher import payments stemming from 
the increase in oil prices and drought-related food imports. The economic reform agenda and the government's commitments under the program that is supported by the PRGF arrangement are expected to assist in mobilizing donor financial support. The World Bank has indicated in its May 2004 Country Assistance Strategy for Kenya a base-case commitment of US $\$ 140$ million of budgetary support under the Programmatic Structural Adjustment Credit (PSAC) and Financial Sector Adjustment Credit (FSAC) facilities, with a disbursement of US\$75 million expected in late FY2004/05, and of US\$65 million in early FY2005/06. The AfDB has indicated that it plans to disburse US\$21 million of program support in 2004/05, and the European Union is projected to disburse US\$60 million of program support in early 2005. Disbursement of these resources, as well as the envisaged project support and private financing in FY2004/05, under the PRGF arrangement and the debt relief of about US\$130 million from Paris Club creditors, will cover the estimated financing need of about US\$ 1.2 billion in 2004/05.

\section{G. Safeguards and Statistics Issues}

27. The government remains committed to protecting the financial soundness of the CBK, and to adhering to the principles of good governance and best practices, including those encapsulated in the IMF's safeguards policy. A safeguards assessment of the CBK conducted in September 2003, found that the Bank had made notable progress in strengthening its safeguards since the December 2000 on-site assessment. The bank is committed to implementing measures to address the remaining vulnerabilities, and to further strengthening the CBK's operations.

28. On statistical issues, the government has released a revised set of national accounts data. The government is also taking steps to improve the monitoring of fiscal data, in particular, the preparation, on a regular basis, of summary statistics on domestic arrears and pending bills. Moreover, the government intends to develop a framework for systematically monitoring productivity changes in the economy, and to produce, on a regular basis, trade data. Kenya participates in the General Data Dissemination System project for Anglophone Africa. A statistics ROSC will be conducted in early 2005.

\section{H. Program Monitoring for 2004/05}

29. Technical memorandum of understanding (TMU). The program will be monitored using the definitions, data sources, and frequency of monitoring set out in the accompanying TMU. The government will make available to Fund staff all data appropriately reconciled and on a timely basis, as specified in the TMU.

30. Prior actions. The government is taking a number of actions prior to the issuance of the staff report on the first review of the PRGF arrangement. The prior actions are described in this memorandum (Table 3).

31. Performance criteria and benchmarks. Table 4 shows the quantitative performance criteria and benchmarks to be used in monitoring performance in 2004/05. Structural performance criteria and benchmarks, with corresponding dates, are identified in Table 3 . 
32. Program review. The second review under the PRGF arrangement will be completed no later than end-June 2005. This review will focus on the restructuring of expenditure in favor of social and economic spending; actions to enhance competitiveness; progress in rationalizing the financial system; progress in improving the auction system for government debt-instruments taking into account the recommendations of the November 2004 MFD technical assistance mission; and the fiscal implications of restructuring the National Social Security Fund. The third review under the PRGF arrangement is expected to be completed no later than end-December 2005. 
Table 1. Kenya: Structural Performance Criteria and Benchmarks Under the PRGF Arrangement for 2003/04

\begin{tabular}{|c|c|c|}
\hline & Implementation Date & Status \\
\hline \multicolumn{3}{|l|}{ Prior Actions } \\
\hline $\begin{array}{l}\text { Presentation to parliament of a budget for 2003/04 } \\
\text { consistent with program understandings. }\end{array}$ & End June 2003 & Observed \\
\hline $\begin{array}{l}\text { Government to decide on transfer of financial } \\
\text { sector regulatory functions from the Ministry of } \\
\text { Finance to the Central Bank of Kenya (CBK). }\end{array}$ & End June 2003 & Observed \\
\hline $\begin{array}{l}\text { Reaching an understanding with staff on a time } \\
\text { bound plan for restructuring the National Bank of } \\
\text { Kenya (NBK). }\end{array}$ & End June 2003 & $\begin{array}{l}\text { Observed. Subsequently, differences arose } \\
\text { between Fund and Bank programs. }\end{array}$ \\
\hline \multicolumn{3}{|l|}{ Structural Performance Criteria } \\
\hline $\begin{array}{l}\text { Finalize an audit of the stock of pending bills and } \\
\text { adopt measures that provide for the clearing of the } \\
\text { stock of pending bills over a three-year period. }\end{array}$ & End December 2003 & $\begin{array}{l}\text { Not observed. Financial audit completed but } \\
\text { clearance plan to await conclusion of legal } \\
\text { audit. }\end{array}$ \\
\hline $\begin{array}{l}\text { Completion of a report of the Committee of } \\
\text { Officials on the new mechanism for determining } \\
\text { the salaries of public officials to ensure the } \\
\text { reduction of the wage bill in total expenditure. }\end{array}$ & End December 2003 & Observed \\
\hline $\begin{array}{l}\text { Completion of an audit on the financial position of } \\
\text { National Social Security Fund (NSSF). }\end{array}$ & End December 2003 & $\begin{array}{l}\text { Not observed. Audit was completed in March } \\
2004\end{array}$ \\
\hline $\begin{array}{l}\text { Submission to Parliament of a Banking Act } \\
\text { Amendment Bill providing for transfer of all } \\
\text { financial sector regulatory functions from the } \\
\text { Ministry of Finance to the CBK. } \\
\text { No imposition of controls by the government or the } \\
\text { CBK on the determination by commercial bank of } \\
\text { bank fees, charges, or interest rates. }\end{array}$ & End March 2004 & $\begin{array}{l}\text { Not observed. Draft legislation was held up in } \\
\text { Attorney General's office on account of } \\
\text { limited drafting capacity. Presented to } \\
\text { Parliament in June } 2004 \text {. } \\
\text { Observed }\end{array}$ \\
\hline \multicolumn{3}{|l|}{ Structural Benchmarks } \\
\hline $\begin{array}{l}\text { Presentation of } 2002 / 03 \text { final budgetary accounts to } \\
\text { the Auditor General. }\end{array}$ & End March 2004 & Observed \\
\hline $\begin{array}{l}\text { Reaching an understanding with staff on new wage } \\
\text { setting mechanisms for public employees designed } \\
\text { to reduce the share of the wage bill in total } \\
\text { expenditure. }\end{array}$ & End March 2004 & $\begin{array}{l}\text { Not observed. New wage setting mechanism } \\
\text { agreed and incorporated in program for } \\
2004 / 05 \text {. }\end{array}$ \\
\hline $\begin{array}{l}\text { Presentation to parliament of a bill to establish a } \\
\text { framework for the transparent privatization and } \\
\text { sale of public assets. }\end{array}$ & $\begin{array}{l}\text { September/December } \\
2003\end{array}$ & $\begin{array}{l}\text { Not observed. Privatization bill presented to } \\
\text { Parliament in January } 2004 .\end{array}$ \\
\hline $\begin{array}{l}\text { Development of an action plan and timetable for } \\
\text { introducing a Commitment Control System (CCS) } \\
\text { to minimize deviations of expenditure outcomes } \\
\text { from targets and the buildup of arrears. }\end{array}$ & End December 2003 & $\begin{array}{l}\text { Partially observed. Most of the elements of a } \\
\text { CCS have been implemented. A technical } \\
\text { evaluation of the current expenditure control } \\
\text { system to identify required actions has not yet } \\
\text { been undertaken. }\end{array}$ \\
\hline $\begin{array}{l}\text { Establishment of a clear timetable for completion } \\
\text { of initial asset declarations by all senior public } \\
\text { officials. }\end{array}$ & End December 2003 & $\begin{array}{l}\text { Observed. Initial declarations completed in } \\
\text { November } 2004 .\end{array}$ \\
\hline $\begin{array}{l}\text { Establishment of the Kenya Anticorruption } \\
\text { Commission (KACC), with a credible } \\
\text { management. }\end{array}$ & End December 2003 & $\begin{array}{l}\text { Not observed. KACC became operational in } \\
\text { September } 2004 \text {. }\end{array}$ \\
\hline $\begin{array}{l}\text { Reaching of understandings on the fiscal } \\
\text { implications of restructuring the NSSF. }\end{array}$ & End March 2004 & $\begin{array}{l}\text { Not observed. NSSF restructuring to be } \\
\text { undertaken in the context of the second } \\
\text { World Bank FSAC beginning 2005/06. }\end{array}$ \\
\hline
\end{tabular}


Table 4. Kenya: Proposed Quantitative Performance Criteria and Benchmarks Under the Program for 2004/05 1/ 2/

(In millions of Kenya shillings)

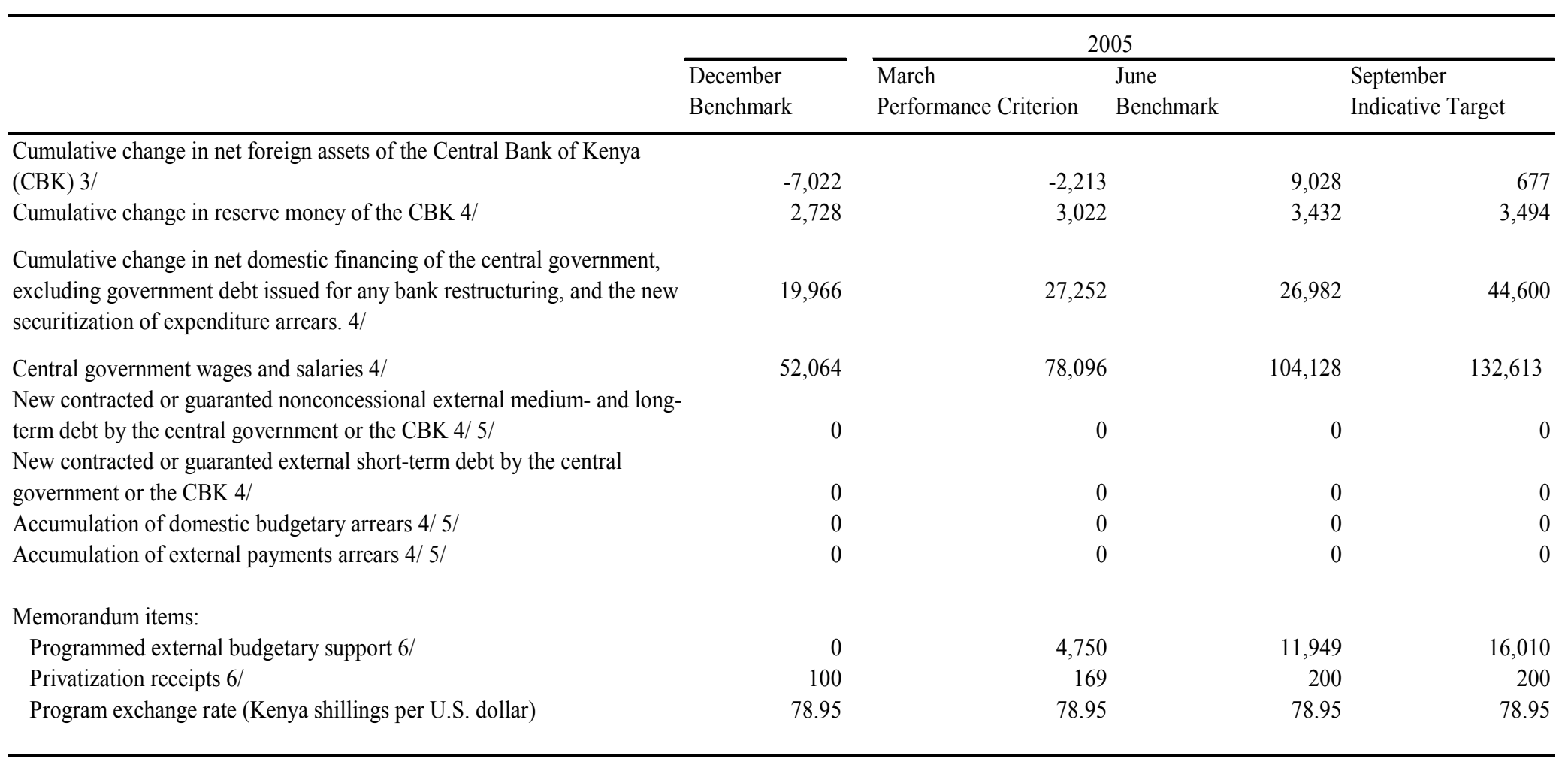

1/ The fiscal year begins on July 1, 2004.

2/ The performance criteria and benchmark under the program, and their adjusters, are defined in the technical memorandum of understanding

(TMU). All test dates for the performance criteria and benchmarks are on an end-of-period basis.

3/ Floor.

4/ Ceiling.

5/ Continuous performance criterion.

6/ Cumulative. 
Table 5: Kenya. Disbursement Schedule Under the Three-Year PRGF Arrangement

\begin{tabular}{|c|c|c|c|}
\hline Disbursement & $\begin{array}{c}\text { Amount } \\
\text { (In millions of SDRs) }\end{array}$ & Date & $\begin{array}{l}\text { Contingent on } \\
\text { Board Approval }\end{array}$ \\
\hline $1 \mathrm{st}$ & 25 & November 2003 & November 2003 \\
\hline 2nd & 50 & December 2004 & $\begin{array}{l}\text { end-December } 2003 \text { performance } \\
\text { criteria (PC) }\end{array}$ \\
\hline $3 \mathrm{rd}$ & 50 & July 1, 2005 & $\begin{array}{l}\text { end-March } 2005 \text { PC, and completion } \\
\text { of the second review by end- } \\
\text { June } 2005\end{array}$ \\
\hline 4 th & 25 & January 1, 2006 & $\begin{array}{l}\text { end-September } 2005 \text { PC, and } \\
\text { completion of the third review by } \\
\text { end-December } 2005\end{array}$ \\
\hline 5 th & 25 & July 1, 2006 & $\begin{array}{l}\text { end-March } 2006 \text { PC, and completion } \\
\text { of the fourth review by end- } \\
\text { June } 2006\end{array}$ \\
\hline $6^{\text {th1 }}$ & 25 & January 1, 2007 & $\begin{array}{l}\text { end-September } 2006 \mathrm{PC} \text {, and } \\
\text { completion of the fifth review by } \\
\text { end-December } 2006\end{array}$ \\
\hline $7 \mathrm{th}^{1}$ & 25 & July 1, 2007 & $\begin{array}{l}\text { end-March } 2007 \text { PC, and completion } \\
\text { of the sixth review by end-June } 2007\end{array}$ \\
\hline Total & 225 & & \\
\hline
\end{tabular}

${ }^{1}$ While these disbursements at present fall outside the period of the arrangement, it is expected that the authorities will in the future request an extension of the arrangement. 


\section{KENYA}

\section{Technical Memorandum of Understanding}

\section{INTRODUCTION}

1. This memorandum sets out the understandings between the Kenyan authorities and the International Monetary Fund (IMF) regarding the definitions of the quantitative and structural performance criteria and benchmarks for the second annual program (for 2004/05, July to June) under the three-year arrangement supported by the Poverty Reduction and Growth Facility (PRGF), as well as the related reporting requirements.

\section{Quantitative Performance Criteria And Benchmarks}

\section{A. Net Foreign Assets (NFA) of the Central Bank of Kenya (CBK)}

2. Definition. The NFA of the CBK are defined as the average over the calendar month of the test dates of the difference between the Central Bank of Kenya (CBK) holdings of gross official reserves (excluding crown agents and reserve tranche, pledged, swapped, or any encumbered reserves assets, including but not limited to reserve assets used as collateral or guarantees for third-party external liabilities) and its foreign liabilities (including IMF loans and other short- and long-term liabilities) of the CBK. Exchange rates prevailing on September 28, 2001 will be used to convert the NFA into Kenya shillings at the end of each test period. Table 4 in Annex I of the Letter of Intent, dated December 6, 2004, shows the floors on NFA, specified in Kenya shillings, for December 31, 2004, March 31 and June 30, 2005, as well as indicative targets for September 30, 2005.

3. Adjustment clauses. The NFA floors will be adjusted upward (or downward) by the sum of (i) excess (shortfall) in non-project-related external budgetary support relative to the programmed amount; and (ii) excess (shortfall) in privatization proceeds from external sources. Excess or shortfall proceeds are defined as the difference between actual and programmed proceeds. The downward adjustment is capped at the equivalent of US\$280 million, converted to Kenya shillings at the program exchange rate prevailing on September 28, 2001. All targets are cumulative from July 1, 2004 onward.

4. Reporting requirements. Data on gross international reserves, encumbered reserves, and foreign liabilities of the CBK will be transmitted to the Fund on a weekly basis within five working days of the end of each week. Detailed data on external project-related and nonproject-related budgetary support (by donor/creditor and by currency of denomination) will be transmitted on a monthly basis within five working days of the end of each week.

\section{B. Reserve Money (RM) of the Central Bank of Kenya}

5. Definition. RM is defined as the average over the calendar month of the test dates of reserve money, defined as total currency outside banks and bank reserves including till cash 
with the CBK. Table 4 in Annex I of the Letter of Intent shows the ceilings on RM for September 30, and December 31, 2004, and for March 31, 2005 and June 30, 2005, as well as indicative targets for September 2005. The authorities will consult with the Managing Director of the Fund on any changes in the ratio of reserve requirements.

6. Reporting requirement. The daily balance sheets of the $\mathrm{CBK}$ will be transmitted on a weekly basis within five working days of the end of each week.

\section{Net Domestic Financing (NDF) of the Central Government}

7. Definition. NDF includes financing by the banking system (the CBK and commercial banks), non-bank financial institutions, and the nonbank public of the budget of the central government of Kenya; where the central government is defined as in the Appropriation Accounts and in the parliament approved budget estimates for 2004/05. ${ }^{11}$ NDF consists of treasury bills, government stocks and bonds, promissory notes and other domestic debt instruments issued by the government, and loans and advances net of government deposits with the CBK and the banks. For the purposes of the program, NDF excludes privatization proceeds not included in the budget, and government debt issued for any bank restructuring. NDF is calculated as the cumulative change from July 1, 2004 onward in the sum of (i) loans and advances to the government by the CBK, including any interest arrears, minus all government deposits with the $\mathrm{CBK}$, from the balance sheet of the $\mathrm{CBK}$; (ii) loans and advances to the government by the commercial banks, including any interest arrears, minus all government deposits held with the banks, from the balance sheet of the commercial banks; and (iii) the changes in the outstanding stock of treasury bills, government stocks and bonds, promissory notes and other domestic debt instruments issued by the government, including any interest arrears. Table 4 in Annex I of the letter of intent shows the ceilings on NDF for September 30, and December 31, 2004, March 31 and June 30, 2005, as well as indicative targets for September 2005.

8. Adjustment clauses. The ceiling on NDF will be adjusted upward (downward) by the shortfall (excess) in non-project-related external budgetary support; The value of the cumulative excess or shortfall in external non-project-related budgetary support will be converted into Kenya shillings at the exchange rate prevailing at the time of disbursement. The externally caused part of any upward adjustment in NDF is capped at the equivalent of US\$ 280 million less any shortfall in external privatization receipt, converted to Kenya shillings at the exchange rate prevailing at the time of disbursement.

9. Reporting requirements. Data on NDF of the central government will be submitted to Fund staff on a monthly basis within four weeks of the end of each month.

${ }^{11}$ The 2004/05 Estimates of Revenues, 2004/05 Estimates of Recurrent Expenditures, and 2004/05 Estimates of Development Expenditures. 


\section{Central Government Wages and Salaries}

10. Definition. Central government wages and salaries consist of all compensation of central government employees (excluding social contributions paid by employer). Table 4 in Annex I of the Letter of Intent shows the ceilings on central government wages and salary payments for September 30 and December 31, 2004, March 31 and June 30, 2005, as well as indicative targets for September 30, 2005. For the purpose of this definition, the central government of Kenya is defined as in the Appropriation Accounts and in the parliament approved budget estimates for 2004/05.

11. Reporting requirement. Data on central government wages and salaries will be provided on a monthly basis within three weeks of the end of each month.

\section{E. Contracting or Guaranteeing of New Nonconcessional External Medium- and Long-Term Borrowing Public External Debt}

12. Definition. This performance criterion not only applies to debt as defined in point No. 9 of the Guidelines on Performance Criteria with Respect to Foreign Debt (see Decision of the Executive Directors of the IMF No. 12274-00/85, August 24, 2000) but also to commitments contracted or guaranteed for which value has not been received. It applies to debt contracted or guaranteed with original maturities of more than one year by the CBK or the general government of Kenya (as defined by the 2001 IMF Government Finance Statistics Manual). It excludes financial obligations to the Fund, domestically issued Kenya Shilling denominated treasury bonds with maturity greater than a year held by non-residents, and debt subject to debt rescheduling and debt reorganization. The ceiling for new nonconcessional external debt will be zero on a continuous basis throughout 2004/05.

13. For program purposes, a debt is concessional if it includes a grant element of at least 35 percent, calculated using currency-specific commercial interest reference rates (CIRRs) published by the OECD. For debt with maturity of at least 15 years, the ten-year average CIRR will be used, and for debt with maturity of less than 15 years, the six-month average CIRR will be used.

14. Reporting requirement. Data on all new debt and guarantees by the CBK and the general government (including terms of loans and creditors) will be provided on a monthly basis within three weeks of the end of each month.

\section{F. New External Nonconcessional Short-Term Debt Contracted or Guaranteed by the General Government or the Central Bank of Kenya}

15. Definition. This performance criterion not only applies to debt as defined in point No. 9 of the Guidelines on Performance Criteria with Respect to Foreign debt (see Decision No. 12274-00/85, August 24, 2000) but also to commitments contracted or guaranteed for which value has not been received. It applies to nonconcessional (see paragraph 14 above) debt with original maturities of up to and including one year contracted or guaranteed by the CBK or the general government (as defined by the 2001 IMF Government Finance Statistics 
Manual) of Kenya. It excludes financial obligations to the Fund, normal trade-related credits, and domestically issued Kenya Shilling denominated treasury bills with maturity of less than a year held by non-residents. The ceiling for new external nonconcessional short-term debt will be zero on a continuous basis throughout 2004/05.

16. Reporting requirement. Data on all new debt and guarantees by the CBK and the general government (including terms of loans and creditors) will be provided on a monthly basis within three weeks of the end of each month.

\section{G. Domestic Budgetary Arrears}

17. Definition. Domestic budgetary arrears are defined as the sum of all due and unpaid obligations of the central government of Kenya, where the central government is defined as in the Appropriation Accounts and in the Parliament approved budget estimates for 2004/05, including, but not limited to, payment obligations from procurement contracts for goods and services and other contracts providing for payment in domestic currency, as well as statutory obligations for payment (e.g., of domestic debt, domestic interest, civil service wages, and other entitlements). Changes in domestic budgetary arrears are defined as the stock of outstanding domestic arrears on the test date minus the stock of outstanding domestic arrears as of June 30, 2004. Under the program, the nonaccumulation of new domestic budgetary arrears is a continuous performance criteria.

18. Reporting requirement. Detailed data on repayment and new accumulation of domestic budgetary arrears and the remaining previous-year stock of domestic budgetary arrears will be transmitted on a monthly basis within four weeks of the end of each month.

\section{H. External Payments Arrears (On Nonreschedulable Debt)}

19. Definition. External payments arrears are defined as the non-payment of external obligations of the CBK or the general government of Kenya (general government as defined by the 2001 IMF Government Finance Statistics Manual) both with regard to principal and interest that are valid and due, but shall exclude arrears on obligations that are subject to rescheduling or restructuring. Under the program, the nonaccumulation of new external payments arrears is a continuous performance criteria.

20. Reporting requirement. Detailed data on the repayment and new accumulation of external payments arrears by the $\mathrm{CBK}$ and the general government and the stock of arrears at the beginning and end of each month will be transmitted on a monthly basis within four weeks of the end of each month.

\section{OTHER Reporting REQUiREMEnTS FOR PROGRAM MONITORING}

\section{Public Finance}

21. Monthly data on domestic financing (bank and nonbank) of the budget (including treasury bills and government bonds held by the nonbank public), domestic and external 
privatization proceeds, government debt instruments issued for bank restructuring, and new securitization of expenditure arrears will be transmitted on a monthly basis within three weeks of the end of each month.

22. Data on the implementation of the development budget, with detailed information on the sources of financing, will be transmitted on a quarterly basis within four weeks of the end of each quarter.

23. Public sector external and domestic scheduled debt service and payments will be transmitted on a monthly basis within three weeks of the end of each month.

\section{J. Monetary Sector}

24. The following data will be transmitted on a daily/weekly basis within one/five working day of the end each day/week:

- $\quad$ Treasury-bill rates (weekly); and

- Interbank interest rate (daily).

25. The following data will be transmitted on a monthly basis or as specified below within four weeks of the end of the month:

- The consolidated balance sheets of deposit money banks and the individual bank balance sheet, as needed;

- $\quad$ The monetary survey;

- $\quad$ Lending and deposit rates;

- Standard bank supervision indicators for banks and nonbank financial institutions, respectively, and for individual institutions as needed; and

- $\quad$ Net foreign assets of commercial banks and nonbank financial institutions, broken down by instruments.

\section{K. External Sector}

26. The following daily buying, selling, and average exchange rates will be transmitted on a weekly basis within five working days of the end of each week: (i) exchange rates used in interbank transactions among the commercial banks; (ii) the average of (i) (official exchange rate, quoted daily based on the transactions of the previous day); and (iii) foreign exchange purchases and sales by the CBK on interbank market and other inflows and outflows of foreign exchange to/from the CBK, separately. 
27. Export and import data, including volumes and prices, will be transmitted on a quarterly basis within eight weeks of the end of each quarter.

28. Other balance of payments data, including the data on services, private transfers, official transfers, and capital account transactions, will be transmitted on a monthly basis within four weeks of the end of each month.

\section{Real Sector}

29. Monthly disaggregated consumer price indices for Nairobi (Central Bureau of Statistics) will be transmitted on a monthly basis within four weeks of the end of each month.

30. Any revisions to the national accounts data will be transmitted within three weeks of the date of revision.

31. Data on real sector leading indicators will be transmitted within three weeks of the end of each month.

32. Data on employment (formal and informal) and wages (including minimum wages and collective wage agreements) will be transmitted within four weeks of the end of each month.

\section{Governance and Other Structural Reforms}

Documentation of all measures undertaken by the government will be transmitted to the IMF's African Department within five working days after the day of implementation. 


\section{Kenya: Relations with the Fund}

(As of September 30, 2004)

I. Membership Status: Joined February 3, 1964; Article VIII.

II. General Resources Account:

Quota

Fund holdings of currency

Reserve position in Fund

III. SDR Department:

Net cumulative allocation

Holdings

IV. Outstanding Purchases and Loans:

Poverty Reduction and Growth Facility (PRGF) arrangements
SDR million

271.40

258.71

12.70

SDR million

36.99

0.19

SDR million

70.83
Percent of quota

100.00

95.32

4.68

Percent of allocation

100.00

0.52

Percent of quota

26.10

V. Financial Arrangements:

$\begin{array}{lcccc} & \text { Approval } & \text { Expiration } & \text { Amount Approved } & \text { Amount Drawn } \\ \text { Type } & \text { Date } & \text { Date } & \underline{\text { (SDR million) }} & \text { (SDR million) } \\ \text { PRGF } & 11 / 21 / 03 & 11 / 20 / 06 & 175.00 & 25.00 \\ \text { PRGF } & 8 / 4 / 00 & 8 / 3 / 03 & 190.00 & 33.60 \\ \text { ESAF } & 4 / 26 / 96 & 4 / 25 / 99 & 149.55 & 24.93 \\ \text { ESAF } & 2 / 22 / 93 & 12 / 21 / 94 & 45.23 & 45.23 \\ \text { ESAF } & 5 / 15 / 89 & 3 / 31 / 93 & 261.40 & 216.17\end{array}$




\section{Projected Obligations to Fund}

(SDR million; based on existing use of resources and present holdings of SDRs):

Forthcoming

\begin{tabular}{|c|c|c|c|c|c|}
\hline & 2004 & 2005 & $\underline{2006}$ & 2007 & $\underline{2008}$ \\
\hline Principal & 4.75 & 4.99 & 9.21 & 6.72 & 6.72 \\
\hline Charges/interest & $\underline{0.36}$ & $\underline{1.07}$ & $\underline{1.03}$ & $\underline{0.99}$ & $\underline{0.96}$ \\
\hline Total & $\overline{5.11}$ & $\overline{6.05}$ & 10.24 & $\overline{7.71}$ & $\overline{7.68}$ \\
\hline
\end{tabular}

\section{Safeguards Assessments}

Under the Fund's safeguards assessment policy, the Central Bank of Kenya (CBK) is subject to a full safeguards assessment with respect to the PRGF Arrangement approved on November 21, 2003. The assessment was completed on October 21, 2003 and proposed specific measures to address a number of weaknesses, as reported in EVS/03/151 (paragraph 31). These measures are being monitored by staff under the present PRGF program

\section{Exchange Arrangements}

The currency of Kenya is the Kenya shilling. Kenya has adopted a unitary exchange rate structure in which the exchange rate is determined in the interbank market. The official exchange rate, which is set at the previous day's average market rate, applies only to government and government-guaranteed external debt-service payments and to government imports for which there is a specific budget allocation. The exchange rate regime is a managed float, in which the U.S. dollar is the principal intervention currency. Kenya maintains an exchange arrangement that is free of exchange restrictions and multiple currency practices. On November 3, 2004, the exchange rate was K Sh 81.2=US\$1.

\section{Article IV Consultations}

Kenya is on the 24-month cycle for Article IV consultations. The 2003 Article IV consultation was concluded on May 2, 2003 (IMF Country Report No. 03/199, July 9, 2003).

\section{FSAP Participation}

The joint IMF-World Bank Financial Sector Assessment Program (FSAP) missions took place in Nairobi in July and October, 2003. 


\section{Technical Assistance}

Department Purpose

MAE

MAE

STA

MAE

STA

STA

FAD

MAE

STA

FAD

FAD

MAE

MAE

STA

MAE

FAD

MFD
Mission on banking supervision

Mission on banking supervision

and bank restructuring

Resident expert on balance of payments statistics

Mission on banking supervision

Mission on government finance statistics

Mission on monetary statistics

Mission on improving expenditure

management and control

Mission on banking supervision

Mission on balance of payments statistics

Mission on internal audit

Mission on tax and customs administration

Mission on bank restructuring and review of deposit insurance system

Mission on review of banking conditions and systemic vulnerability

Two-year ongoing project on national Accounts

Mission on elements of a plan to deal with the nonperforming loan problem

AFRITAC East work plan mission

Banking Sector Restructuring
Time of Delivery

November 1999

February 1999

May-August 1999

July 1999

November-December 1999

January 2000

February 2000

May 2000

May-July 2000

November 2000

January 2001

June 2001

January 2002

May 2002-May 2004

July 2002

December 2002

January/February 2003 
FAD AFRITAC East/Bank mission on assessment of tracking of public expenditure management system

March 2003

STA

Government Finance Statistics (GDDS)

September 2003

MFD

Central Bank Accounting

February 2004

STA

Government Finance Statistics (GDDS)

February 2004

FAD

Custom Administration

March 2004

FAD

Tax Administration

April 2004

MFD

Banking Supervision/Deposit Insurance /Legislation

July 2004

MFD

Deposit Insurance/Legislation (with LEG)

October 2004

\section{Resident Representative}

The Fund has had a resident representative in Kenya since December 1993. Mr. Jürgen Reitmaier is the current Senior Resident Representative. 


\section{Kenya: IMF-World Bank Relations}

(As of November 11, 2004)

\section{A. Partnership in Kenya's Development Strategy}

1. Kenya's development strategy is set forth in the Investment Program for the Economic Recovery Strategy for Wealth and Employment Creation (IP-ERS), considered by the Boards of the IMF and the World Bank in May 2004. The IP-ERS draws upon the draft poverty reduction strategy paper (PRSP), prepared by the previous government following large-scale consultations with stakeholders. Key themes of the strategy are:

- Accelerating economic growth in an environment of macroeconomic stability;

- Strengthening institutions for governance, including implementation of the recently enacted Anti-Corruption and Economic Crimes Act and the Public Officer Ethics Act; strengthening security and law and order;

- Rehabilitating and expanding physical infrastructure, specifically the road network, including rural access roads and the energy infrastructure;

- Improving access to, and quality of, education and health services; and

- Improving the investment climate particularly for micro, small, and medium enterprises, and reforming the financial sector.

2. The IP-ERS is providing the framework for Bank-IMF support to Kenya's development efforts in the medium term. The government intends to use the IP-ERS to coordinate donor support in general, and has taken several steps towards strengthening coordination. It held a Consultative Group meeting in November 2003 for the first time since 1996. It has also established the Kenya Coordination Group, which meets once every two months and acts as a forum for engagement on issues of mutual interest. It created in October 2004 the National Economic and Social Council (NESC) within the Office of the President to oversee the implementation of the IP-ERS and advise the president on policies to increase growth and reduce poverty. The NESC comprises key ministers, international experts on development, and representatives of the private sector, the media, research institutes, and civil society.

3. In supporting Kenya's development strategy, the IMF leads the dialogue on monetary and exchange rate policy. The Fund coordinates with the Bank on fiscal policy and structural reforms in the areas of public expenditure management, governance, financial sector, revenue administration, trade, and the public wage bill. The Bank takes the lead on monitoring implementation of the IP-ERS, dialogue in the areas of infrastructure, human development, private sector development, rural development, and reforms of public enterprises and the civil service. In analytic work, the Bank takes the lead on growth and poverty analysis while the two coordinate on trade, labor markets, and poverty and social impact analysis. 


\section{B. Bank Group Strategy}

4. A new country assistance strategy (CAS) of reengagement for fiscal 2004-07 was approved by the World Bank Board on June 17, 2004. The CAS is closely aligned with the development priorities set forth in the IP-ERS. A key challenge will be to build on the IPERS as the basis for much greater harmonization of donor programs, while shifting assistance away from projects and toward sectorwide programs and budget support. Increasing levels of budget support will require the development of a positive record of reform through the implementation of Bank and IMF-supported reform programs, specifically:

- Improvements in the public accountability framework, specifically in the areas of public expenditure management and procurement.

- Development of a strong participatory monitoring and evaluation system to track implementation progress and impact of the IP-ERS objectives.

- $\quad$ Pro-poor shifts in public expenditure patterns.

- $\quad$ Undertaking of necessary analytical work, such as the poverty and social impact analysis of planned policy reforms.

The Bank's new strategy has been formulated to promote in a carefully sequenced way growth and poverty reduction. Project lending in sectors with sound policies and strategies (for example transport and energy) will help to further strengthen institutions and financing mechanisms that deepen and nurture good governance. Policy-based lending will support critical reforms in public sector management and the financial sector. Technical assistance projects will develop, and assist in implementing, public sector reforms, privatization, and legal and financial sector reforms. In line with the Bank's commitment to donor harmonization, subsequent interventions will support sectorwide approaches in health and education, and budget support for implementation of the IP-ERS through a series of annual poverty reduction support credits (PRSCs).

5. Economic and public sector management. The IP-ERS gives high priority to budgetary and public expenditure reforms. The Bank is preparing an Economic Recovery Strategy Support Credit (ERSSC) to support government actions to strengthen budgetary and public financial management, improve governance, enhance agricultural productivity, and to improve the climate for private investment. The goal of the ERSSC is to lay the foundation of a medium-term development program that could be supported through annual budget support. The Bank is also providing technical assistance to the government for its annual PER process with a view to strengthening budget management and strategic resource allocation and fostering greater openness and participation in budget matters. A Public Sector Management Technical Assistance Project (PSMTAP) for US\$15 million approved in July 2001 and due to close on June 30, 2005 is providing support for civil service reform and capacity building, internal auditing, annual medium-term expenditure framework (MTEF) 
process, implementation of the integrated financial management information system (IFMIS), and development of improved procurement practices. A follow-on to the PSMTAP is planned for fiscal 2006 to further build capacity for budget and financial management, tax administration, and support reforms of parastatals. To strengthen governance, this project will also support training on regulatory and other issues, assist with the preparation of plans for corporate restructuring and privatization, strengthen the capacity and design of regulatory bodies, and improve consultative mechanisms between public and private actors.

6. To enhance the capacity of the Central Bureau of Statistics and other agencies, the Bank has prepared a Statistical Capacity-Building Project (StatCap) scheduled for Board presentation during fiscal 2005. The Bank is helping to build capacity for monitoring and evaluation through a grant from the Institutional Development Fund. The Bank is also supporting government efforts to analyze policy and spending choices through the grantsupported Poverty and Data Initiative and related analytical work on poverty.

7. Financial sector development. The IP-ERS identifies the urgent need to reform the financial sector in Kenya, which is beset with a high level of nonperforming loans and wide interest rate spreads, and operates within a weak legal and judicial system. The government is considering divesting the state's stake in two of the biggest banks. To support the government's reform agenda, the Bank and the IMF have recently concluded a joint Financial Sector Assessment Program that significantly deepened knowledge of the issues facing the financial sector. To support reforms, the Bank prepared a Financial and Legal Sector Technical Assistance Credit that was approved by the Board in October 2004. This will support the development of financial and legal sector strategies, the strengthening of financial and legal sector regulations and institutions, and the establishment of a bank restructuring and privatization project within the Ministry of Finance that will guide the process of privatizing banks. This will lay the foundation for a Financial Sector Adjustment Credit (FSAC), a budget support operation planned for fiscal 2005, that will support policy and institutional reforms, including divestiture of the state's remaining ownership stakes in the banks, enhanced banking supervision, and design of a policy to expand access to financial services, including in rural areas.

8. Privatization and private sector participation. Bank activity in this area has aimed at providing technical advice toward developing and implementing strategies for private sector participation in major public utilities. With the help of grants from the Public-Private Infrastructure Advisory Facility (PPIAF), the government has completed a study on options for concessioning of trunk roads and a study on the legal and regulatory framework for the transport sector. The Northern Corridor Transport Improvement Project, which became effective in September 2004, will facilitate private sector participation in the financing, construction and management of the road. The IFC is the transaction adviser for concessioning the Kenya Railways jointly with Uganda Railways. Public-private partnerships are also being considered to provide water and energy services.

9. Private sector development. A joint Bank and IFC supported Micro, Small and Medium Enterprise Project aimed at facilitating the creation and growth of MSMEs was 
approved by the Board in July 2004. A three-year pilot Small and Medium Enterprises Solutions Center is planned for fiscal 2005. In addition, the IFC is supporting the multidonor Africa Project Development Facility that facilitates firms' access to financial and other services, and the African Management Services Company that provides management expertise. Two recently completed studies - the investment climate assessment and the administrative barriers study conducted by the Bank Group's Foreign Investment Advisory Service (a joint IFC and Bank program)-have provided a greater understanding of the obstacles to private sector development in Kenya and the policies needed to address them. The ERSSC planned for Board approval in fiscal 2006 will support implementation of the recommendations of the study.

10. Infrastructure development. The IP-ERS recognizes the lack of adequate physical infrastructure as a major impediment to private sector development in Kenya. The Bank is supporting the government's program through its Northern Corridor Transport Improvement Project, which will finance the reconstruction of the road network that links Kenya with Uganda, Rwanda, Burundi, and the Democratic Republic of Congo. The project will also help to upgrade Nairobi's Jomo Kenyatta International Airport to address the threat of terrorism. The IFC is serving as the transactions advisor for the joint concessioning of Kenya's and Uganda's national railways, expected to be completed by summer 2005. Improving management of the railways is expected to be a major catalyst to regional integration and growth. The grant-financed Nairobi Water and Sewerage Institutional Restructuring Project, which became effective in September 2004, aims at building a strong institutional and governance framework that will promote efficient and sustainable delivery of water and sewerage services in Nairobi. The Energy Sector Recovery Project, effective in November 2004, will help to improve the efficiency of production and distribution of electricity, to promote private sector participation in the sector, and to improve power sector management and corporate governance.

11. Agriculture and environment. The Bank is supporting measures to increase agricultural productivity and competitiveness in several ways. Through an analysis of agricultural markets and rural service delivery, an analysis of the linkages between poverty and agricultural growth, and studies on cotton, coffee, sugar, pyrethrum, and horticulture, the Bank along with other partners is contributing to knowledge that will add in a substantial way to the policy dialogue with government. The ERSSC and the subsequent PRSCs will support implementation of the reforms. The Agricultural Productivity Project, which became effective in October 2004, is supporting the transformation of Kenya's research and extension system to a more demand driven, farmer-led and pluralistic system, while supporting associated policy reforms. The Arid Lands Resource Management Project Phase Two, effective in September 2003, is using a multisectoral, district based, community driven development approach to reduce poverty in the arid and semiarid districts of Kenya. The multicountry Lake Victoria Environmental Project is helping to establish institutions for the sustainable management of the lake and its catchment area. The GEF-supported Western Kenya Integrated Ecosystem Management Project, to be presented to the Board in January 2005, will seek to improve management of soils, water, and biodiversity, while raising the productivity of agriculture. A GEF-supported Agriculture Productivity and Sustainable Land 
Management Project, scheduled for Board presentation in May 2005 will promote improved soil and water management of key water catchments. A Bank water resources sector memorandum is preparing the ground for a future project focused on improving management of water resources.

12. Education and health. In education, the Bank is supporting the government's strategy through the Free Primary Education Project (US\$50 million grant) (effective in July 2003), through recently completed analytical work on primary and secondary education, and through support to the ministerial PER. It is now preparing a sectorwide (SWAp) education program for approval in fiscal 2006 to support both primary and secondary education. This will lay the groundwork for future support to the sector through a PRSC by strengthening monitoring and evaluation, financial management, and the planning and allocation of expenditures. The evolution in the sector will also require expanded, more detailed sectoral expenditure reviews, as alignment of expenditures with the strategy will be a key determinant of partner support for the sector. The grant-financed Global Development Learning Network project effective in February 2004 is helping to develop enhanced mechanisms of learning and knowledge-sharing among policy makers. In health, the Bank is supporting the government's strategy through analytical work focusing on a pharmaceutical sector review (fiscal 2005), and on a comprehensive health sector PER. The ongoing Decentralized AIDS and Reproductive Health Project seeks to improve mother and child health through more integrated delivery of child survival, reproductive health, and HIV/AIDS services. This operation is preparing the ground for the Bank's support to the health sector through a SWAp in fiscal 2006 that will be developed in partnership with all major stakeholders in the sector.

13. HIV/AIDS. The Bank is vigorously supporting the government's program to control HIV/AIDS. Kenya was the first country to access funds under the Africa Region's MultiCountry AIDS Program (MAP). A follow-on MAP is planned for fiscal 2006 that will focus on prevention while also supporting new initiatives to reach the vulnerable groups and to increase access by those already infected to comprehensive care and treatment. The Bank supports the National AIDS Control Council and its provincial, district, and local-level bodies and line ministries in the mainstreaming of HIV/AIDS-related activities.

14. Nonlending activities. The Bank completed a Strategic Gender Assessment in fiscal 2003 that reveals the high poverty and vulnerability of many Kenyan women and describes the discrimination that girls and women face in gaining access to health and education services, and ultimately to employment opportunities. Analytical work planned for the next two or so fiscal years is designed to: (a) support efficient public allocation of funds to priority development areas, (b) expand on the analytical work already initiated in fiscal 2003 on poverty analysis, particularly rural poverty; (c) expand on the findings of the 2003 country economic memorandum to better understand the sources of growth and competitiveness, (d) support growth in agricultural and livestock production, (e) better understand barriers to trade, and (g) deepen knowledge of labor markets. In preparation for the budget support credits, core diagnostic economic and sector work is planned for fiscal 2005, including a country procurement assessment report, an updated country financial accountability assessment, and a poverty analysis. 


\section{Summary of Bank's Lending Portfolio}

(As of October 31, 2004)

\begin{tabular}{|c|c|c|c|c|}
\hline Project & Sector & $\begin{array}{l}\text { Closin } \\
\text { g Date }\end{array}$ & $\begin{array}{l}\text { /Credit } \\
\text { unt } \\
\text { hillions } \\
\mathrm{S} \\
\mathrm{rs})\end{array}$ & $\begin{array}{l}\text { Undisburs } \\
\text { ed on } \\
10 / 31 / 04\end{array}$ \\
\hline Urban Transport Infrastructure & Transport & $6 / 05$ & 115.00 & 25.78 \\
\hline Development Learning Center & Public Admin & $12 / 08$ & 2.7 & 2.42 \\
\hline HIV/AIDS Disaster Response & Health & $6 / 05$ & 50.00 & 20.13 \\
\hline $\begin{array}{l}\text { Decentralized AIDS and Reproductive } \\
\text { Health Project }\end{array}$ & Health & $6 / 05$ & 50.00 & 33.85 \\
\hline Regional Trade Facilitation Project & Private Sector & $6 / 11$ & 25.00 & 14.78 \\
\hline Public Sector Management TA Program & Public Sector & 06/05 & 15.00 & 6.34 \\
\hline Arid Lands II & Agriculture & $6 / 09$ & 60.00 & 53.47 \\
\hline Free Primary Education & Education & $12 / 06$ & 50.00 & 6.64 \\
\hline Lake Victoria Environment (GEF) & Environment & $12 / 05$ & 11.50 & 4.66 \\
\hline Nairobi Water \& Sewerage TA & Water & 06/07 & 15.00 & 13.69 \\
\hline Agricultural Productivity & Agriculture & $12 / 07$ & 40.00 & 39.79 \\
\hline Northern Corridor Transport & Transport & $12 / 09$ & 207.00 & 196.28 \\
\hline Energy Sector Recovery & Energy & $03 / 10$ & 80.00 & 80.64 \\
\hline Financial and Legal Technical Assistance & Finance & $03 / 10$ & 18.00 & 17.90 \\
\hline MSME Competitiveness & Private Sector & $06 / 10$ & 22.00 & 21.91 \\
\hline Total Active $=15$ & & & 761.20 & 538.28 \\
\hline
\end{tabular}

\section{IMF-World Bank Collaboration in Specific Areas}

15. The IMF and World Bank staffs maintain a close collaborative relationship in supporting structural reforms in Kenya through lending, analytic work, and technical assistance.

16. Joint assessment of the government's IP-ERS. The staffs of the Bank and the IMF jointly assessed Kenya's IP-ERS in April 2004. These documents were discussed by the Bank Board on May 6, 2004 and by the IMF Board on May 10, 2004.

17. Macroeconomic framework. The IMF leads the analysis and dialogue on monetary policy, which serves as input into the Bank program. The monetary policy dialogue focuses on issues related to quantitative monetary targets, such as money supply and inflation, exchange rate changes, and central bank operations. The Bank collaborates with the IMF on fiscal policy dialogue, which broadly covers issues related to fiscal sustainability and size of the public sector. Currently, the dialogue focuses on the size of overall fiscal deficit, fiscal risks, public service wage bill, pay reform, revenue performance, including tax 
administration and adjustment to tax policy, and domestic debt financing of the deficit. The Bank takes the lead on the analysis of issues related to economic growth and poverty. The Bank and the IMF are also collaborating on trade reform.

18. Budgetary and public expenditure reforms. The Bank and IMF work closely to support government efforts in budgetary and public expenditure reforms. The Bank leads the dialogue on planning, the preparation of the MTEF, strategic resource allocation (particularly aligning budgetary allocations with priorities laid out in the IP-ERS), expenditure restructuring, enhanced stakeholder participation, and issues related to efficiency of public spending, such as expenditure tracking. A joint Bank-IMF Public Expenditure Management Assessment and Action Plan (PEM-AAP) was completed in April 2004. A technical working group that includes Bank and IMF representatives, along with other donors and stakeholders, monitors the PER process and implementation of the reform program. The Bank and IMF collaborate on issues related to internal audit reform and on the IFMIS. The Bank and the IMF are members of the high-level Budget Support Donor Coordination Group and the technical-level Joint Technical Working Group that are helping the government to prepare for budget support. The Bank and the IMF are coordinating closely on the ERSSC conditionality and that under the PRGF arrangement.

19. Strengthening revenue collection and administration. The Bank and the Fund jointly have recently carried out detailed assessments of tax administration and customs operations. The Fund has been providing technical assistance to the Kenya Revenue Authority. The Bank has been assisting the customs department carry out a study of the length of time it takes to clear a container through customs. The Fund (through AFRITAC) and the Bank have been collaborating closely in supporting the Kenya Revenue Authority to prepare a reform and modernization plan for funding under the follow-on PSMTAP planned for fiscal 2006.

20. Debt management and debt sustainability analysis. The IMF has recently updated its external debt sustainability analysis. In the context of the Financial Sector Assessment Program, a joint World Bank/IMF initiative is being undertaken to support capacity-building in the area of sovereign debt management and domestic debt market development.

21. Governance reforms. High levels of public corruption and a poor security situation contributed to Kenya's poor economic performance in the last decade, and governance reforms are a key element of the government's strategy to promote economic growth and reduce poverty. The new PRGF arrangement itself will support a deepening of anticorruption measures. The Bank is supporting the strengthening of legal and judicial institutions through the Financial and Legal Sector Technical Assistance Project (see paragraph 7). The Bank in 2005 will prepare a country procurement assessment report in fiscal 2005 jointly with the government.

22. Financial sector reforms. The IMF and Bank are collaborating on financial sector reforms in several ways. They have conducted a joint in-depth assessment of the financial sector (banking, insurance, and capital markets) through the Financial Sector Assessment 
Program being led by the IMF (see paragraph 7). The Financial Sector Assessment Program has produced technical notes on selected topics, detailed assessments of standards and codes, the IMF's Financial System Stability Assessment, and the Bank's Financial Sector Assessment. The Bank and the IMF are expected to coordinate closely on the prior actions and conditionality of the FSAC.

23. Privatization and private sector development. The dialogue on privatization and regulatory reforms is led by the Bank, and the IMF is a partner in this policy dialogue, particularly on issues of the fiscal burden. The Bank is supporting growth of private sector participation through regulatory reform and through assistance for privatization or restructuring of public enterprises in power, railways (IFC), and urban water. MIGA is supporting private sector development through international comparisons of competitiveness, through the provision of technical assistance and capacity building services to the country's investment promotion intermediaries, and through the offer of political risk guarantees to foreign investors going into the country.

24. Trade reforms and regional integration. Trade issues and regional integration are prominent within the government's policymaking priorities. Kenya is expected to form a customs union with Tanzania and Uganda in 2005 in the context of the East African Community (EAC). Kenya is also actively involved in negotiations and activities of the Common Market for Eastern and Southern Africa (COMESA). The IMF and the Bank both assist with reform of the tariff regime, and the Bank is providing technical advice on regional integration issues. The Bank recently produced a technical note on the implications of an EAC customs union for revenue and trade flows. The note is currently being updated to account for the latest agreement on the common external tariff. The IMF served as a peer reviewer for the note. The Bank is undertaking a diagnostic trade integration study, to be completed in fiscal 2005. The Bank is undertaking an assessment of nonfiscal investment incentives in each of the three states of the EAC. This work is complemented by a recently completed IMF assessment of fiscal investment incentives. Both assessments will be used to harmonize such incentives in the context of the planned customs union. The Bank is implementing a Regional Trade Facilitation Project (US\$25 million) aimed at improving access to finance for cross-border trade between participating countries. A follow-on project is planned for fiscal 2006. The Bank is also preparing an EAC Regional Transport and Trade Facilitation Project for fiscal 2006. The project includes support for implementation of the customs union and for institutional and investment support to facilitate transport in the region.

Prepared by World Bank staff. Questions may be addressed to Makhtar Diop, Country Director, Kenya, at 5368+3441, or Praveen Kumar, Country Economist, at X-36347. 


\section{Kenya: Statistical Issues}

1. Weaknesses in key macroeconomic statistics hamper economic analysis and surveillance. Kenya's macroeconomic statistics have deteriorated significantly over the past decade, reflecting primarily managerial and organizational weaknesses, and inadequate resources provided to the Central Bureau of Statistics (CBS). During the 2003 Article IV consultation discussions, the staff emphasized the need to improve the timeliness and quality of these statistics, and encouraged the authorities to promptly implement STA recommendations from previous technical assistance..

2. The authorities are taking actions, with donor assistance, to improve the quality and timeliness of economic and financial statistics. Kenya participates in the General Data Dissemination System (GDDS) and the GDDS project for Anglophone Africa; metadata and detailed plans for improving the data over the short and medium term have been posted on the Fund's Dissemination Standards Bulletin Board (DSBB). Kenya received Fund technical assistance in the context of this project, and further assessment of capacity building requirements has been undertaken by the AFRITAC East Statistical Advisor. Furthermore, the authorities are preparing a statistical development plan and a new statistical act to strengthen the independence of the CBS.

3. A STA multitopic mission to Kenya in 1998 undertook a comprehensive review of the major statistical areas and prepared a work program for implementation of its principal recommendations, to address most of the problems discussed below. A peripatetic STA advisor assisted the authorities in improving the balance of payments statistics and the national accounts data.

4. Monetary, exchange rate, and some external data are published on a monthly and biannual basis by the Central Bank of Kenya (CBK) in its Monthly Economic Review. Core financial data are also made available to the Fund on a regular basis. A detailed account of various sectoral activities and the corresponding statistical data are published annually by the CBS in its Economic Survey.

\section{National accounts}

5. Data quality, which was previously reported as good, has deteriorated significantly because of budgetary and staff constraints at the CBS. GDP is believed to be significantly underestimated, as important and increasing parts of the economy, such as the informal sector, nonagriculture subsistence, horticulture, and self-employed professionals are not properly covered.. The STA peripatetic advisor assisted the authorities to rebase the national accounts estimates at constant 2001 prices and compile institutional accounts for the general government sector. In May 2004, revised national accounts estimates following the 1993 SNA (at constant 2001 prices) for the years 1996-2003 were released. 


\section{Prices and production}

6. The CBS commenced publication in early 2002 of a new national CPI (covering 12 urban areas), with a base year of 1997 and weights derived from the 1992-93 HBS. The index is compiled and published on a timely basis. There is no producer price index, or any short-term export and import price indices produced.

\section{Government finance statistics}

7. The 1998 multisector statistics mission found several statistical problems with the cumulative year-to-date monthly and quarterly fiscal data that the Ministry of Finance (MoF) compile and report to the AFR for budget-monitoring purposes. These data, which do not conform to the Fund's government finance statistics (GFS) methodology, do not adequately facilitate economic analysis, mainly because of inappropriate classification of expenditure data. The MoF has not developed a system to adequately monitor expenditure commitments, or domestic arrears, and relies mainly on monitoring cash movements in government accounts at the CBK. The recording of external financing and expenditure directly financed from abroad also remains an area for improvement. The 1998 mission made several recommendations for improving the data, most of which have not been implemented.

8. The CBS compiles and reports to the STA aggregate annual GFS revenue and expenditure data for budgetary central government based on detailed data in the reports of the Controller and Auditor General, using a methodology established by a 1999 STA GFS mission. Lack of proper computerization prevents the CBS from compiling more detailed GFS data. Considerable differences exist between these data and the data compiled by the $\mathrm{MoF}$ and reported to AFR for surveillance and program monitoring purposes.

9. If implemented, the recommendations of the February 2004 technical assistance mission in GFS will significantly improve the quality and availability of fiscal data.

\section{Monetary statistics}

10. Monetary statistics are compiled from a bank reporting system and are broadly adequate for policy, analytical, and supervisory purposes. A STA technical assistance mission in January 2000 observed that the authorities had implemented virtually all recommendations of the previous STA missions, which had led to a number of improvements in Kenya's monetary statistics. However, the mission identified and made recommendations to address further problems in monetary data, in particular (a) asymmetrical interbank positions, (b) the statistical implications on the data of numerous bank closures, and (c) the coverage of international reserves. Since then, the authorities have initiated revisions to monetary statistics in line with the recommendations of the mission.

\section{Balance of payments statistics}

11. The CBS compiles and reports balance of payments data in Kenya shillings annually 
on a regular basis to STA, although with a considerable lag. In addition, the CBK compiles a complete set of annual balance of payments statistics in U.S. dollars, which are reported to AFR and used for programming and surveillance purposes. The two datasets are not entirely consistent, and Fund staff has been strongly encouraging the authorities to reconcile them. More recently, the CBK also started to compile and publish discrete quarterly balance of payments estimates.

12. The quality of the balance of payments data has deteriorated. Although the overall quality of trade data may be reasonably good, data for other current account and many financial account transactions are rather weak. Following the liberalization of the exchange system in 1993-94, gaps have emerged in the coverage of balance of payments source statistics. The compilation system (other than that used for compiling customs statistics), used since 1994, relies on reports from domestic banks and may result in a substantial underrecording of current earnings, including tourism receipts, as well as a failure to capture transactions that are settled via accounts held abroad.

13. Present estimates of direct and portfolio investment are believed to be substantially understated. The large positive errors and omissions in the central bank data that have emerged in the balance of payments since 1994 raise concerns as to the potential size of external obligations. The MoF compiles data covering Kenya's public and publicly guaranteed external obligations to official and commercial creditors. This database does not take account of nonresident purchases of the government's domestic currency-denominated debt securities.

\section{External debt statistics}

14. In developing the loan-by-loan debt sustainability analysis (DSA) in 2002, Fund and World Bank staff identified several significant debt data problems that have since been addressed by the authorities. Nevertheless, significant debt data management problems remain, along with more general issues in the area of external debt management and its integration in the budget formulation and expenditure management systems. 
Kenya: Survey of Reporting of Main Statistical Indicators

\begin{tabular}{|c|c|c|c|c|c|c|c|c|c|c|c|c|}
\hline & $\begin{array}{c}\text { Exchange } \\
\text { Rates } \\
\end{array}$ & $\begin{array}{c}\text { Central } \\
\text { Bank } \\
\text { Balance Sheet }\end{array}$ & $\begin{array}{c}\text { International } \\
\text { Reserves }\end{array}$ & $\begin{array}{c}\text { Reserve/ } \\
\text { Base Money }\end{array}$ & $\begin{array}{l}\text { Broad } \\
\text { Money }\end{array}$ & $\begin{array}{c}\text { Interest } \\
\text { Rates }\end{array}$ & $\begin{array}{c}\text { Consumer } \\
\text { Price } \\
\text { Index } \\
\end{array}$ & $\begin{array}{c}\text { Central } \\
\text { Government } \\
\text { Balance }\end{array}$ & $\begin{array}{l}\text { Exports/ } \\
\text { Imports }\end{array}$ & $\begin{array}{l}\text { Current } \\
\text { Account } \\
\text { Balance }\end{array}$ & $\begin{array}{c}\text { External } \\
\text { Debt }\end{array}$ & $\begin{array}{l}\text { GDP/ } \\
\text { GNP }\end{array}$ \\
\hline $\begin{array}{l}\text { Date of latest } \\
\text { observation }\end{array}$ & $7 / 31 / 04$ & $8 / 30 / 04$ & $7 / 31 / 04$ & $8 / 30 / 04$ & $8 / 30 / 04$ & $8 / 30 / 04$ & $9 / 2004$ & $6 / 2004$ & $3 / 2004$ & 2003 & $3 / 2004$ & \\
\hline Date received & $8 / 6 / 04$ & $10 / 8 / 04$ & $8 / 6 / 04$ & $10 / 8 / 04$ & $10 / 8 / 04$ & $10 / 8 / 04$ & $10 / 2004$ & $8 / 16 / 2004$ & $6 / 2004$ & 8/30/04 & $8 / 31 / 2004$ & t8/2004 \\
\hline $\begin{array}{l}\text { Frequency } \\
\text { of data }{ }^{1}\end{array}$ & $\mathrm{D}$ & $\mathrm{D}$ & $\mathrm{D}$ & $\mathrm{D}$ & M & W & M & M & M & A & A & A \\
\hline $\begin{array}{l}\text { Frequency } \\
\text { of reporting }\end{array}$ & $\mathrm{D} / \mathrm{W}$ & $\mathrm{D} / \mathrm{W}$ & $\mathrm{D} / \mathrm{W}$ & $\mathrm{D} / \mathrm{W}$ & M & W/M & M & M & M & A & A & A \\
\hline $\begin{array}{l}\text { Frequency of } \\
\text { publication }^{1}\end{array}$ & $\mathrm{D}$ & $\mathrm{M}$ & $\mathrm{M}$ & M & M & $\mathrm{D}$ & M & Q & M & M & $\mathrm{A}$ & A \\
\hline $\begin{array}{l}\text { Source of } \\
\text { data }^{2}\end{array}$ & DR & DR & DR & DR & DR & DR & DR & DR & DR & DR & DR & OP \\
\hline $\begin{array}{l}\text { Mode of } \\
\text { reporting }\end{array}$ & $\mathrm{F}$ & $\mathrm{F}$ & $\mathrm{F}$ & $\mathrm{F}$ & $\mathrm{F}$ & $\mathrm{F}$ & $\mathrm{F}$ & $\mathrm{F}$ & $\mathrm{F} / \mathrm{P}$ & $\mathrm{P} / \mathrm{SV}$ & $\mathrm{P} / \mathrm{SV}$ & $\mathrm{P} / \mathrm{SV}$ \\
\hline Confidentiality ${ }^{4}$ & $\mathrm{E}$ & $\mathrm{R}$ & $\mathrm{R}$ & $\mathrm{R}$ & E & E & E & $\mathrm{R}$ & E & E & E & E \\
\hline
\end{tabular}




\section{Kenya: Debt Sustainability Analysis Update}

This annex updates the debt sustainability analysis conducted as part of the last staff report for Kenya IMF Country Report No. 03/400, January 8, 2004 and IMF Country Report No. 03/399, January 8, 2004. The broad conclusions of the previous exercise, that the debt is sustainable (taking into account the impact of a Paris Club rescheduling on external debt), remain valid; under the revised program framework, external and central government debt sustainability improves.

\section{Central Government Debt Sustainability}

The updated DSA for central government debt continues to show a steady decline in central government debt (Table 1). In 2004 and 2005, the ratio is actually lower under the present scenario than under the baseline presented in November 2003, reflecting the underperformance of domestic borrowing and of donor inflows in 2003/04, as well as expectations that donor inflows will be lower throughout the program period than projected in the earlier DSA. The fact that in the medium/long-term (2007 to 2015), the ratio is not even one full percentage point higher under the updated analysis compared to the earlier DSA indicates that the projected increase in domestic borrowing in the revised program scenario does not materially jeopardize debt sustainability. Kenya's central government debt profile appears relatively robust to various shocks. The most serious stress would come from a sharp real depreciation; under this alternative scenario, the NPV debt to GDP ratio increases to around 71 percent during the program period.

\section{External Debt Sustainability}

Under the revised program scenario, the NPV of debt to exports ratio is lower than under the original DSA, averaging 104 percent over the period 2004-07 versus 120 percent over the same period in the November 2003 analysis. This lower debt profile reflects several factors. One is that the expectations of donor inflows did not materialize in the first year of the program (as detailed in the staff report) and projections of expected inflows have been lowered. A second factor is that since the last DSA, Kenya secured a rescheduling with the Paris Club in January 2004, and while the Houston terms rescheduling does not lower the debt stock, it will reduce debt service paid during the 2004-2007 consolidation period. A technical factor to be kept in mind is that the DSA is carried out for official debt only, due to the unavailability of data on the private sector external debt stock; under the macroeconomic framework in the staff report, it is expected that as the country reaps the benefits of reforms in terms of growth and an improved investment climate, external loans to the private sector would increase, and would add to the relatively moderate ratio of NPV of debt to GDP that is projected in this analysis. Finally, the debt data weaknesses that Kenya has continued to experience mean that DSA results must be interpreted with caution. An on-going project supported by, inter alia, a World Bank Financial Sector and Legal Technical Assistance credit, is expected to produce significantly strengthened debt data, and an updated DSA will be conducted when these data are available. 
A combined real GDP growth and net non-debt creating low shock has the largest impact on the NPV debt to exports ratio, pushing the indicator to 173 percent in 2005; however, the ratio declines relatively quickly to below 150 percent.

The debt service due ratio averages 8 percent during the program period, and declines further over the long-term. Alternative scenarios indicate that the indicator would increase significantly, albeit generally not to levels of concern, in response to most of the stresses tested in the sensitivity analyses. The debt service ratio is most affected by a fall in export value growth and the combined GDP growth and non-debt creating flow shock.

\section{Conclusions}

Kenya's debt appears sustainable under the current scenario, but the fact that certain shocks have the potential to push debt indicators to higher levels means that the authorities must remain vigilant. The authorities have stated that fiscal and external debt management policies will continue to balance the commitment to debt sustainability with the need to support the poverty reduction strategy. 
Table 1. Kenya: Central Government Debt Sustainability Framework, Before Rescheduling, 2003-2015 (In percent of GDP, unless otherwise indicated)

\begin{tabular}{|c|c|c|c|c|c|c|c|c|c|}
\hline & \multicolumn{2}{|c|}{ Actual } & \multicolumn{7}{|c|}{ Projections } \\
\hline & 2001 & 2002 & 2003 & 2004 & 2005 & 2006 & 2007 & 2010 & 2015 \\
\hline & & & \multicolumn{7}{|c|}{ I. Baseline Projections } \\
\hline Total central government debt & 51.5 & 53.6 & 51.1 & 49.7 & 49.4 & 48.8 & 47.3 & 44.6 & 39.9 \\
\hline Foreign-currency denominated debt 1 / & 30.7 & 30.1 & 27.8 & 27.4 & 27.2 & 27.1 & 26.7 & 23.3 & 18.6 \\
\hline Domestic currency denominated debt & 20.9 & 23.5 & 23.3 & 22.3 & 22.1 & 21.7 & 20.6 & 21.3 & 21.3 \\
\hline Change in NPV & -6.1 & 2.0 & -2.5 & -1.4 & -0.3 & -0.5 & -1.5 & -3.9 & -0.8 \\
\hline Identified debt-creating flows & -1.3 & 0.8 & -4.8 & -2.9 & -3.2 & -1.2 & -0.5 & -0.7 & 0.3 \\
\hline Primary deficit & -1.9 & 0.4 & -1.1 & -1.2 & 0.1 & 0.7 & 0.4 & 0.5 & 0.5 \\
\hline Revenue and grants & 24.1 & 21.9 & 22.6 & 22.7 & 23.7 & 25.2 & 25.7 & 19.9 & 19.9 \\
\hline of which: grants & 2.2 & 0.8 & 2.0 & 1.5 & 2.8 & 3.7 & 3.2 & 1.5 & 1.5 \\
\hline Primary (noninterest) expenditure & 22.2 & 22.3 & 21.6 & 21.5 & 23.8 & 25.9 & 26.1 & 20.4 & 20.4 \\
\hline Grant element of new concessional loans & 0.0 & 0.0 & -2.0 & -2.3 & -3.3 & -3.2 & -2.4 & -2.1 & -1.4 \\
\hline Automatic debt dynamics & 0.6 & 0.5 & -1.8 & 0.5 & -0.1 & 1.3 & 1.5 & 0.9 & 1.2 \\
\hline of which: contribution from real discount rate on foreign-currency debt & 2.7 & 2.7 & 2.5 & 2.4 & 2.2 & 2.1 & 2.0 & 1.8 & 1.4 \\
\hline of which : contribution from real interest rate on domestic debt & 1.1 & 1.1 & 0.0 & 0.0 & 0.7 & 1.3 & 1.5 & 1.7 & 1.9 \\
\hline of which: contribution from real GDP growth & -0.6 & -0.5 & -0.9 & -1.2 & -1.5 & -1.6 & -1.8 & -2.3 & -1.9 \\
\hline of which: contribution from real exchange rate depreciation & -2.6 & -2.7 & -3.3 & -0.7 & -1.5 & -0.5 & -0.2 & -0.3 & -0.3 \\
\hline Other identified debt-creating flows & 0.0 & -0.1 & 0.0 & 0.0 & 0.0 & 0.0 & 0.0 & 0.0 & 0.0 \\
\hline Privatization receipts (negative) & 0.0 & -0.1 & 0.0 & 0.0 & 0.0 & 0.0 & 0.0 & 0.0 & 0.0 \\
\hline Recognition of implicit or contingent liabilities & 0.0 & 0.0 & 0.0 & 0.0 & 0.0 & 0.0 & 0.0 & 0.0 & 0.0 \\
\hline Debt relief (HIPC and other) & 0.0 & 0.0 & 0.0 & 0.0 & 0.0 & 0.0 & 0.0 & 0.0 & 0.0 \\
\hline Other (specify, e.g. bank recapitalization) & 0.0 & 0.0 & 0.0 & 0.0 & 0.0 & 0.0 & 0.0 & 0.0 & 0.0 \\
\hline Residual, including asset changes & -4.8 & 1.2 & 2.3 & 1.6 & 2.9 & 0.7 & -1.1 & -3.2 & -1.0 \\
\hline \multicolumn{10}{|l|}{ Other Sustainability Indicators } \\
\hline Nominal value of Central Government debt & 65.3 & 65.7 & 59.5 & 55.6 & 54.6 & 53.6 & 52.0 & 48.7 & 41.2 \\
\hline NPV of contingent liabilities (not yet officially recognized in Central Government debt) & 0.0 & 0.0 & 0.0 & 0.0 & 0.0 & 0.0 & 0.0 & 0.0 & 0.0 \\
\hline Gross financing need $2 /$ & 13.4 & 13.9 & 9.5 & 8.1 & 9.1 & 9.6 & 9.3 & 3.5 & 3.3 \\
\hline Central Government debt-to-revenue-and-grants ratio (in percent) & 271 & 299 & 263 & 245 & 231 & 212 & 202 & 245 & 207 \\
\hline Debt service-to-revenue-and-grants ratio (in percent) 3 / & 60.0 & 65.4 & 47.9 & 41.3 & 37.6 & 35.5 & 34.5 & 30.1 & 28.7 \\
\hline Primary deficit that stabilizes the NPV of debt-to-GDP ratio & 4.2 & -1.7 & 1.4 & 0.2 & 0.5 & 1.2 & 1.9 & 4.4 & 1.3 \\
\hline \multicolumn{10}{|l|}{ Key Macroeconomic and Fiscal Assumptions } \\
\hline Real GDP growth (in percent) & 1.1 & 1.1 & 1.7 & 2.4 & 3.0 & 3.4 & 3.8 & 5.0 & 5.0 \\
\hline Average nominal interest rate on forex debt (in percent) & 2.1 & 1.8 & 1.7 & 1.9 & 1.9 & 1.9 & 1.9 & 1.9 & 1.8 \\
\hline Average real interest rate on domestic debt (in percent) & 5.6 & 5.5 & -0.1 & 0.2 & 3.3 & 6.1 & 7.0 & 7.4 & 9.6 \\
\hline Real exchange rate depreciation (percentage change, $+=$ depreciation) & -6.7 & -8.2 & -10.4 & -2.3 & -5.2 & -1.7 & -0.7 & -1.3 & -1.3 \\
\hline Inflation rate (GDP deflator, in percent) & 9.1 & 8.4 & 11.5 & 10.3 & 7.8 & 4.4 & 3.6 & 3.3 & 3.3 \\
\hline US Inflation rate (GDP deflator, in percent) & 2.4 & 1.1 & 1.5 & 1.2 & 1.7 & 2.0 & 2.0 & 2.0 & 2.0 \\
\hline Growth of real primary spending (deflated by GDP deflator, in percent) & 2.8 & 1.5 & -1.7 & 1.9 & 14.2 & 12.6 & 4.6 & 5.0 & 5.0 \\
\hline \multirow[t]{2}{*}{ Major commodity price (tea) } & 1.6 & 1.6 & 1.7 & 1.8 & 1.8 & 1.9 & 1.9 & 1.9 & 1.9 \\
\hline & & & \multicolumn{7}{|c|}{ II. Stress Tests for NPV } \\
\hline \multicolumn{10}{|l|}{ Alternative Scenarios } \\
\hline A1. Real GDP growth and primary balance are at historical averages 4/ & & & 50.1 & 48.6 & 47.6 & 46.3 & 44.5 & 41.4 & 29.9 \\
\hline A2. Primary balance is unchanged from 2002 & & & 52.0 & 51.5 & 50.9 & 50.0 & 48.4 & 44.7 & 34.8 \\
\hline \multicolumn{10}{|l|}{ Bounds Test } \\
\hline B1. Real GDP growth is at baseline minus two standard deviations in 2003-2004 & & & 52.1 & 51.6 & 51.7 & 51.7 & 50.6 & 48.3 & 44.5 \\
\hline B2. Primary balance is at baseline minus two standard deviations in $2003-2004$ & & & 54.2 & 55.1 & 54.2 & 53.7 & 52.5 & 50.0 & 44.9 \\
\hline B3. Combination of 2-3 using one standard deviation shocks & & & 53.0 & 53.1 & 52.5 & 52.1 & 50.7 & 47.8 & 42.3 \\
\hline B4. Long-run real GDP growth is at baseline minus two standard deviations & & & 51.4 & 50.1 & 50.2 & 50.1 & 49.1 & 46.9 & 43.9 \\
\hline B5. One time 30 percent real depreciation in 2003 & & & 71.6 & 71.3 & 71.2 & 71.5 & 70.4 & 67.6 & 62.7 \\
\hline B6. 10 percent of GDP increase in other debt-creating flows in 2003 & & & 58.2 & 55.3 & 54.6 & 54.3 & 53.2 & 50.8 & 45.6 \\
\hline B7. A permanent, two standard deviation negative shock to the major commodity price in 2003 & & & 51.3 & 49.9 & 49.8 & 49.5 & 48.2 & 45.2 & 40.1 \\
\hline
\end{tabular}

Sources: Country authorities; and Fund staff estimates and projections.

1/ Foreign-currency denominated debt is equal to the total external debt in Table 2 of this Annex. Besides central government debt, government guaranteed debt and the CBK debt to the IMF are included.

2/ Gross financing need is defined as the primary deficit plus debt service.

3/ Debt service is defined as the sum of interest, amortization of medium and long-term debt, and the stock of short-debt at the end of the previous period.

4/ Historical averages are calculated based on numbers for 1998-2002 because data for some of the key variables were not available prior to that period. The historical average for the real GDP growth is 1.0 percent (compared with 3.1 percent in the basel 
Table 2. Kenya: Sensitivity Analyses for Key Indicators of Public and Publicly Guaranteed External Debt, 2003-23 (In percent)

\begin{tabular}{|c|c|c|c|c|c|c|c|c|}
\hline & \multirow{2}{*}{$\begin{array}{r}\text { Estimate } \\
2003\end{array}$} & \multicolumn{7}{|c|}{ Projections } \\
\hline & & 2004 & 2005 & 2006 & 2007 & 2008 & 2013 & 2023 \\
\hline \multicolumn{9}{|c|}{ NPV of debt-to-exports ratio } \\
\hline Baseline & 111 & 109 & 105 & 103 & 100 & 94 & 85 & 91 \\
\hline \multicolumn{9}{|l|}{ A. Alternative Scenarios } \\
\hline A1. Key variables at their historical averages in 2004-23 1/ & 111 & 102 & 85 & 71 & 60 & 54 & 52 & 114 \\
\hline A2. New public sector loans on less favorable terms in 2004-23 2/ & 111 & 112 & 112 & 114 & 113 & 110 & 114 & 157 \\
\hline B1. Real GDP growth at historical average minus one standard deviation in 2004-05 & 111 & 109 & 105 & 103 & 100 & 94 & 85 & 91 \\
\hline B2. Export value growth at historical average minus one standard deviation in 2004-05 3/ & 111 & 125 & 146 & 144 & 138 & 131 & 114 & 110 \\
\hline B3. US dollar GDP deflator at historical average minus one standard deviation in 2004-05 & 111 & 109 & 105 & 103 & 100 & 94 & 85 & 91 \\
\hline B4. Net non-debt creating flows at historical average minus one standard deviation in 2004-05 4/ & 111 & 132 & 150 & 148 & 142 & 135 & 113 & 98 \\
\hline B5. Combination of B1-B4 using one-half standard deviation shocks & 111 & 139 & 173 & 170 & 164 & 155 & 129 & 111 \\
\hline B6. One-time 30 percent nominal depreciation relative to the baseline in $20045 /$ & 111 & 109 & 105 & 103 & 100 & 94 & 85 & 91 \\
\hline
\end{tabular}

\section{Debt service ratio}

Baseline

A. Alternative Scenarios

A1. Key variables at their historical averages in 2004-23 1/

A2. New public sector loans on less favorable terms in 2004-23 2/

\section{B. Bound Tests}

B1. Real GDP growth at historical average minus one standard deviation in 2004-05

B2. Export value growth at historical average minus one standard deviation in 2004-05 3/

B3. US dollar GDP deflator at historical average minus one standard deviation in 2004-05

B4. Net non-debt creating flows at historical average minus one standard deviation in 2004-05 4/

B5. Combination of B1-B4 using one-half standard deviation shocks

B6. One-time 30 percent nominal depreciation relative to the baseline in 2004 5/

Memorandum item:

Grant element assumed on residual financing (i.e., financing required above baseline) 6/
12

$\begin{array}{rrrrrrrr}12 & 11 & 11 & 11 & 11 & 11 & 7 & 11 \\ 12 & 11 & 8 & 8 & 9 & 8 & 10 & 15\end{array}$

12

11

$12 \quad 11$

$12 \quad 11$

$12 \quad 11$

$37 \quad 37$

$\begin{array}{llllll}11 & 11 & 11 & 11 & 11 & 11 \\ 13 & 14 & 14 & 14 & 14 & 13 \\ 11 & 11 & 11 & 11 & 11 & 11 \\ 12 & 13 & 13 & 13 & 14 & 12 \\ 13 & 15 & 15 & 15 & 16 & 13 \\ 11 & 11 & 11 & 11 & 11 & 11 \\ & & & & & \end{array}$

Source: Staff projections and simulations.

1/ Variables include real GDP growth, growth of GDP deflator (in U.S. dollar terms), non-interest current account in percent of GDP, and non-debt creating flows.

2/ Assumes that the interest rate on new borrowing is by 2 percentage points higher than in the baseline., while grace and maturity periods are the same as in the baseline.

3/ Exports values are assumed to remain permanently at the lower level, but the current account as a share of GDP is assumed to return to its baseline level after the shock (implicitly assuming an offsetting adjustment in import levels). A three-year historical average and standard deviation is used for the export stress test.

4/ Includes official and private transfers and FDI.

5/ Depreciation is defined as percentage decline in dollar/local currency rate, such that it never exceeds 100 percent.

6/ Applies to all stress scenarios except for A2 (less favorable financing) in which the terms on all new financing are as specified in footnote 2. 
Figure 1 Kenya: Net Present Value of Total Government Debt

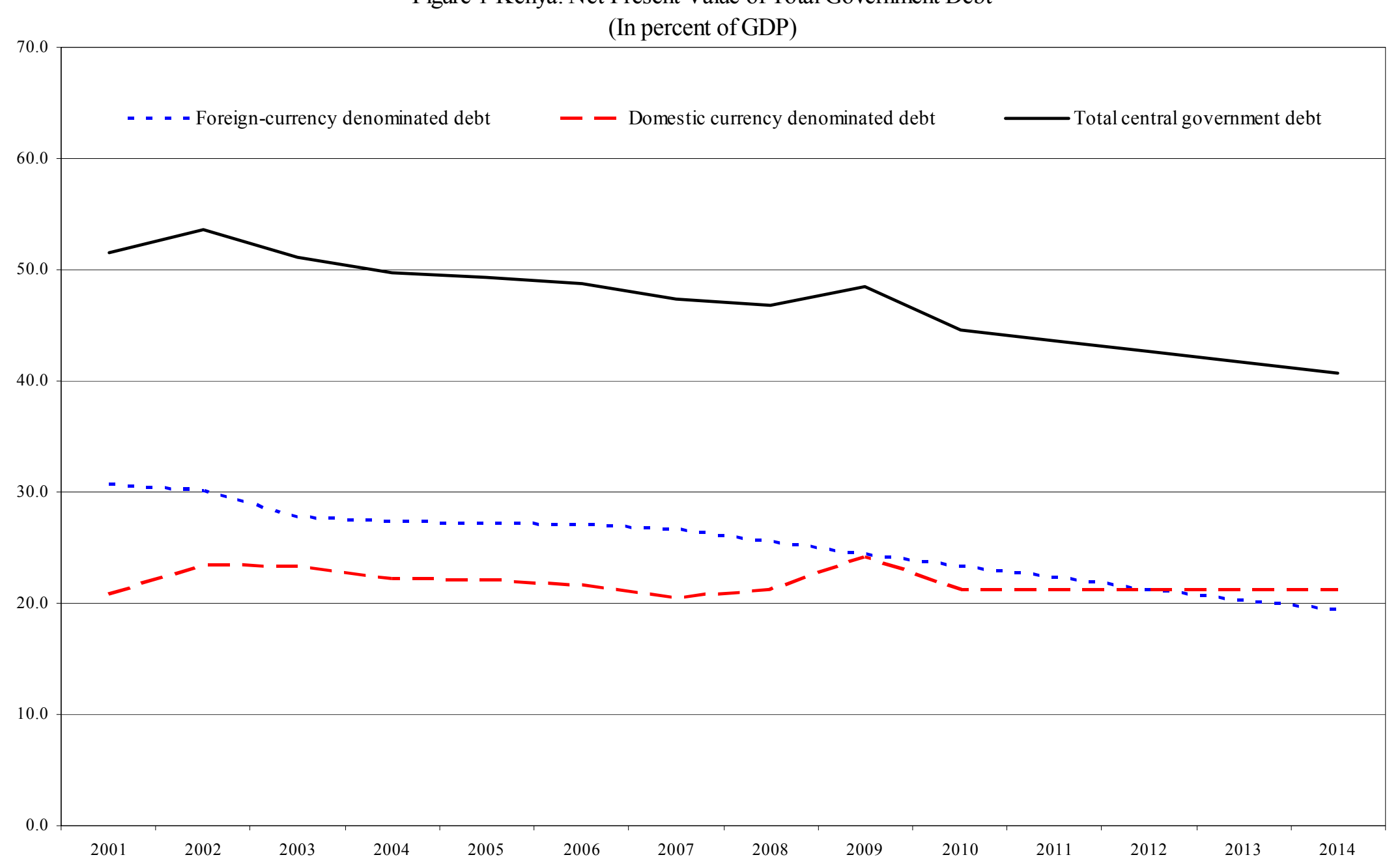

Sources: Kenyan authorities; and staff calculations. 
Figure 2. Kenya: Indicators of Public and Publicly Guaranteed External Debt Under Alternative Scenarios, 2003-2023

(In percent)

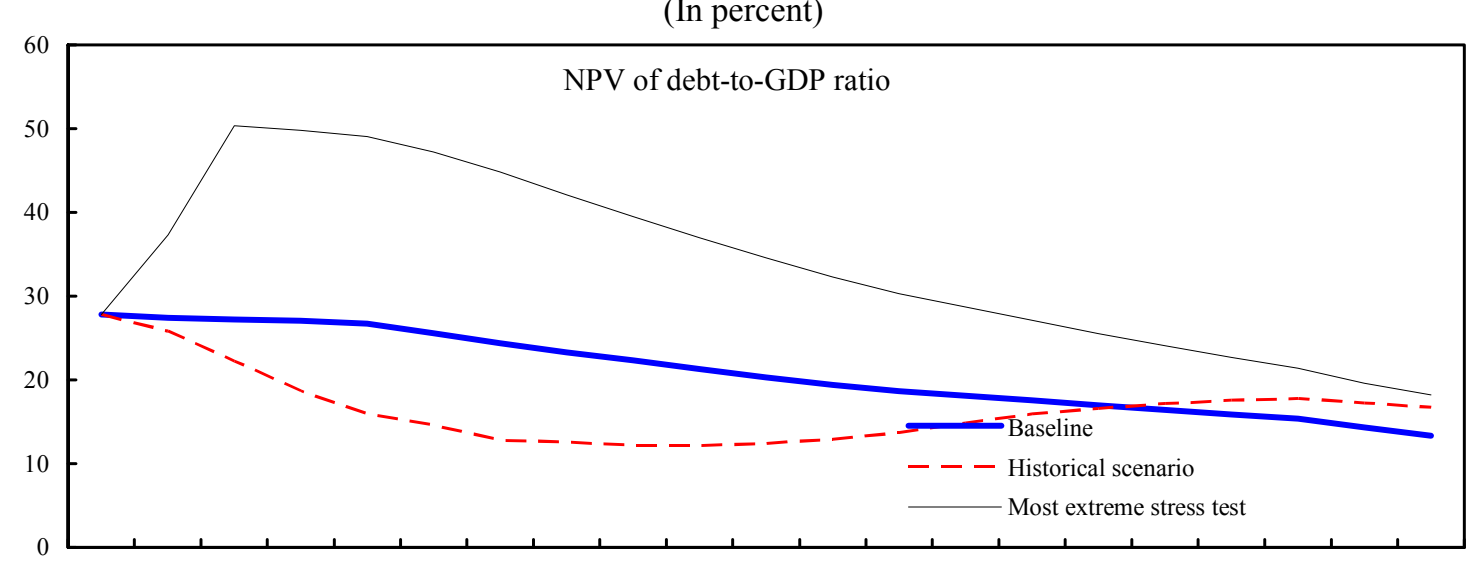

200320042005200620072008200920102011201220132014201520162017201820192020202120222023

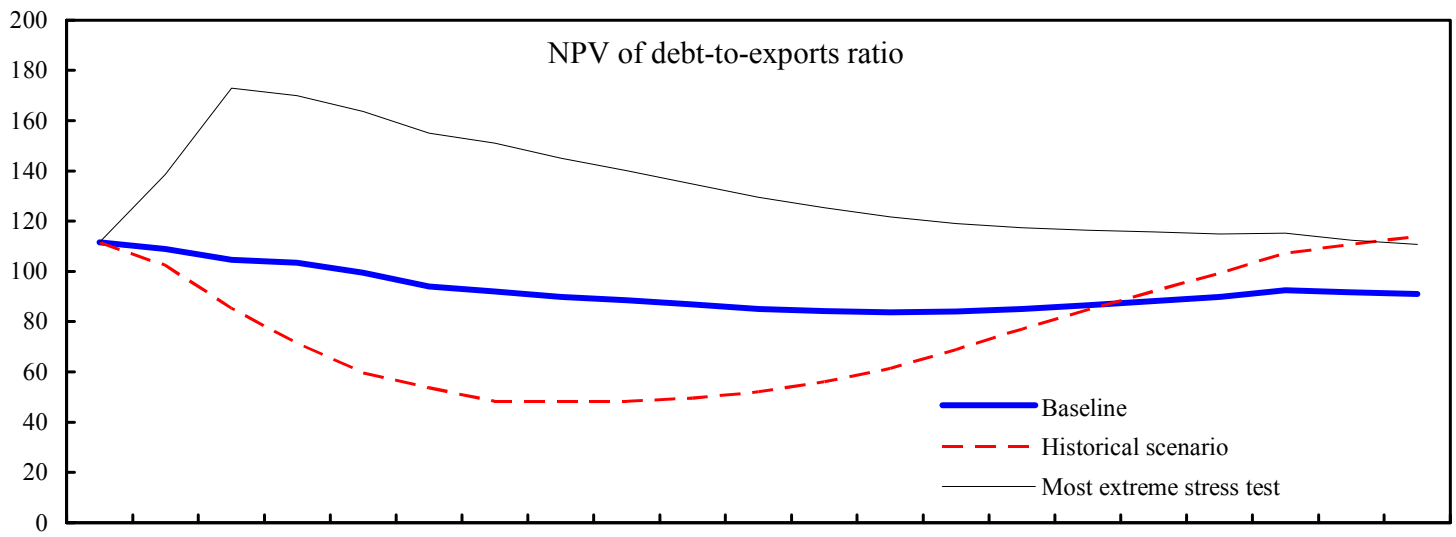

200320042005200620072008200920102011201220132014201520162017201820192020202120222023

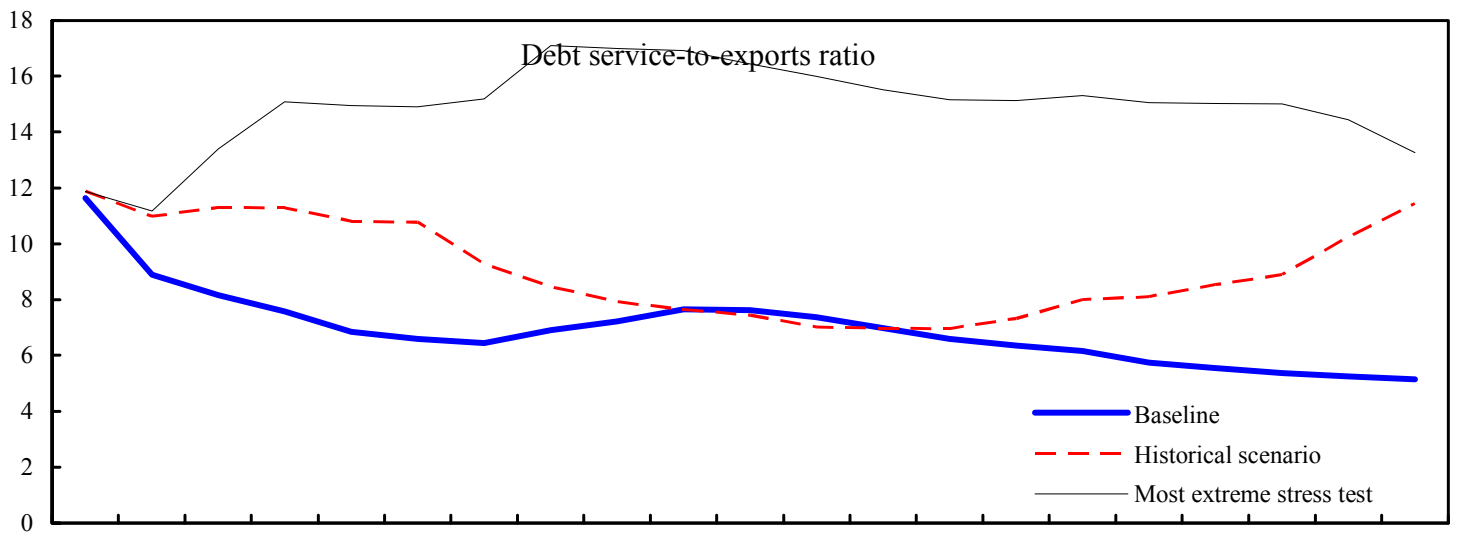

$2003200420052006200720082009201020112012201320142015 \quad 20162017201820192020202120222023$

Source: Staff projections and simulations. 
Kenya: Millennium Development Goals, 1990-2003

\begin{tabular}{|c|c|c|c|c|c|c|c|c|}
\hline & 1990 & 1996 & 1997 & 1998 & 1999 & 2000 & 2001 & 2002 \\
\hline \multicolumn{9}{|l|}{$\begin{array}{l}\text { Goal 1. Eradicate extreme poverty and hunger } \\
\text { Target 1: Halve, between } 1990 \text { and } 2015 \text {, the proportion of people whose } \\
\text { income is less than one dollar a day }\end{array}$} \\
\hline Poverty head count (\%) & 49.0 & .. & 52.0 &.. & .. & .. & 55.0 & .. \\
\hline Population below $\$ 1$ a day $(\%)$ & .. & .. & 23.0 & .. & .. & .. & .. & .. \\
\hline Poverty gap at $\$ 1$ a day $(\%)$ & .. & .. & 6.0 & .. & .. & .. & .. & .. \\
\hline Income share held by lowest $20 \%$ & .. & .. & 5.6 & .. & .. & .. & .. & .. \\
\hline \multicolumn{9}{|l|}{$\begin{array}{l}\text { Target 2: Halve, between } 1990 \text { and 2015, the proportion of people who } \\
\text { suffer from hunger }\end{array}$} \\
\hline Malnutrition prevalence, weight for age ( $\%$ of children under 5 ) & .. & .. & .. & 22.1 & .. & .. & .. & .. \\
\hline Nutrition, undernourished as percentage of total population & 47.0 & .. & 40.0 &.. & .. & .. & 44.0 & .. \\
\hline \multicolumn{9}{|l|}{$\begin{array}{l}\text { Goal 2: Achieve universal primary education } \\
\text { Target 3: Ensure that, by } 2015 \text {, children everywhere, boys and girls alike, } \\
\text { will be able to complete a full course of primary schooling }\end{array}$} \\
\hline School enrollment, primary (\% net) & .. & & .. & 65.8 & 64.9 & 68.2 & 69.9 & .. \\
\hline School enrollment, primary (\% gross) & 95.0 & 83.7 & 85.9 & 90.2 & 89.0 & 93.6 & 96.0 &.. \\
\hline Persistence to grade 5, total ( $\%$ of cohort) &.. & & .. & .. & .. & . & .. & 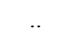 \\
\hline Literacy rate, youth total (\% of people ages $15-24)$ & 89.8 & 93.4 & 93.8 & 94.3 & 94.7 & 95.1 & 95.5 & 95.8 \\
\hline \multicolumn{9}{|l|}{ Goal 3: Promote gender equality and empower women } \\
\hline \multicolumn{9}{|l|}{$\begin{array}{l}\text { Target 4: Eliminate gender disparity in primary and secondary education } \\
\text { preferably by } 2005 \text { and to all levels of education no later than } 2015\end{array}$} \\
\hline Ratio of girls to boys in primary and secondary education (\%) & 94.0 & & .. & 96.8 & 96.3 & 97.2 & 98.0 & \\
\hline Ratio of young literate females to males (\% ages $15-24$ ) & 93.4 & 96.6 & 97.0 & 97.4 & 97.7 & 98.1 & 98.4 & 98.6 \\
\hline $\begin{array}{l}\text { Women wage employment in non-agricultural sector as percentage of total non-agric. } \\
\text { employees }\end{array}$ & 21.4 & 29.4 & 32.2 & 32.1 & 33.8 & 35.6 & 37.8 &.. \\
\hline Proportion of seats held by women in national parliament (\%) & .. & .. & .. &.. &.. & .. &.. &.. \\
\hline \multicolumn{9}{|l|}{$\begin{array}{l}\text { Goal 4: Reduce child mortality } \\
\text { Target 5: Reduce by two-thirds, between } 1990 \text { and 2015, the under-five } \\
\text { mortality rate }\end{array}$} \\
\hline Mortality rate, under- 5 (per 1,000 live births) & 97 & .. & .. & .. & .. & 120 & .. & 122 \\
\hline Mortality rate, infant (per 1,000 live births) & 63 & & .. & & .. & 77 & .. & 78 \\
\hline Immunization, measles ( $\%$ of children under 12 months) & 78.0 & 81.0 & 79.0 & 78.0 & 76.0 & 77.0 & 78.0 & 78.0 \\
\hline \multicolumn{9}{|l|}{$\begin{array}{l}\text { Goal 5: Improve maternal health } \\
\text { Target 6: Reduce by three-quarters, between } 1990 \text { and 2015, the maternal } \\
\text { mortality ratio }\end{array}$} \\
\hline Maternal mortality ratio (modeled estimate, per 100,000 live births) & .. & .. & .. & .. & .. & .. & 590 & .. \\
\hline Births attended by health staff ( $\%$ of total) & 50.0 & .. & .. & 44.3 & .. & .. & 44.0 & .. \\
\hline
\end{tabular}


Kenya: Millennium Development Goals, 1990-2003 (concluded)

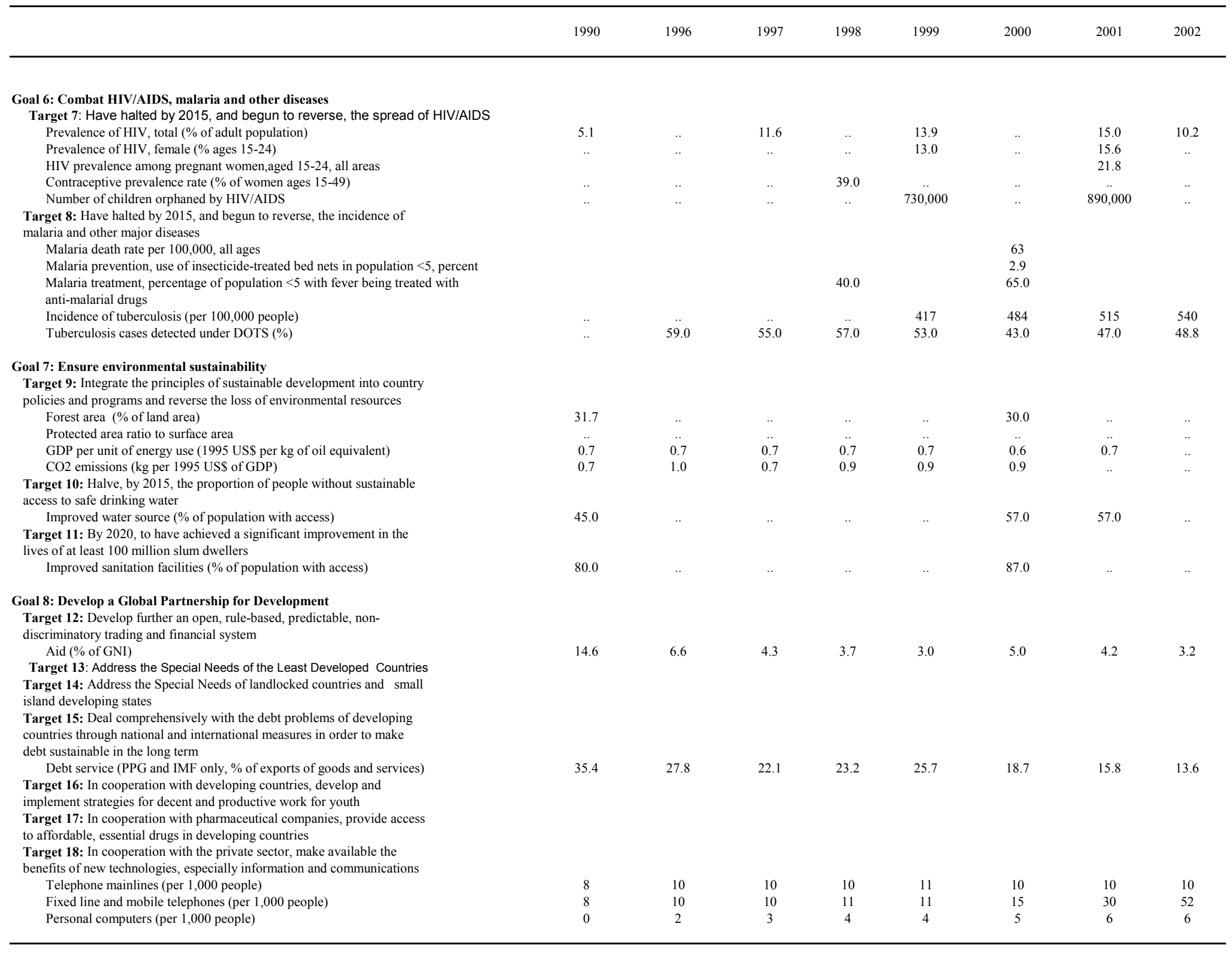


Table 1. Kenya: Selected Social Indicators

\begin{tabular}{|c|c|c|c|c|c|}
\hline \multirow[b]{3}{*}{ Population } & \multicolumn{3}{|c|}{ Kenya, Latest Single Year } & \multirow{2}{*}{$\begin{array}{r}\text { Sub-Saharan } \\
\text { Africa }\end{array}$} & \multirow{2}{*}{$\begin{array}{l}\text { Low-Income } \\
\text { Countries } 1 /\end{array}$} \\
\hline & $1970-75$ & $1980-85$ & $1996-2002$ & & \\
\hline & & & & & \\
\hline Population, total midyear (millions) & 13.7 & 19.9 & 31.4 & 673.9 & 2,506 \\
\hline Population growth & 3.1 & 2.5 & 1.9 & 2.3 & 1.8 \\
\hline Urban population (percent of population) & 12.9 & 19.8 & 35.2 & 33.1 & 30.6 \\
\hline Total fertility rate (births per woman) & 8.0 & 6.9 & 4.2 & 5.1 & 3.5 \\
\hline \multicolumn{6}{|l|}{ Poverty (percent of population) $2 /$} \\
\hline National & $\ldots$ & $\ldots$ & 52 & $\ldots$ & $\ldots$ \\
\hline Urban & $\ldots$ & $\ldots$ & 49 & $\ldots$ & $\ldots$ \\
\hline Rural & $\ldots$ & $\ldots$ & 53 & $\ldots$ & $\ldots$ \\
\hline \multicolumn{6}{|l|}{ Income } \\
\hline GNI per capita, PPP (current international; U.S. dollars) & 340 & 670 & 1010 & 1700 & 2110 \\
\hline GNI per capita, Atlas method (current U.S. dollar) & 250 & 300 & 360 & 450 & 430 \\
\hline \multicolumn{6}{|l|}{ Public expenditure } \\
\hline Health (percent of GDP) & $\ldots$ & $\ldots$ & 8.7 & 6.0 & 4.4 \\
\hline Education (percent of GNI) & 6.3 & 6.4 & 6.5 & 4.1 & 3.3 \\
\hline \multicolumn{6}{|l|}{ Net primary school enrollment rate (percent of age group) } \\
\hline Total & 88 & 91 & 65 & 80 & 80 \\
\hline Male & 93 & 92 & 65 & 86 & 85 \\
\hline Female & 83 & 89 & 65 & 74 & 74 \\
\hline \multicolumn{6}{|l|}{ Access to improved water source (percent of population) } \\
\hline Total & $\ldots$ & 27 & 57 & 58 & 76 \\
\hline Urban & $\ldots$ & 61 & 88 & 83 & 90 \\
\hline Rural & $\ldots$ & 21 & 42 & 46 & 70 \\
\hline \multicolumn{6}{|l|}{ Inmunization rate (percent under 12 months) } \\
\hline DPT & $\ldots$ & 63 & 84 & 59 & 70 \\
\hline Measles & $\ldots$ & 70 & 78 & 57 & 64 \\
\hline Child malnutrition (percent under 5 years) & $\ldots$ & $\ldots$ & 23 & $\ldots$ & $\ldots$ \\
\hline \multicolumn{6}{|l|}{ Life } \\
\hline Life expectancy at birth, total (years) & 52 & 57 & 46 & 47 & 59 \\
\hline Life expectancy at birth, female (years) & 55 & 59 & 46 & 48 & 60 \\
\hline Life expectancy at birth, male (years) & 51 & 55 & 45 & 46 & 58 \\
\hline \multicolumn{6}{|l|}{ Mortality } \\
\hline Under-5 (per 1,000 live births) & 156 & 115 & 122 & 175 & 123 \\
\hline Infant (per 1,000 live births) & 92 & 77 & 78 & 104 & 80 \\
\hline \multicolumn{6}{|l|}{ Adult (15-59) } \\
\hline Male (per 1,000 population) & 467 & 416 & 578 & 519 & 310 \\
\hline Female (per 1,000 population) & 379 & 339 & 529 & 461 & 259 \\
\hline Maternal (per 1,000 population) & $\ldots$ & $\ldots$ & 590 & $\ldots$ & $\ldots$ \\
\hline Births attended by skilled health staff (percent) & $\ldots$ & $\ldots$ & 44 & $\ldots$ & $\ldots$ \\
\hline
\end{tabular}

Source: World Bank, World Development Indicators 2003

1/Low-income countries are those that had a GNI per capita of 755 U.S. dollars or less in 2000.

2/ These data are for 1997. Poverty rates for 1994 are 43.7 percent at the national level, and 29.0 percent and 46.3 percent in urban and rural level. 


\section{Statement by the IMF Staff Representative on Kenya December 20, 2004}

This statement provides information that has become available since the staff report was issued. The thrust of the staff appraisal remains unchanged.

1. We have received confirmation from the Ministry of Finance in Nairobi that the four prior actions for the issuance of the staff report have been met.

- $\quad$ First, the departments responsible for budget monitoring and the medium-term expenditure framework were transferred from the Ministry of Planning and National Development to the Ministry of Finance in September 2004. In addition, new and stronger management teams have been installed in the Debt Management and Fiscal and Monetary Analysis Units of the Ministry of Finance. With these actions, the authorities have fulfilled the prior action relating to the restructuring and strengthening of the capacity of the Ministry of Finance to manage macroeconomic and fiscal policies.

- $\quad$ Second, expenditure releases are now managed in the context of the program's fiscal framework, which limits domestic borrowing in 2004/05 to Ksh 32 billion (2.5 percent of GDP). Domestic borrowing would likely be lower than the agreed target as the increase in revenue collection, at about 25 percent during the first five months of 2004/05, is well above the assumed increase of 7 percent under the program.

- $\quad$ Third, the government has agreed on a system of annual declarations and verifications of the assets of ministers, permanent secretaries, and heads of state corporations.

- $\quad$ Finally, the government completed in November 2004 the Budget Outlook Paper for 2005/06-2007/08.

2. With regard to the Central Bank of Kenya (CBK) (Amendment) and the Banking (Amendment) Bills that are alluded to in both the Financial System Stability Assessment and staff report, Parliament passed the two bills last week. However, while the CBK (Amendment) Bill was passed as presented, the Banking (Amendment) Bill was modified, with the retention of Section 44 of the Act, which requires the prior approval of the Minister of Finance when setting banks' fees and charges.

\section{The main implications of these changes are:}

- $\quad$ Virtually all banking sector regulatory and supervisory authority have been transferred from the Ministry of Finance to CBK; and 
- $\quad$ The provisions of the CBK (Amendment) Act that provided for the control of interest rates have been removed.

4. Presentation to parliament of an amendment to the Banking Act to provide for the transfer of all financial sector regulatory functions from the Ministry of Finance to the CBK was a structural performance criteria under the program for end-March 2004. With regard to Section 44 of the Banking Act, the Minister of Finance has confirmed that the government will continue to desist from applying the regulation. 


\section{INTERNATIONAL MONETARY FUND}

Public Information Notice (PIN) No. 09/75

FOR IMMEDIATE RELEASE

June 12, 2009
International Monetary Fund

$70019^{\text {th }}$ Street, NW

Washington, D. C. 20431 USA

\section{IMF Executive Board Concludes 2004 Article IV Consultation with Kenya}

On December 20, 2004, the Executive Board of the International Monetary Fund (IMF) concluded the Article IV consultation with Kenya. ${ }^{12}$

\section{Background}

During 2003/04, notable progress has been made in reversing the lackluster performance of the previous decade. A moderate rebound in economic growth has begun and the recent sharp growth in domestic debt has been arrested. However, inflationary pressures have increased.

The moderate recovery in economic growth that began in 2003/04 is expected to continue, with the real Gross Domestic Product (GDP) growth projected at 2.7 percent in 2004/05 after an estimated 2.1 percent in the previous year. Both the tourism and construction sectors are projected to register strong growth. Reflecting the adverse effects of the drought on food prices and the recent sharp increase in oil prices, headline consumer price inflation has accelerated sharply in recent months and reached 16.6 percent in October 2004.

Monetary policy has been expansionary since 2003/04 to promote economic growth. This has, however, contributed to the recent rise in inflation, and ex post real interest rates were negative over much of the past year. In response to the significant pick up in

\footnotetext{
${ }^{12}$ Under Article IV of the IMF's Articles of Agreement, the IMF holds bilateral discussions with members, usually every year. A staff team visits the country, collects economic and financial information, and discusses with officials the country's economic developments and policies. On return to headquarters, the staff prepares a report, which forms the basis for discussion by the Executive Board. At the conclusion of the discussion, the Managing Director, as Chairman of the Board, summarizes the views of Executive Directors, and this summary is transmitted to the country's authorities.
} 
both headline and underlying inflation, the Central Bank began to tighten monetary policy in September 2004, and three-month Treasury bill rates have increased from 2.75 percent in September to 7.2 percent in late November 2004.

Recent economic developments may have worsened poverty indicators. With real GDP growth broadly in line with the increase in population and the economy suffering from the effects of major negative shocks - the drought and the oil price increase-the poverty rate may have increased in the recent past. Moreover, progress in poverty alleviation has been hampered by AIDS and delays in initiating major poverty reduction programs.

Overall fiscal performance strengthened during 2003/04 mainly on account of strong revenue collection and the steps taken to control recurrent expenditure. Some capital spending was also curtailed on fears that the resulting large domestic borrowing would raise interest rate pressures. As a result, domestic borrowing was much lower than budgeted and the ratio of domestic debt to GDP fell from 24.3 percent at end-June 2003 to 22.2 percent of GDP at end-June 2004.

The current account balance turned from a surplus of 1.2 percent of GDP in 2002/03 to a deficit of 2.0 percent in 2003/04, reflecting primarily a drop in private savings. Private savings were negatively affected by negative real interest rates, but also by the drought and the increase in oil prices. Export volumes grew by 7 percent in 2003/04, as tea, horticultural, and garment exports performed well. With a strong capital account position, foreign reserves rose by about U.S. $\$ 140$ million, but declined moderately in percent of imports.

Progress in implementing structural reforms has been mixed. Some progress was made in strengthening governance, and there were some delays in implementing reforms in the public expenditure, financial sector, and parastatal areas. New wage-setting mechanisms for public employees have been developed.

\section{Executive Board Assessment}

Executive Directors observed the progress that has been made under the PRGFsupported program, which has contributed to a rebound in economic growth and arrested the rise in domestic debt. However, Directors noted that Kenya faces serious challenges. In particular, growth remains too slow for making inroads into poverty, inflation has picked up, and governance remains a critical concern.

Directors emphasized that Kenya's medium-term economic prospects depended critically on the prompt and effective implementation of broad-based structural reforms as outlined in the authorities' Poverty Reduction Strategy Paper (PRSP). They stressed the importance of promoting strong economic growth and ensuring that its benefits are translated into poverty reduction and improved living standards for the rural population. Implementation of strong policies and a faster pace of reforms would help achieve increased donor budgetary support, and Kenya would be in a position to begin to make progress toward the Millennium Development Goals. 
Directors recognized the efforts that have been made to strengthen governance. They welcomed efforts to build a more robust public financial management system, the establishment of the Kenya Anti-Corruption Commission, and the ongoing strengthening of prosecutorial capacity. Directors observed, however, that important gaps remained in implementing the governance agenda, and they stressed the need for more determined actions. In this connection, Directors emphasized the importance of adequate funding of key anticorruption agencies; full investigation, disclosure, and punishment of those involved in corruption cases; and improved rules for the management of security procurements. There were also calls for continued efforts in the area of asset declarations by senior officials, including ministers, permanent secretaries, and heads of state bodies.

Directors expressed concern with the recent upturn in inflation. They urged the authorities to maintain firm control over monetary aggregates as several factors, such as high oil prices, the uncertain food supply situation, and exchange rate developments point to a risk of persistent price pressures. Progress in containing wage costs would be important for reducing inflation and, in turn, for promoting financial intermediation. Directors agreed that the current managed floating exchange rate system has served Kenya well in responding to external shocks, and should be maintained. A few Directors saw scope for increased flexibility in this regard.

Directors supported the authorities' effort to balance their commitment to fiscal consolidation with the need to support the poverty reduction strategy. They welcomed the lower-than-programmed deficit in 2003/04. However, Directors noted that significant fiscal risks remained, as deficits were projected to remain high and the magnitude of donor support uncertain. Some Directors pointed to the fiscal risks associated with weak public enterprises and the costs of addressing HIVIAIDS. In this connection, Directors welcomed the authorities' efforts to mobilize increased domestic resources through ongoing reforms of tax administration and steps to improve customs services.

Directors also noted that meeting the fiscal objectives will require effective expenditure monitoring and control. They stressed the importance of building a robust public expenditure management (PEM) system. Directors welcomed the authorities' efforts to modernize the PEM system, including strengthening the links between the medium-term expenditure framework and the annual budget system, with the support of the World Bank and the Fund. They urged the authorities to strengthen further existing institutions and procedures, with a view to enhancing the poverty orientation of public outlays. In addition, Directors recommended close monitoring of the debt situation, and advised against contracting any new nonconcessional debt.

Directors underscored the importance of accelerating the public enterprise restructuring and privatization program. They urged the authorities to make a strong effort to secure parliamentary enactment of the Privatization Bill and to follow up quickly with its implementation. In this context, they welcomed the authorities' decision to start, in the near future, a detailed assessment of the financial position of key parastatals. 
Directors were encouraged by the authorities' increased attention to improving competitiveness. They supported the authorities' broad-based strategy that includes streamlining the regulatory framework, upgrading essential infrastructure, reforming the wage-setting system, and liberalizing trade. However, Directors noted the deteriorating trend in total factor productivity in Kenya, and urged stronger efforts to promote privatesector led growth, including through further trade liberalization, regional integration, and improved governance.

Directors welcomed the actions taken by the authorities to strengthen the financial system, including the recent approval of the amendments to the Central Bank of Kenya and Banking Acts, which transfer bank regulatory functions from the Ministry of Finance to the central bank and remove the provisions for official controls of interest rates. Directors looked forward to the removal of government control over bank charges and fees. Directors observed, however, that the financial system remains fragile. While banking soundness indicators have generally improved, the large nonperforming loans (NPLs) remain a problem. They therefore urged the authorities to address the problem of distressed banks, particularly public banks, which held most of the large NPLs, and to move forward promptly with bank privatizations. Directors pointed to the need to move forward with a strengthening of the framework for Anti-Money Laundering/Combating the Financing of Terrorism (AML/CFT).

Directors took note of the challenges posed by capacity constraints for policy implementation. They observed that shortages of skilled personnel and weaknesses in public administration, particularly in key ministries, constrained the implementation of essential poverty programs and the absorption of donor-project support. Directors, therefore, welcomed the restructuring efforts that have been initiated to rebuild capacity in the Ministry of Finance and key line ministries. They also welcomed ongoing efforts to restructure the civil service and develop new wage setting mechanisms for public employees, noting that the realization of the poverty reduction objective depended upon improved delivery of public services.

Directors generally welcomed the steps underway to improve the quality of economic and social statistics. They noted that the government has released a revised set of national accounts data, was taking steps to improve the monitoring of fiscal data and intended to develop a framework for the systematic monitoring and evaluation of productivity changes. However, the quality of statistics for surveillance and program monitoring remains weak, and Directors urged further efforts to improve statistics, with the support of the African Technical Assistance Center (AFRITAC), as appropriate.

Public Information Notices (PINs) form part of the IMF's efforts to promote transparency of the IMF's views and analysis of economic developments and policies. With the consent of the country (or countries) concerned, PINs are issued after Executive Board discussions of Article IV consultations with member countries, of its surveillance of developments at the regional level, of post-program monitoring, and of ex post assessments of member countries with longer-term program engagements. PINs are also issued after Executive Board discussions of general policy matters, unless otherwise decided by the Executive Board in a particular case. 
Kenya: Selected Economic Indicators, 2000/01-2003/04 1/

2000/01 2001/022002/032003/04

Prelim.

\section{Output and prices}

GDP volume (factor cost)

Consumer price index (annual average)

Nominal effective exchange rate (- depreciation; end of period)

Real effective exchange rate (- depreciation; end of period)

\section{Money and credit}

M3X (M3 plus foreign currency deposits, end of period)

Reserve money (end of period)

Interest Rate (90-day Treasury Bill, period average )

\section{Central government budget}

Total revenue

(Annual percent change, unless otherwise indicated)

Total expenditure and net lending

Overall balance (commitment basis) excluding grants

Net domestic borrowing

Total donor support (grants \& loans)

$\begin{array}{llll}0.5 & 1.2 & 1.5 & 2.1 \\ 10.0 & 2.3 & 6.6 & 8.2 \\ 8.3 & -5.9 & -5.2 & -11.1 \\ 10.2 & -4.7 & 4.7 & -8.2\end{array}$

$\begin{array}{llll}2.1 & 6.7 & 10.9 & 12.9 \\ -8.5 & 10.9 & 11.2 & 5.5 \\ 12.1 & 10.9 & 7.2 & 1.6\end{array}$

(In percent of GDP)

\section{Balance of payments}

Exports goods, f.o.b. (in million of U.S. dollars)

Imports goods, f.o.b. (in million of U.S. dollars)

Current external balance, excluding official transfers

Gross international reserve coverage

in months of next year imports (end of period)

$\begin{array}{llll}23.0 & 21.5 & 20.5 & 21.7 \\ 27.8 & 24.9 & 25.9 & 23.5 \\ -4.8 & -3.4 & -5.4 & -1.7 \\ 0.1 & 4.3 & 4.6 & 0.8 \\ 4.5 & 1.9 & 2.2 & 2.4\end{array}$

Public Debt

NPV of central government debt (end of period)

$\begin{array}{llll}1,870.0 & 1,962.0 & 2,322.0 & 2,491.0 \\ 3,310.0 & 2,823.0 & 3,215.0 & 3,870.0 \\ -3.7 & -0.8 & 1.2 & -2.0 \\ & & & \\ 3.5 & 3.3 & 3.3 & 3.0\end{array}$

Domestic debt, net

$\begin{array}{llll}54.0 & 53.4 & 51.9 & 49.6\end{array}$

External debt

$\begin{array}{llll}19.6 & 22.0 & 24.3 & 22.2\end{array}$

$\begin{array}{llll}34.4 & 31.4 & 27.6 & 27.4\end{array}$

Sources: Kenyan authorities; staff estimates and projections.

$1 /$ Fiscal year period is from July 1 to June 30 
Press Release No. 04/270

FOR IMMEDIATE RELEASE

December 20, 2004

Corrected: 05/12/05
International Monetary Fund

Washington, D.C. 20431 USA

\section{IMF Executive Board Completes First Review Under Kenya's PRGF Arrangement and Approves US\$76.9 Million Disbursement Kenya}

The Executive Board of the International Monetary Fund (IMF) today completed the first review of Kenya's economic performance under a three-year Poverty Reduction and Growth Facility (PRGF) arrangement, which was approved on November 21, 2003 (see Press Release No. 03/201). The Board also approved waivers for the nonobservance of continuous and structural performance criteria.

The Executive Board approved augmented access under the PRGF arrangement by SDR 50 million (about US\$ 76.9 million), which will bring the total amount drawn under the arrangement to about SDR 75 million (about US\$115.4 million).

Following the Executive Board discussion, Mr. Rodrigo de Rato, Managing Director and Chair, said:

"Progress has been made in implementing policy reforms under the three-year program supported by the Fund. A rebound in economic growth has begun, the recent sharp increase in domestic debt has been arrested, and implementation of key structural reforms is underway. In addition, the authorities have begun to address capacity constraints and to strengthen key policy implementing institutions.

"The main challenge is to accelerate broad-based growth to help reduce poverty and improve the delivery of essential social services, while also securing fiscal consolidation and low inflation. In the near term this will require prudent fiscal management and firm control over monetary aggregates to bring down inflation. Over the medium term, tight fiscal policy will need to be supported by a broadening and acceleration of structural reforms to enhance economic efficiency, expand the role of the private sector in the economy, and promote increased donor support. 
"Consistent with these objectives, the authorities' program for 2004/05 emphasizes policies to bring down inflation and strengthen budget management. It focuses on measures that aim at improving the efficiency of public expenditure and increasing revenue collection. The program also envisages significant advances in the governance and anti-corruption area, where some progress has been made, but much remains to be done. To strengthen Kenya's external competitiveness, a multifaceted strategy is being implemented that includes streamlining the regulatory framework, reforming the wage-setting system and liberalizing trade. Moreover, a strengthening of the financial system is envisaged through a variety of reforms, including actions to address the problems of distressed state-owned banks, reduce the large number of nonperforming loans, and promote privatization.

"The government's reform program is well tailored to meet the pressing economic challenges facing Kenya. Strengthened implementation of the reform agenda, particularly in the area of governance, is essential for achieving enhanced donor support, sustainable growth, and poverty reduction," Mr. de Rato said.

The PRGF is the IMF's concessional facility for low-income countries. PRGF-supported programs are based on country-owned poverty reduction strategies adopted in a participatory process involving civil society and development partners and articulated in a Poverty Reduction Strategy Paper (PRSP). This is intended to ensure that PRGF-supported programs are consistent with a comprehensive framework for macroeconomic, structural and social policies to foster growth and reduce poverty. PRGF loans carry an annual interest rate of 0.5 percent and are repayable over 10 years with a $5 \frac{1}{2}$ year grace period on principal payment. 


\section{Statement by Peter Ngumbullu, Executive Director for Kenya and Peter Gakunu, Alternate Executive Director December 20, 2004}

\section{Introduction and Background}

1. The Kenyan authorities are grateful to staff for the high quality set of papers and for the candid exchange of views during the recent Article IV consultation discussions and first review under the PRGF arrangement. The authorities agree with the thrust of the staff assessment, and view the reports as fair and balanced in addressing economic development issues and the challenges facing the country. They request the support of the Executive Board for the completion of the First Review under the Poverty Reduction and Growth Facility, Augmentation of Access, Rephasing of the Arrangement and Waiver of Performance Criteria.

2. Kenya has made significant progress in its second year of implementing reforms under the PRGF arrangement to consolidate its economic gains, as well as develop a realistic and ambitious medium term framework to guide economic policy-making. The authorities are actively pursuing prudent macroeconomic policy measures, including implementation of wide-ranging reforms. Expenditures have been prioritized in the context of the Economic Recovery Strategy (ERS) objectives with a gradual reorientation towards pro-poor expenditure and investments. Better coordination procedures have been put in place with donors, to ensure efficient fiscal management and mobilization of increased resources for poverty reduction. There is strong political will by the authorities to pursue policies of enhancing the investment climate, strengthening governance institutions, as well as deepening of the financial sector. The authorities have succeeded in solidifying the consensus required for successful enactment of a new constitution.

3. Notwithstanding these positive developments, the country faces enormous developmental challenges in a number of areas, including achieving fiscal sustainability, improving capacities and budgetary resources to enhance effectiveness of key governance institutions, reforming the labor market, upgrading infrastructure, and deepening the structural reform agenda. The authorities are aware of these, and have incorporated the necessary corrective measures under its Economic Recovery Strategy (ERS). They would like to use this opportunity to reiterate their continued commitment to sound macroeconomic management and the reform agenda.

\section{Recent Economic Developments and Prospects}

4. Despite high oil prices and a severe drought, the Kenyan economy is estimated to have grown by 2.1 percent in 2004, reflecting a recovery of the tertiary sectors. Looking ahead, real GDP is projected at 2.7 percent in 2004/05, lower than the 3.1 percent expected in the original program, reflecting in part the negative effects of high oil prices and depressed prices for some traditional exports, as well as the impact of drought on agricultural production. The medium-term macroeconomic prospects depend mostly on the pace of reforms, fiscal consolidation, and resumption of strong donor support. This notwithstanding, our authorities are concerned that although the 
medium-term growth rates appear reasonable when seen against Kenya's recent performance, they fall far short of the ERS target of 6 percent, which implies a significant delay in the achievement of the MDGs. The authorities have consequently strengthened domestic revenue effort, restructured expenditure and have accelerated management reforms, among other initiatives.

\section{Structural Reforms}

5. Since December 2003, the authorities have implemented an ambitious and multi-faceted program of reforms aimed at improving governance, including passing key anti-corruption and public ethics legislation, strengthening the judiciary and the public audit system, as well as implementation of the United Nations Convention against Corruption and Ratification of the African Union Convention on Corruption. Other reforms include: measures to enhance the effectiveness of key anti-corruption investigative agencies, such as the swearing in of senior personnel in the Kenya Anti-Corruption Commission in September 2004, and the hiring of additional personnel targeted for completion by July 2005; and the implementation of a Public Officer Ethics Act under which all public officials, including the executive and legislative arms of government and the military have completed the first round of declaration of their assets and those of their families and are in the process of completing the second round.

6. Additional measures that have been undertaken by the authorities include: strengthening of the judiciary under a newly appointed Chief Justice; completion of pro-poor expenditures for both recurrent and development expenditures; preparation of comprehensive program of public finance reforms; strengthening of Central Bank of Kenya (CBK) operations; deepening transparency in the conduct of public operations; improving the procurement system; addressing the outcomes of recent initiatives to address past economic crimes; strengthening of public agencies, through implementation of the recommendation of the task force on reform of the police beginning December 2004; enhancing public interaction with the government on governance, by establishing a Public Complaints Unit (PCU) to serve as a central referral and monitoring and evaluation unit under the aegis of the Department of Governance and Ethics, in conjunction with the Ministry of Justice and Constitutional Affairs; and initiation of comprehensive land reforms including forming various committees and a commission which has currently completed its initial work and its report published.

7. The slow progress in moving toward a comprehensive medium-term expenditure framework, consistent with PRSP priorities, as well as in instituting a more robust expenditure management system, is largely attributable to organizational and capacity constraints at the Ministry of Finance, that are now being addressed. Financial sector and parastatal reforms suffered from capacity constraint, but also lack of consensus on the objectives and modalities for conducting the reforms that have delayed the enactment of the Privatization Bill. New wage-setting mechanisms for public employees have now been developed. Significant progress has been made in building a robust governance architecture and in enforcing elements of the anti-corruption regulations. The authorities have taken decisive steps to strengthen the prosecution branch. The budget for Kenya Anti-Corruption Commission (KACC) has been increased significantly. Legal investigations on the 
Anglo-Leasing scandal are proceeding well. All the senior officials involved in the scandal have been suspended and the authorities have confirmed that once the investigations are completed prosecution of those involved will commence. On the Kenya Urban Transport Infrastructure Projects (KUTIB), the culprits are in the courts after being dismissed from their jobs. Furthermore, an external forensic audit, supported by the World Bank was completed two months ago, the finding presented to the Minister of Finance, and the Government is taking action based on the results of the audit. The Minister has approached the World Bank to conduct further forensic audits on a number of projects with a view to taking appropriate action.

\section{Fiscal Policy}

8. The monetary policy pursued by the authorities aims at supporting non-inflationary economic recovery. However, following the reduction in the legal reserve requirement in July 2003, the reserve money multiplier rose putting downward pressure on yields on money market instruments. In response to the decline in interest rates, bank credit to the private sector grew substantially resulting in inflationary pressure. The inflation objective is to be achieved through reserve money targeting, with broad money as the intermediate target and open market operations as the main instruments. In this connection, the recently enhanced open market operations and treasury bill auctions have resulted in increased real interest rates that should help stem the threat to resurgence in inflation. The $\mathrm{CBK}$ will continue to use a broad set of indicators to monitor future inflationary pressures and to gauge the appropriateness of the monetary policy stance. The authorities will continue to implement a flexible exchange rate system, which has served the economy well in absorbing fluctuations in external conditions. The CBK's intervention in the foreign exchange market will be limited to meeting the net foreign assets target under the program and to smoothen the disruptive short-term fluctuations.

\section{Fiscal Policy}

9. The overall fiscal performance was better than anticipated by the program with central government fiscal imbalances being much lower than expected, reflecting the strong commitment of the authorities to stamp out corruption and the improved capacity and governance of the institutions involved in revenue collection, as well as the steps taken by the authorities to strengthen the management of recurrent expenditure. The authorities' fiscal policy stance aims at continuing support of macroeconomic stability and addressing fiscal sustainability among other steps. This would be achieved by strengthening revenue performance, lowering the wage bill as a proportion of GDP, including establishing a new wage setting mechanism for public employees, further tackling contingent fiscal liabilities, strengthening tax administration, implementing public expenditure management (PEM) reforms, continuing civil service rationalization, and increasing the poverty reduction spending. Another key component of the fiscal programme is domestic debt reduction aimed at debt sustainability in which the government does not crowd-out the private sector without compromising the provision of increased resources for priority poverty reduction spending. 
A revision to the budget announced on June 12, 2004, will be presented to Parliament during the first quarter of 2005. Pending this revision, the new fiscal framework will be implemented through quarterly releases of expenditures. The revised budget assumes that domestic borrowing would amount to the equivalent of 2.5 percent of GDP. The fiscal framework envisages a marked decline in both the overall and primary deficits relative to ERS targets, wholly because of the significant shortfall of donor budgetary support. For the same reason and the need to preserve some essential social and economic programs, domestic borrowing would exceed ERS expectations. Some crowding out of private activity is expected, as the public sector would absorb a sizable proportion of projected financial savings. Reflecting the projected domestic borrowing, total domestic debt would remain broadly unchanged at 22 percent of GDP compared with the original program target of 24.7 percent of GDP. The authorities aim at reversing the recent trend towards increased current spending by raising capital outlays from 2.7 percent of GDP in 2003/04 to 4.4 percent of GDP in 2004/05 with allocations for roads, health and agriculture accounting for the bulk of the increase.

10. The authorities have in their unwavering fight against corruption, requested for a comprehensive audit of external commercial contracts which has necessitated a relatively small accumulation of arrears. In recognition of this, the authorities have established an escrow account for settling fully all legitimate debt once the audit is completed and they are looking for ways of managing the near-term temporary increase in domestic borrowing and domestic debt, which will be a major challenge for them. In this connection, they plan to strengthen the revenue performance by maintaining revenue as a proportion of GDP of at least 21.5 percent, broadening the tax base by removing most exemptions, taxing a larger share of informal transactions and simplifying and rationalizing the tax system. Looking ahead, the wide-range of measures taken by the authorities to strengthen revenue collection will be supported by controlling and prioritizing expenditure programs, cutting non-essential outlays as well as the resumption of strong donor support.

\section{Financial Sector Issues}

11. The Kenyan authorities are actively pursuing reforms of the financial system to enhance financial intermediation. They have already started to implement the reforms proposed by the recent FSAP recommendations drawing among others on support by the World Bank in preparation for a Financial Sector Adjustment Credit (FSAC). The measures that are being implemented are designed to strengthening the financial system, including passing of amendments to the CBK and Banking Acts by Parliament, new regulations, and tightening loan provisioning and classification, which will be introduced in 2005. Recently there has been a notable improvement in banking sector performance indicators, including fall in the non-performing loans. Central features of the authorities financial sector development plan include focusing on promoting financial intermediation in rural areas, as well as increasing the access of small and medium -sized firms to formal sector financial resources. In this connection, they will work hard to broaden the scope and outreach of micro finance institutions and for a more coherent and targeted policy for promoting development finance institutions. 


\section{Trade Reforms and Regional Integration Efforts}

12. The authorities plan to address international competitiveness through reforms in the utility and telecommunications sectors, in external trade and the labor market, as well as upgrading of infrastructure. Kenya is expected to form a customs union with Tanzania and Uganda in 2005, in the context of the East African Community (EAC). Kenya is also a member of the Common Market for Eastern and Southern Africa (COMESA), which aims at the establishment of a customs union through the removal of all trade barriers, and a common external tariff and rules of origin. The IMF and the Bank are providing assistance with reform of the tariff regime, and the Bank is providing technical advice on the regional integration issues.

\section{Poverty Reduction Strategy Process}

13. The development strategy as outlined in the ERS is part of a broader economic agenda that is supported by donors and development agencies, including the World Bank. The programme contains a package of policy measures aimed at addressing the identified economic problems of low growth, low savings and investment, poor public service, large domestic debt, distortions in the financial system, unemployment, and poverty. Embedded in the programme are core poverty expenditures, which are derived from specific development targets that include, among other measures, improving access to primary education, health care and providing other safety nets. Since most of the poor live in rural areas, the priority is to reform the agricultural sector and encourage growth of medium and small-scale enterprises, while plans are underway to upgrade micro finance institutions. In addition, there are measures to tackle the high prevalence of HIV/AIDS in rural areas, including the rehabilitation of rural health facilities to positively affect agricultural productivity and rural incomes.

14. Our authorities are aware of the challenges they face in implementing the programme, and they have noted staff's comments and recommendations for addressing them. In this regard, in addition to the reforms already undertaken and those that are ongoing, they have identified and incorporated into the ERS programme, other policy measures that will be needed to pre-empt many of the risks and vulnerabilities, including initiation of a new anti-money laundering legislation that will reflect all relevant recommendations from the Bank-Fund FSAP assessment report for Kenya; and improving the quality and timeliness of data in the areas of the budget, foreign trade and national accounts. With respect to the latter, a data ROSC mission has been invited and will be undertaken in early 2005.

\section{Need for Donor Support}

15. Medium-term projections indicate that there will be external account gaps in implementing the new programme arising from an envisaged pickup in government outlays on social and economic infrastructure and other public investment programmes. These outlays will put substantial pressure on the external current account. The deficit on the external current account is estimated to widen to 4.9 per cent of GDP in 2003/04, with the prospects of rising further to 11.0 per cent of 
GDP by 2005/006. Consequently, the programme will require a marked increase in donor assistance from 6.5 per cent of GDP to 9.9 per cent in 2005/06 to support its implementation. Total financing needs are projected to average about $\$ 1.3$ billion per year through 2005/06. Program support and Paris Club rescheduling are expected to fill the financing gap during the period. The authorities have indicated their intention to seek a rescheduling from the Paris Club and are currently discussing with their key creditors and donors on possible Paris Club rescheduling terms.

\section{Conclusion}

16. In conclusion, our Kenyan authorities wish to reiterate their strong political will and commitment to revive the Kenyan economy and put it on the path of sustainable and job creating growth and poverty reduction. They have made the fight against corruption a priority, and have achieved significant progress in the implementation of the reform agenda. Fiscal performance was better than anticipated in the program and monetary policy has been tightened appropriately to support the objective of price stability, necessary for long-run growth. Overall, the economy is in a much better state than when the new coalition government took office two years ago. However, in consolidating the gains of macro-economic stabilization, the authorities hope that they can continue to count on the sustained understanding and support of the Fund and the rest of the international community in the form of timely and adequate financial support, advice and technical assistance in the relevant areas to achieve these objectives. They are taking measures to implement reforms under the PRGF arrangement to consolidate the economic gains achieved in the reform agenda to sustain poverty reduction, economic growth, meet the MDGs, and improve the living standards of the Kenyan people. 